\title{
Highlighting Diffusional Coupling Effects in Ternary Liquid Extraction and Comparisons with Distillation
}

\author{
Rajamani Krishna \\ Van 't Hoff Institute for Molecular Sciences, University of Amsterdam, Science Park 904, \\ 1098 XH Amsterdam, The Netherlands
}

email: r.krishna@contact.uva.nl 


\section{Table of Contents}

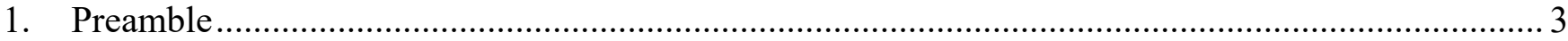

2. Transient equilibration in heptane(1)/toluene(2)/sulpholane(3) mixtures ......................................... 3

3. Transient equilibration in NMP(1)/propylbenzene(2)/tetradecane(3) ............................................. 12

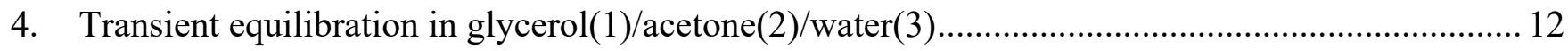

5. Transient equilibration in water(1)/acetone(1)/ethyl-acetate(3) ...................................................... 13

6. Transient equilibration in water(1)/caprolactam(2)/toluene(3)........................................................ 14

7. Transient equilibration in water(1)/acetic acid(2)/isophorone(3) ................................................. 14

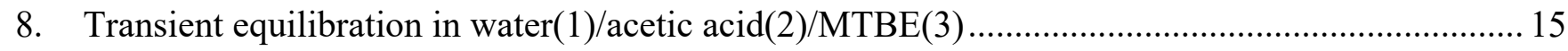

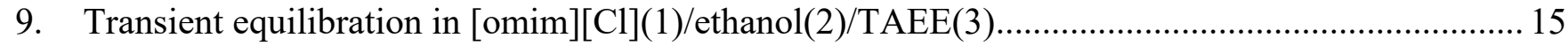

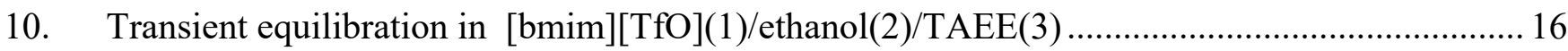

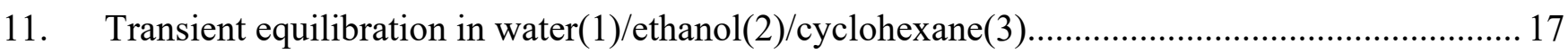

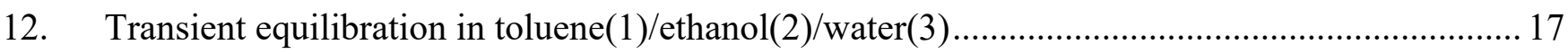

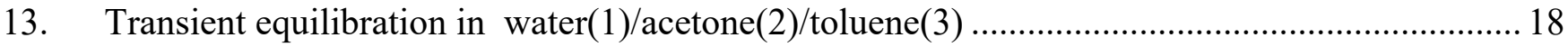

14. Springer experiments for boundary crossing in homogeneous azeotropic distillation................... 18

15. Transient equilibration inside vapor bubble rising through a liquid on a distillation tray .............22

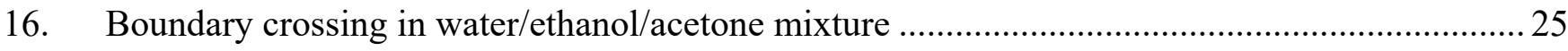

17. NEQ vs EQ models for design of column to separate water/ethanol/acetone mixtures ..................31

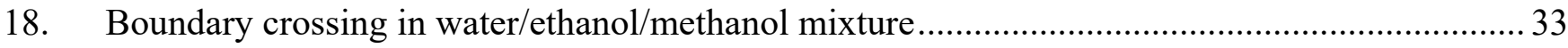

19. Boundary crossing in water/ethanol/methylacetate mixture ........................................................ 38

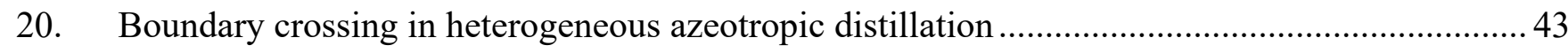

21. Separating azeotropic alcohol/water mixture by diffusional distillation....................................... 44

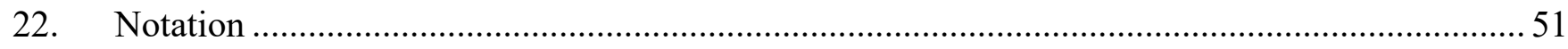

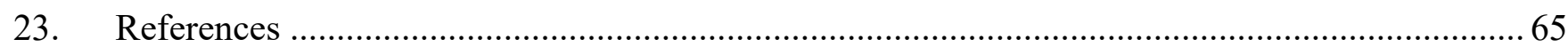

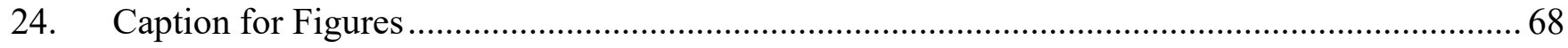




\section{Preamble}

This Supporting Information (SI) accompanying the manuscript Highlighting Diffusional Coupling Effects in Ternary Liquid Extraction and Comparisons with Distillation provides background information on liquid extraction and distillation processes, additional modelling details, input data on diffusivities, tabulated data on the NRTL and UNIQUAC parameters required for the determination of phase equilibrium thermodynamics, along with detailed simulation results (in graphical form) for all systems investigated.

All calculations of Geddes diffusion trajectories, mass transfer coefficients, and efficiencies that are reported in this article involve explicit analytic formulae that were implemented in MathCad $15 .{ }^{1}$ The calculation details are provided herein, in the event the interested reader would like to reproduce our calculations. For ease of reading, this SI is written with some overlap of material with the main manuscript.

\section{Transient equilibration in heptane(1)/toluene(2)/sulpholane(3) mixtures}

Figure 1 is a schematic showing a variety of applications of liquid extraction processes in petroleum refining. Most of these applications involve the separation of aromatics from hydrocarbon mixtures, for example for production of food grade hexane, ${ }^{2}$ improving the smoke point of kerosene, and flow properties of lube oils. There are also other applications in the petrochemical industries. ${ }^{3}$

The contactors used in industry are either agitated, or un-agitated columns; see Figure 2.

Figure 3 is a schematic of single-stage contacting in sieve-tray column. This principle also applies to other stage-wise operations. On a given stage, the continuous liquid phase can be considered to be wellmixed; this is a reasonably good approximation.

For a ternary mixture, the diffusion in either continuous or dispersed phase is described by the generalized Fick's law

$$
(J)=-c_{t}[D] \frac{d(x)}{d z}
$$


in which the two-dimensional matrix of Fick diffusivities $[D]$ is a product of two matrices

$[D]=[\Lambda][\Gamma]$

The $2 \times 2$ matrix of thermodynamic factors $[\Gamma]$

$\frac{x_{i}}{R T} \frac{d \mu_{i}}{d z}=\sum_{j=1}^{2} \Gamma_{i j} \frac{d x_{j}}{d z} ; \quad \Gamma_{i j}=\delta_{i j}+x_{i} \frac{\partial \ln \gamma_{i}}{\partial x_{j}} ; \quad i, j=1,2$

can be calculated from UNIQUAC or NRTL models describing phase equilibrium thermodynamics. ${ }^{4,5}$

The matrix $[\Lambda]$ to be expressed explicitly in terms of the M-S diffusivities of the constituent binary pairs in the ternary mixture:

$\left[\begin{array}{ll}\Lambda_{11} & \Lambda_{12} \\ \Lambda_{21} & \Lambda_{22}\end{array}\right]=\frac{\left[\begin{array}{cc}\bigoplus_{13}\left(x_{1} \bigoplus_{23}+\left(1-x_{1}\right) \bigoplus_{12}\right) & x_{1} \bigoplus_{23}\left(\bigoplus_{13}-\bigoplus_{12}\right) \\ x_{2} \bigoplus_{13}\left(\bigoplus_{23}-\bigoplus_{12}\right) & \bigoplus_{23}\left(x_{2} \bigoplus_{13}+\left(1-x_{2}\right) \bigoplus_{12}\right)\end{array}\right]}{x_{1} \bigoplus_{23}+x_{2} \bigoplus_{13}+x_{3} \bigoplus_{12}}$

For partially miscible ternary mixtures, the coupling effects in the Fick matrix are primarily due to the thermodynamic factors; this has been demonstrated in earlier work. ${ }^{6}$ For all the simulations presented in this article, we use the following, simplified expression for the calculation of the Fick matrix

$[D]=|\Lambda|^{1 / 2}[\Gamma]$

with the scalar diffusivity $|\Lambda|^{1 / 2}$ calculated from

$|\Lambda|^{1 / 2}=\sqrt{\frac{\bigoplus_{12} \bigoplus_{13} \bigoplus_{23}}{x_{1} \bigoplus_{23}+x_{2} \bigoplus_{13}+x_{3} \bigoplus_{12}}}$

Let us consider the dispersion to consist of uniform and rigid droplets of diameter, $d_{d r o p}$. The transient equilibration process within a rigid spherical droplet is described by Geddes model that was originally developed for describing binary diffusion inside vapor bubbles on distillation trays. ${ }^{7}$ For ternary mixtures, the Geddes model can be written in two-dimensional matrix differential equation ${ }^{4}$ 


$$
\left(x-x_{e q}\right)=[Q]\left(x_{0}-x_{e q}\right) ; \quad[Q] \equiv \frac{6}{\pi^{2}} \sum_{m=1}^{\infty} \frac{1}{m^{2}} \exp \left[-m^{2} \pi^{2} \frac{4[D] t}{d_{d r o p}^{2}}\right]
$$

The Sylvester theorem, detailed in Appendix A of Taylor and Krishna, ${ }^{4}$ is required for explicit calculation of the composition trajectories described by Equation (5). For extraction equipment such as a sieve tray or rotating disc contactors, the effective contact time of the dispersed phase droplets with the surrounding continuous phase is $t=h_{f} / V_{\text {drop }}$, where $h_{\mathrm{f}}$ is the liquid/liquid dispersion height, and $V_{\text {drop }}$ is the droplet rise velocity. ${ }^{8}$

The fractional approaches to equilibrium for contact time $t$, also termed as the Murphree efficiencies, ${ }^{3,}$ 9,10 are calculated from

$$
\begin{aligned}
& E_{1}=\frac{x_{10}-x_{1}}{x_{10}-x_{1 e q}}=1-Q_{11}-Q_{12} \frac{\Delta x_{2}}{\Delta x_{1}} ; \\
& E_{2}=\frac{x_{20}-x_{2}}{x_{20}-x_{2 e q}}=1-Q_{22}-Q_{21} \frac{\Delta x_{1}}{\Delta x_{2}} ; \\
& E_{3}=\frac{x_{30}-x_{3}}{x_{30}-x_{3 e q}}=\frac{\frac{\Delta x_{1}}{\Delta x_{2}} E_{1}+E_{2}}{\frac{\Delta x_{1}}{\Delta x_{2}}+1}
\end{aligned}
$$

Let us define a two-dimensional matrix of mass transfer coefficients, $[k]$, defined by

$$
(J)=c_{t}[k]\left(x-x_{e q}\right)
$$

The matrix of Sherwood numbers $[S h]$

$$
\left[S h_{t}\right]=[k] d_{\text {drop }}[D]^{-1}
$$

can be calculated as a function of the contact time using the following expression ${ }^{4}$

$$
\left[S h_{t}\right]=\frac{2}{3} \pi^{2}\left[\sum_{m=1}^{\infty} \exp \left[-m^{2} \pi^{2} \frac{4[D] t}{d_{\text {drop }}^{2}}\right]\right]\left[\frac{6}{\pi^{2}} \sum_{m=1}^{\infty} \frac{1}{m^{2}} \exp \left[-m^{2} \pi^{2} \frac{4[D] t}{d_{\text {drop }}^{2}}\right]\right]^{-1}
$$

In the limit $t \rightarrow \infty$, an asymptotic value is reached 


$$
\left[S h_{t}\right]=\frac{2 \pi^{2}}{3}=6.58
$$

It must be emphasized that Equation (9), is the value of the matrix at any time $t$, and not the timeaveraged value. The time-averaged Sherwood number, $[S h]$, for the interval $0-t$, is given by the expression

$$
[S h]=-\frac{2}{3} \ln [[Q]][D]^{-1} \frac{d_{d r o p}^{2}}{4 t}
$$

In the limit $t \rightarrow \infty$, an asymptotic value is also reached for the time-averaged Sherwood number

$$
[S h]=\frac{2 \pi^{2}}{3}=6.58
$$

The corresponding time-averaged matrix of mass transfer coefficients in the dispersed phase is

$$
\left[k_{d}\right]=\frac{[S h][D]}{d_{\text {drop }}}=-\frac{2}{3} \ln [[Q]][D]^{-1} \frac{d_{\text {drop }}^{2}}{4 t} \frac{[D]}{d_{\text {drop }}}=-\ln [[Q]] \frac{d_{\text {drop }}}{6 t}
$$

In this asymptotic limit $t \rightarrow \infty$, the coupling effects of $[k]$ are directly proportional to those of the Fick matrix $[D]$.

Let us denote the interfacial area per unit volume of drop as

$$
a=\frac{6}{d_{d r o p}}
$$

With this definition, the time-averaged matrix of mass transfer coefficients is

$$
\left[k_{d}\right]=-\ln [[Q]] \frac{1}{a t}
$$

In chemical engineering practice, it is conventional to define the number of transfer units for the dispersed phase as

$$
\left[N T U_{d}\right]=\left[k_{d}\right] a t=-\ln [[Q]]
$$


Therefore an alternative expression for the fractional departure from equilibrium is

$$
[Q]=\exp \left[-N T U_{d}\right]=\exp \left[-\left[k_{d}\right] a t\right]
$$

Let us examine the diffusion equilibration trajectories for heptane(1)/toluene(2)/sulpholane(3) mixtures at $348.2 \mathrm{~K}$. Please note that in the SI we use the English spelling of sulpholane. The US spelling is sulfolane, as used in the main manuscript. In particular, we examine the trajectory followed as droplets containing pure sulpholane (composition: $x_{10}=0.0, x_{20}=0.0$, and $x_{30}=1.0$ ) equilibrates to the equilibrium composition E, at the tie-line indicated in Figure 4: $x_{1, \mathrm{eq}}=0.062684992, x_{2, \mathrm{eq}}=$ 0.406432625 , and $x_{3, \mathrm{eq}}=0.53088$.

The first task is to estimate the M-S diffusivities if the three binary pairs $\bigoplus_{12}, \bigoplus_{13}$, and $\bigoplus_{23}$ using the following interpolation formulation ${ }^{11}$

$\bigoplus_{i j}=\left(\bigoplus_{i j}^{x_{i} \rightarrow 1}\right)^{x_{i}}\left(\bigoplus_{i j}^{x_{j} \rightarrow 1}\right)^{x_{j}}\left(\bigoplus_{i j}^{x_{k} \rightarrow 1}\right)^{x_{k}}$

The six infinite dilution values of the pair diffusivities $\bigoplus_{i j}^{x_{j} \rightarrow 1}$ are estimated the Wilke-Chang correlation. ${ }^{12}$ For estimation of $\bigoplus_{i j}^{x_{k} \rightarrow 1}$, the $i-j$ pair diffusivity when both $i$ and $j$ are present in infinitely dilute concentrations, the following formula has been suggested ${ }^{11}$

$$
\bigoplus_{i j}^{x_{k} \rightarrow 1}=\left(\bigoplus_{i k}^{x_{k} \rightarrow 1}\right)^{x_{i} /\left(x_{i}+x_{j}\right)}\left(\bigoplus_{j k}^{x_{k} \rightarrow 1}\right)^{x_{j} /\left(x_{i}+x_{j}\right)}
$$

At the arithmetic average composition between $\mathrm{S}$ and $\mathrm{E}$, we calculate the following values of the M-S diffusivities of the binary pairs

$$
\bigoplus_{12}=0.94 ; \quad \bigoplus_{13}=0.82 ; \quad \bigoplus_{23}=0.96 \times 10^{-9} \mathrm{~m}^{2} \mathrm{~s}^{-1}
$$

The value of the scalar diffusivity is

$$
|\Lambda|^{1 / 2}=\sqrt{\frac{\bigoplus_{12} \bigoplus_{13} \bigoplus_{23}}{x_{1} \bigoplus_{23}+x_{2} \bigoplus_{13}+x_{3} \bigoplus_{12}}}=0.9 \times 10^{-9} \mathrm{~m}^{2} \mathrm{~s}^{-1}
$$

The matrix of thermodynamic factors is determined from the NRTL parameters provided in Table 1.

$$
[\Gamma]=\left[\begin{array}{cc}
0.71695 & -0.16465 \\
-0.85701 & 0.81773
\end{array}\right]
$$


The Fick matrix is calculated as

$$
[D]=|\Lambda|^{1 / 2}[\Gamma]=\left[\begin{array}{cc}
0.643 & -0.148 \\
-0.769 & 0.733
\end{array}\right] \times 10^{-9}
$$

We can also determine a "magnitude" of the Fick diffusivity for use in the calculation of the Fourier number

$$
|D|^{1 / 2}=0.6 \times 10^{-9}
$$

Let us assume that the droplet of $1 \mathrm{~mm}$ diameter rises at a velocity of $20 \mathrm{~mm} \mathrm{~s}^{-1}$ on a sieve tray with a dispersion height of $400 \mathrm{~mm}$. The contact time, $t=20 \mathrm{~s}$. For this set of input values, we calculate the following set of parameters:

Interfacial area per unit volume of droplet

$$
a=\frac{6}{d_{d r o p}}=6 \times 10^{3}
$$

The matrix describing the departure from equilibrium is

$$
[Q]=\left[\begin{array}{cc}
0.4133 & 0.0527 \\
0.2743 & 0.38105
\end{array}\right]
$$

The matrix for the number of transfer units for the dispersed phase

$$
\left[N T U_{d}\right]=\left[\begin{array}{cc}
0.93041 & -0.13707 \\
-0.713443 & 1.01431
\end{array}\right]
$$

The time-average matrix of mass transfer coefficients is

$$
\left[k_{d}\right]=\left[\begin{array}{cc}
7.75341 & -1.1422 \\
-5.94533 & 8.45255
\end{array}\right] \times 10^{-6}
$$

The component Murphree efficiencies are

$$
\begin{aligned}
& E_{1}=\frac{x_{10}-x_{1}}{x_{10}-x_{1 e q}}=1-Q_{11}-Q_{12} \frac{\Delta x_{2}}{\Delta x_{1}}=0.245 \\
& E_{2}=\frac{x_{20}-x_{2}}{x_{20}-x_{2 e q}}=1-Q_{22}-Q_{21} \frac{\Delta x_{1}}{\Delta x_{2}}=0.577 \\
& E_{3}=\frac{x_{30}-x_{3}}{x_{30}-x_{3 e q}}=\frac{\frac{\Delta x_{1}}{\Delta x_{2}} E_{1}+E_{2}}{\frac{\Delta x_{1}}{\Delta x_{2}}+1}=0.532
\end{aligned}
$$


The toluene/heptane ratio in dispersed phase leaving the stage at contact time of $20 \mathrm{~s}$ can be calculated from

$$
\left(x-x_{e q}\right)=[Q]\left(x_{0}-x_{e q}\right) ; \quad[Q]=\exp \left[-\left[K_{o d}\right] a t\right]
$$

The value is calculated as $\frac{0.23437}{0.01536}=15.26$.

The Kronig-Brink ${ }^{13}$ model that takes account of circulation within the droplet, leads to the following expression for the departure from equilibrium

$$
\left(x-x_{e q}\right)=[Q]\left(x_{0}-x_{e q}\right) ; \quad[Q] \equiv \frac{3}{8} \sum_{m=1}^{\infty} A_{m}^{2} \exp \left[-16 \lambda_{m} \frac{4[D] t}{d_{d r o p}^{2}}\right]
$$

The values of $A_{m}$ and $\lambda_{m}$ are tabulated by Sideman and Shabtai; ${ }^{14}$ see Table 2. Equation (20) predicts a more rapid equilibration than the rigid sphere model (5). In composition space, the diffusion equilibration trajectory calculated using equation (20) follows a curvilinear path that is practically indistinguishable from that predicted by the Geddes model for equilibration.

We now examine the influence of the resistance to interphase mass transfer offered by the continuous phase, i.e. the raffinate. Figure 27 presents a schematic of mass transfer resistances in liquid-liquid extraction.

All the calculations of the parameters for the raffinate phase are an average between the compositions HT $\left(x_{10}=0.25, x_{20}=0.75\right.$, and $\left.x_{30}=0.0\right)$ and the final equilibrium compositions of $\mathrm{R}\left(x_{1, \mathrm{eq}}=\right.$ $0.258578176, x_{2, \mathrm{eq}}=0.621464559$, and $\left.x_{3, \mathrm{eq}}=0.11996\right)$, as indicated in Figure 4.

At the arithmetic average composition between $\mathrm{S}$ and $\mathrm{E}$, we calculate the M-S diffusivities of the binary pairs

$$
\bigoplus_{12}=2.8 ; \quad \bigoplus_{13}=2.55 ; \quad \bigoplus_{23}=2.95 \times 10^{-9} \mathrm{~m}^{2} \mathrm{~s}^{-1}
$$

The scalar diffusivity value is

$$
|\Lambda|^{1 / 2}=\sqrt{\frac{\bigoplus_{12} \bigoplus_{13} \bigoplus_{23}}{x_{1} \bigoplus_{23}+x_{2} \bigoplus_{13}+x_{3} \bigoplus_{12}}}=2.58 \times 10^{-9} \mathrm{~m}^{2} \mathrm{~s}^{-1}
$$

The matrix of thermodynamic factors is calculated from the NRTL parameters 
$[\Gamma]=\left[\begin{array}{cc}0.46322 & -0.646785 \\ -0.017851 & 1.243143\end{array}\right]$

The Fick matrix for the continuous (raffinate) phase is

$[D]=|\Lambda|^{1 / 2}[\Gamma]=\left[\begin{array}{cc}1.19536 & -1.669058 \\ -0.04607 & 3.20799\end{array}\right] \times 10^{-9}$

The magnitude of the Fick matrix is

$|D|^{1 / 2}=1.94 \times 10^{-9}$

It is noticeable that all diffusivity values for the raffinate phase are higher than the corresponding ones for the extract phase; the primary reason is the lower concentration of sulpholane in the raffinate phase.

The mass transfer coefficient external to the droplet can be estimated from the surface renewal theory $^{15,16}$

$\left[k_{c}\right]=2 \sqrt{\frac{\left[D_{c}\right]}{\pi t_{\text {renewal }}}}$

The Fick matrix $\left[D_{c}\right]$ is evaluated using $\left[D_{c}\right]=\left|\Lambda_{c}\right|^{1 / 2}\left[\Gamma_{c}\right]$ at the average between the compositions of HT and R. The characteristic time for surface renewal can be estimated is $t_{\text {renewal }}=d_{d r o p} / V_{\text {drop }}=0.05 \mathrm{~s}$; this value is significantly lower than the time the droplet takes to rise through the dispersion, $20 \mathrm{~s}$.

For a surface renewal time of $0.05 \mathrm{~s}$, the mass transfer in the continuous phase can be estimated from the surface renewal theory

$$
\left[k_{c}\right]=2 \sqrt{\frac{[D]}{\pi t_{\text {renewal }}}}=\left[\begin{array}{cc}
173.79033 & -92.55819 \\
-2.55464 & 285.40194
\end{array}\right] \times 10^{-6}
$$

The overall mass transfer coefficient can be calculated

$$
\left[K_{O d}\right]^{-1}=\left[k_{d}\right]^{-1}+\frac{c_{t L, d}}{c_{t L, c}}\left[\begin{array}{cc}
\frac{x_{1 d, e q}}{x_{1 c, e q}} & 0 \\
0 & \frac{x_{2 d, e q}}{x_{2 c, e q}}
\end{array}\right]\left[k_{c}\right]^{-1}
$$


Inserting the values of the partial mass transfer coefficients, we have for the overall mass transfer coefficients

$$
\begin{aligned}
& {\left[K_{\text {Od }}\right]^{-1}=} {\left[\begin{array}{cc}
7.75341 & -1.1422 \\
-5.945333 & 8.45255
\end{array}\right]^{-1} \times 10^{6}+} \\
& \frac{10132}{8981}\left[\begin{array}{cc}
\frac{0.062684992}{0.258578176} & 0 \\
0 & \frac{0.406432625}{0.621464559}
\end{array}\right]\left[\begin{array}{rr}
173.79033 & -92.55819 \\
-2.55464 & 285.40194
\end{array}\right]^{-1} \times 10^{6} \\
& {\left[K_{\text {Od }}\right]=\left[\begin{array}{cc}
7.66559 & -1.13673 \\
-5.76671 & 8.28557
\end{array}\right] \times 10^{-6} }
\end{aligned}
$$

The matrix for the overall number of transfer units

$$
\left[N T U O_{d}\right]=\left[\begin{array}{cc}
0.91987 & -0.13641 \\
-0.69201 & 0.99427
\end{array}\right]
$$

The Murphree component efficiencies are

$$
\begin{aligned}
& E_{1}=\frac{x_{10}-x_{1}}{x_{10}-x_{1 e q}}=1-Q_{11}-Q_{12} \frac{\Delta x_{2}}{\Delta x_{1}}=0.23785 \\
& E_{2}=\frac{x_{20}-x_{2}}{x_{20}-x_{2 e q}}=1-Q_{22}-Q_{21} \frac{\Delta x_{1}}{\Delta x_{2}}=0.57032 \\
& E_{3}=\frac{x_{30}-x_{3}}{x_{30}-x_{3 e q}}=\frac{\frac{\Delta x_{1}}{\Delta x_{2}} E_{1}+E_{2}}{\frac{\Delta x_{1}}{\Delta x_{2}}+1}=0.52589
\end{aligned}
$$

The toluene/heptane ratio in dispersed phase leaving the stage at contact of $0.05 \mathrm{~s}$ can be calculated from

$$
\left(x-x_{e q}\right)=[Q]\left(x_{0}-x_{e q}\right), \quad[Q]=\exp \left[-\left[K_{o d}\right] a t\right]
$$

The value is calculated as $\frac{0.2318}{0.01491}=15.54$. The toluene/heptane ratio is very slightly higher than the corresponding value obtained by ignoring the continuous phase mass transfer resistances. 


\section{Transient equilibration in NMP(1)/propylbenzene(2)/tetradecane(3)}

The solvent NMP (=N-methyl-2-pyrrolidone) is dispersed as droplets in the hydrocarbon phase. Figure 6 shows the equilibration trajectory. The trajectory calculations are obtained from the following input data (see also legend to Figure 6).

$$
\begin{aligned}
& |\Lambda|^{1 / 2}=1 \times 10^{-9} \mathrm{~m}^{2} \mathrm{~s}^{-1} \\
& {[\Gamma]=\left[\begin{array}{cc}
0.05136 & -0.91061 \\
0.45016 & 1.68594
\end{array}\right]} \\
& {[D]=|\Lambda|^{1 / 2}[\Gamma]=\left[\begin{array}{cc}
0.05136 & -0.91061 \\
0.45016 & 1.68594
\end{array}\right] \times 10^{-9}} \\
& |D|^{1 / 2}=7 \times 10^{-10}
\end{aligned}
$$

\section{Transient equilibration in glycerol(1)/acetone(2)/water(3)}

The experimental data on transient equilibration of glycerol-rich and acetone-rich phases of the glycerol/acetone/water mixture were measured in a stirred Lewis cell by Krishna et al. ${ }^{17}$ see Figure 7. In this case, the interface between the two liquid phases is flat; the appropriate expression for the departure from equilibrium in either the glycerol-rich or the acetone-rich phase is ${ }^{17}$

$$
\left(x-x_{e q}\right)=[Q]\left(x_{0}-x_{e q}\right), \quad[Q] \equiv \exp [-\beta[D] t]
$$

where $\beta$ is the Lewis cell constant. The value of the constant used in our calculations is $\beta=10^{7}$. The precise choice of the value of this constant has no influence on the trajectories in composition space.

The start and end compositions in the glycerol-rich and acetone-rich phase are those in the experiments of Krishna et al..; ${ }^{17}$ see legend to Figure 7. The two trajectories are calculated using $[D]=|\Lambda|^{1 / 2}[\Gamma]$ with $|\Lambda|^{1 / 2}=\left(D_{1, \text { self }}\right)^{x_{1}}\left(D_{2, \text { self }}\right)^{x_{2}}\left(D_{3, \text { self }}\right)^{x_{3}}$, taking $D_{1, \text { self }}=0.01, D_{2, \text { self }}=3.2, D_{3, \text { self }}=0.5$ with units $10^{-9} \mathrm{~m}^{2} \mathrm{~s}^{-1}$; this diffusivity information has been derived from our earlier work. ${ }^{6}$

For the glycerol-rich phase the following input data are calculated:

$$
|\Lambda|^{1 / 2}=3.75 \times 10^{-11} \mathrm{~m}^{2} \mathrm{~s}^{-1}
$$


$[\Gamma]=\left[\begin{array}{ll}2.151617 & 1.171495 \\ 0.019193 & 0.687462\end{array}\right]$

$[D]=|\Lambda|^{1 / 2}[\Gamma]=\left[\begin{array}{cc}8.069819 & 4.39379 \\ 0.071986 & 2.578383\end{array}\right] \times 10^{-11}$

$|D|^{1 / 2}=4.53 \times 10^{-11}$

For the acetone-rich phase the following input data are calculated:

$$
\begin{aligned}
& |\Lambda|^{1 / 2}=2.23 \times 10^{-9} \mathrm{~m}^{2} \mathrm{~s}^{-1} \\
& {[\Gamma]=\left[\begin{array}{cc}
1.064438 & 0.177947 \\
0.9564073 & 0.766246
\end{array}\right]} \\
& {[D]=|\Lambda|^{1 / 2}[\Gamma]=\left[\begin{array}{cc}
2.296962 & 0.383994 \\
2.06384 & 1.653491
\end{array}\right] \times 10^{-9}} \\
& |D|^{1 / 2}=1.73 \times 10^{-9}
\end{aligned}
$$

\section{Transient equilibration in water(1)/acetone(1)/ethyl-acetate(3)}

Figure 8 and Figure 9 show the transient equilibration trajectories for the system water(1)/acetone(2)/ethylacetate(3) at $293 \mathrm{~K}$.

The trajectory calculations in Figure 8 are obtained from the following input data (see also legend to Figure 8).

$$
\begin{aligned}
& |\Lambda|^{1 / 2}=2.14 \times 10^{-9} \mathrm{~m}^{2} \mathrm{~s}^{-1} \\
& {[\Gamma]=\left[\begin{array}{cc}
0.335504 & -0.287411 \\
-5.6 \times 10^{-3} & 1.233074
\end{array}\right]} \\
& {[D]=|\Lambda|^{1 / 2}[\Gamma]=\left[\begin{array}{cc}
0.717637 & -0.614767 \\
-0.012094 & 2.637524
\end{array}\right] \times 10^{-9}} \\
& |D|^{1 / 2}=1.37 \times 10^{-9}
\end{aligned}
$$




\section{Transient equilibration in water(1)/caprolactam(2)/toluene(3)}

Figure 10 and Figure 11 show the transient equilibration trajectories for the system water(1)/caprolactam(2)/toluene(3) at $298 \mathrm{~K}$.

The trajectory calculations in Figure 10 are obtained from the following input data (see also legend to Figure 10).

$$
\begin{aligned}
& |\Lambda|^{1 / 2}=1 \times 10^{-9} \mathrm{~m}^{2} \mathrm{~s}^{-1} \\
& {[\Gamma]=\left[\begin{array}{cc}
0.9312464 & -0.1564682 \\
-0.9630304 & 0.6333638
\end{array}\right]} \\
& {[D]=|\Lambda|^{1 / 2}[\Gamma]=\left[\begin{array}{cc}
0.9312464 & -0.1564682 \\
-0.9630304 & 0.6333638
\end{array}\right] \times 10^{-9}} \\
& |D|^{1 / 2}=6.63 \times 10^{-10}
\end{aligned}
$$

\section{Transient equilibration in water(1)/acetic acid(2)/isophorone(3)}

Figure 12 and Figure 13 show the transient equilibration trajectories for the system water(1)/acetic $\operatorname{acid}(2) /$ isophorone(3) at $298 \mathrm{~K}$.

The trajectory calculations in Figure 12 are obtained from the following input data (see also legend to Figure 12).

$$
\begin{aligned}
& |\Lambda|^{1 / 2}=1 \times 10^{-9} \mathrm{~m}^{2} \mathrm{~s}^{-1} \\
& {[\Gamma]=\left[\begin{array}{cc}
0.7352077 & -0.47604686 \\
-0.13916565 & 1.38146886
\end{array}\right]} \\
& {[D]=|\Lambda|^{1 / 2}[\Gamma]=\left[\begin{array}{cc}
0.7352077 & -0.47604686 \\
-0.13916565 & 1.38146886
\end{array}\right] \times 10^{-9}} \\
& |D|^{1 / 2}=9.74 \times 10^{-10}
\end{aligned}
$$




\section{Transient equilibration in water(1)/acetic acid(2)/MTBE(3)}

Figure 14 and Figure 15 show the transient equilibration trajectories for the system water(1)/acetic $\operatorname{acid}(2) / \operatorname{MTBE}(3)$ at $298.15 \mathrm{~K}$.

The trajectory calculations in Figure 14 are obtained from the following input data (see also legend to Figure 14).

$$
\begin{aligned}
& |\Lambda|^{1 / 2}=1 \times 10^{-10} \mathrm{~m}^{2} \mathrm{~s}^{-1} \\
& {[\Gamma]=\left[\begin{array}{cc}
0.33765906 & -0.75846144 \\
-0.26661839 & 1.75009021
\end{array}\right]} \\
& {[D]=|\Lambda|^{1 / 2}[\Gamma]=\left[\begin{array}{cc}
0.33765906 & -0.75846144 \\
-0.26661839 & 1.75009021
\end{array}\right] \times 10^{-9}} \\
& |D|^{1 / 2}=6.2 \times 10^{-10}
\end{aligned}
$$

\section{Transient equilibration in [omim][CI](1)/ethanol(2)/TAEE(3)}

There is increasing amount of fundamental and technological interest in the use of ionic liquids in separations in liquid-liquid extraction. We shall examine the diffusion trajectories in ternary mixtures of $[\mathrm{omim}][\mathrm{Cl}](1) /$ ethanol(2)/TAEE(3). Here we denote the ionic liquid 1-octyl-3-methylimidazolium chloride in the abbreviated form [omim][Cl]. TAEE is the abbreviated name for tert-amyl ethyl ether.

Figure 16, Figure 17, and Figure 18 show the transient equilibration trajectory for the system [omim][Cl](1)/ethanol(2)/TAEE(3) at 298.15 K.

The trajectory calculations in Figure 16 are obtained from the following input data (see also legend to Figure 16). The final equilibrium composition is $x_{1, \mathrm{eq}}=0.150072736, x_{2, \mathrm{eq}}=0.47026855$, and $x_{3, \mathrm{eq}}=$ 0.379658714 .

$$
\begin{aligned}
& |\Lambda|^{1 / 2}=1 \times 10^{-10} \mathrm{~m}^{2} \mathrm{~s}^{-1} \\
& {[\Gamma]=\left[\begin{array}{ll}
1.155617836 & -2.5088582 \\
-0.71005985 & 3.20938546
\end{array}\right]}
\end{aligned}
$$




$$
\begin{aligned}
& {[D]=|\Lambda|^{1 / 2}[\Gamma]=\left[\begin{array}{ll}
1.155617836 & -2.5088582 \\
-0.71005985 & 3.20938546
\end{array}\right] \times 10^{-10}} \\
& |D|^{1 / 2}=1.4 \times 10^{-10}
\end{aligned}
$$

The trajectory calculations in Figure 17 are obtained from the following input data (see also legend to Figure 17). The final equilibrium composition is $x_{1, \mathrm{eq}}=0.397919131, x_{2, \mathrm{eq}}=0.381690314$, and $x_{3, \mathrm{eq}}=$ 0.22039056 .

$$
\begin{aligned}
& |\Lambda|^{1 / 2}=1 \times 10^{-10} \mathrm{~m}^{2} \mathrm{~s}^{-1} \\
& {[\Gamma]=\left[\begin{array}{cc}
1.04814698 & -2.42905971 \\
-0.40060012 & 3.22825358
\end{array}\right]} \\
& {[D]=|\Lambda|^{1 / 2}[\Gamma]=\left[\begin{array}{cc}
1.04814698 & -2.42905971 \\
-0.40060012 & 3.22825358
\end{array}\right] \times 10^{-10}} \\
& |D|^{1 / 2}=1.55 \times 10^{-10}
\end{aligned}
$$

\section{Transient equilibration in [bmim][TfO](1)/ethanol(2)/TAEE(3)}

We also investigation the diffusion trajectories using a different ionic liquid [bmim $][\mathrm{TfO}]=1$-butyl3-methylimidazolium trifluoromethanesulfonate.

Figure 19 and Figure 20 the transient equilibration trajectories for the system [bmim][TfO](1)/ethanol(2)/TAEE(3) at $298.15 \mathrm{~K}$.

The trajectory calculations in Figure 19 are obtained from the following input data (see also legend to Figure 19).

$$
\begin{aligned}
& |\Lambda|^{1 / 2}=1 \times 10^{-10} \mathrm{~m}^{2} \mathrm{~s}^{-1} \\
& {[\Gamma]=\left[\begin{array}{cc}
0.6048285 & -1.8612996 \\
0.0978455 & 3.1563742
\end{array}\right]} \\
& {[D]=|\Lambda|^{1 / 2}[\Gamma]=\left[\begin{array}{cc}
0.6048285 & -1.8612996 \\
0.0978455 & 3.1563742
\end{array}\right] \times 10^{-10}} \\
& |D|^{1 / 2}=1.44 \times 10^{-10}
\end{aligned}
$$




\section{Transient equilibration in water(1)/ethanol(2)/cyclohexane(3)}

Figure 21 and Figure 22 show the transient equilibration trajectory for the system water(1)/ethanol(2)/cyclohexane(3) at $298 \mathrm{~K}$.

The trajectory calculations in Figure 21 are obtained from the following input data (see also legend to Figure 21).

$$
\begin{aligned}
& |\Lambda|^{1 / 2}=3.13 \times 10^{-9} \mathrm{~m}^{2} \mathrm{~s}^{-1} \\
& {[\Gamma]=\left[\begin{array}{cc}
0.08686 & -0.49724 \\
0.33949 & 1.04153
\end{array}\right]} \\
& {[D]=|\Lambda|^{1 / 2}[\Gamma]=\left[\begin{array}{ll}
0.27222 & -1.5584 \\
1.06401 & 3.42367
\end{array}\right] \times 10^{-9}} \\
& |D|^{1 / 2}=1.6 \times 10^{-9}
\end{aligned}
$$

\section{Transient equilibration in toluene(1)/ethanol(2)/water(3)}

Figure 23 and Figure 24 show the transient equilibration trajectory for the system toluene(1)/ethanol(2)/water(3) at $298 \mathrm{~K}$.

The trajectory calculations in Figure 23 are obtained from the following input data (see also legend to Figure 23).

$$
\begin{aligned}
& |\Lambda|^{1 / 2}=1 \times 10^{-9} \mathrm{~m}^{2} \mathrm{~s}^{-1} \\
& {[\Gamma]=\left[\begin{array}{cc}
0.17105226 & -0.32119791 \\
0.41999696 & 1.14665716
\end{array}\right]} \\
& {[D]=|\Lambda|^{1 / 2}[\Gamma]=\left[\begin{array}{cc}
0.17105226 & -0.32119791 \\
0.41999696 & 1.14665716
\end{array}\right] \times 10^{-9}} \\
& |D|^{1 / 2}=5.75 \times 10^{-10}
\end{aligned}
$$




\section{Transient equilibration in water(1)/acetone(2)/toluene(3)}

Figure 25 and Figure 26 show the transient equilibration trajectory for the system water(1)/acetone(2)/toluene(3) at $298 \mathrm{~K}$.

The trajectory calculations in Figure 25 are obtained from the following input data (see also legend to Figure 25).

$$
\begin{aligned}
& |\Lambda|^{1 / 2}=1 \times 10^{-9} \mathrm{~m}^{2} \mathrm{~s}^{-1} \\
& {[\Gamma]=\left[\begin{array}{cc}
-0.303576 & -0.636406 \\
1.12334 & 1.593812
\end{array}\right]} \\
& {[D]=|\Lambda|^{1 / 2}[\Gamma]=\left[\begin{array}{cc}
-0.303576 & -0.636406 \\
1.12334 & 1.593812
\end{array}\right] \times 10^{-9}} \\
& |D|^{1 / 2}=4.8 \times 10^{-10}
\end{aligned}
$$

\section{Springer experiments for boundary crossing in homogeneous azeotropic distillation}

Design and simulation procedures for distillation are commonly based on the equilibrium stage model, developed by Sorel more than a hundred years ago. ${ }^{18}$ Departures from thermodynamic equilibrium between the vapor and liquid phases on a distillation tray are commonly accounted for by introducing the component Murphree point efficiencies

$$
E_{i}=\frac{y_{i E}-y_{i L}}{y_{i E}-y_{i, e q}}=1-\frac{y_{i L}-y_{i, e q}}{y_{i E}-y_{i, e q}}=1-\frac{\Delta y_{i L}}{\Delta y_{i E}} ; \quad i=1,2, \ldots n
$$

where $y_{i E}$, and $y_{i L}$ are, respectively, the vapor phase mole fractions, entering and leaving a tray, and $y_{i, e q}$ is the vapor composition in thermodynamic equilibrium with the liquid leaving the tray. See schematic in Figure 27. For a tray in thermodynamic equilibrium, the component efficiencies are $100 \%$ for each component. Mass transfer resistances on either side of the vapor/liquid interface reduce the component efficiencies to values below $100 \%$. For binary distillation, the Murphree component efficiencies are bounded, i.e. $0 \leq E_{1,}=E_{2} \leq 1$. For multicomponent distillation, with the number of 
species $n \geq 3$, coupled diffusion effects in either vapor or liquid phases cause the component efficiencies to be distinctly different from one another, $E_{1} \neq E_{2} \neq E_{3}$. Phenomena such as osmotic diffusion, diffusion barrier, and uphill diffusion lead to component efficiencies that are unbounded $\left(E_{i} \rightarrow \pm \infty\right)$, zero $\left(E_{i}=0\right)$, or negative $\left(E_{i}<0\right)$; this has been demonstrated in several experimental studies. $^{19-27}$

The values of the component Murphree efficiencies influence the composition profiles along the height of distillation columns.

Levy et al. ${ }^{28}$ have put forward the following two "rules" regarding that are applicable to continuous azeotropic distillation columns operating with each stage in thermodynamic equilibrium:

- If the simple distillation boundary is perfectly linear, then the steady-state composition profile in a continuous distillation column cannot cross the boundary from either side.

- If the simple distillation boundary is curved, then the steady-state composition profile in a continuous distillation column cannot cross the boundary from the concave side but may cross the boundary from the convex side when moving from the product compositions inward.

Consider, for example, the system methanol - isopropanol - water; the residue curve maps for this system are shown in Figure 28a. A straight-line distillation boundary connects the binary isopropanolwater azeotrope with pure methanol and divides the composition space into two regions. According to Rule 1, the column composition trajectories cannot cross this straight line distillation boundary, whichever side the feed is located. For either of the two feed locations, F1 and F2 in Figure 28a boundary crossing is forbidden.

For the system acetone - chloroform - methanol we have three binary and one ternary azeotrope dividing the composition space into four regions by means of four distillation boundaries, that are all curved; see the residue curve map shown in Figure 28b. According to Rule 2, the column trajectory obtained for operation with the feed located on the concave side of a boundary, with say composition indicated by F1 is able to cross that boundary. This has been demonstrated experimentally by Li et al. ${ }^{29}$ 
Conversely, if the feed is located on the convex side, with say composition indicated by F2 the boundary cannot be crossed. ${ }^{28}$

In a series of papers, Springer et al. ${ }^{21,23,25,26}$ have reported a set of experiments in a bubble-cap tray column operating at total reflux for homogeneous azeotropic distillation using mixtures: water/ethanol/methanol, water/ethanol/acetone, water/ethanol/methylacetate, water/ethanol/methanol/acetone to demonstrate that the Levy rules are violated. The experimental set-up used by Springer can be viewed at: http://krishna.amsterchem.com/distillation/. A schematic of the experimental set-up is shown in Figure 29. The set-up consists of a 12-stage distillation column wherein all the experiments were conducted under total-reflux conditions at $101.3 \mathrm{kPa}$. The condenser is a total condenser, and is considered to be stage 1 . The numbering of the stages is downwards, and the Stage 12 is the partial reboiler. Stages 2, to 11 are bubble-cap trays.

As an example of boundary crossing in homogeneous azeotropic distillation, we present the experimental results for Run T2-26 for water(1)/ethanol(2)/acetone(3) mixture in Figure 30, In Run T226 , the condenser composition is left of the distillation boundary. Therefore, the residue curves dictate that the reboiler composition should be in the top left corner, rich in ethanol. The measured compositions along the column operating at total reflux shows that the reboiler composition is towards the right of the distillation boundary, and is rich in water. Boundary crossing occurs at stage 2 , just below the total condenser.

For Run T2-26, the values of $\Delta y_{2 E}=\left(y_{2 E}-y_{2, e q}\right)$ are plotted in Figure 31a. We note that the ethanol driving force $\Delta y_{2 E}=\left(y_{2 E}-y_{2, e q}\right)>0$ for Stages $2-9, \Delta y_{2 E}=\left(y_{2 E}-y_{2, e q}\right) \approx 0$ for Stage 10 , and $\Delta y_{2 E}=\left(y_{2 E}-y_{2, e q}\right)<0$ for Stage 11. The values of the $E_{2}$ for ethanol is negative on Stage 10; on Stage 11, $E_{i}>1$; see Figure $31 \mathrm{~b}$. This implies reverse or uphill diffusion on stages 10 and 11; the transfer of ethanol is dictated by the driving forces of the other two components $\Delta y_{1 E}=\left(y_{1 E}-y_{1, e q}\right)$, and $\Delta y_{3 E}=\left(y_{3 E}-y_{3, e q}\right)$, that are both finite..$^{21}$ 
We shall demonstrate later that the boundary crossing is primarily due to the factor that the Murphree efficiency of water is higher than that of ethanol, i.e. $E_{1}>E_{2}$.

The experimental data for Run T4-13 with water(1)/ethanol(2)/methanol(3) mixture are shown in Figure 32, In Run T4-13, the condenser composition is left of the distillation boundary. Therefore, the residue curves dictate that the reboiler composition should be in the top left corner, rich in ethanol. The measured compositions along the column operating at total reflux shows that the reboiler composition is towards the right of the distillation boundary, and is rich in water. Also shown in Figure 32 are the Murphree component efficiencies along the column for Run T4-13. We shall demonstrate later that the boundary crossing is primarily due to the factor that the Murphree efficiency of water is higher than that of ethanol, i.e. $E_{1}>E_{2}$.

The experimental data for Run T3-23 with water(1)/ethanol(2)/methylacetate(3) mixture are shown in Figure 33. In Run T3-23, the condenser composition is left of the distillation boundary. Therefore, the residue curves dictate that the reboiler composition should be in the top left corner, rich in ethanol. The measured compositions along the column operating at total reflux shows that the reboiler composition is towards the right of the distillation boundary, and is rich in water. Also shown in Figure 33 are the Murphree component efficiencies along the column for Run T4-13. We shall demonstrate later that the boundary crossing is primarily due to the factor that the Murphree efficiency of water is higher than that of ethanol, i.e. $E_{1}>E_{2}$.

Experimental data (blue circles) of Springer et al. ${ }^{25}$ for Run Q6 with quaternary water(1)/ethanol(2)/methanol(3)/acetone(4) mixtures are indicated by the blue circles in Figure 34; the data are plotted in ternary composition space by combining the mole fractions of methanol and acetone in the left bottom vertex. Two distillation boundaries are shown: the "acetone" boundary is the same as for the water/ethanol/acetone mixture; the "methanol" boundary is the same as for the water/ethanol/methanol mixture. The experimental data shows that both the "acetone" and "methanol" boundaries are crossed in Run Q6. Also shown as insets are the Murphree component efficiencies and component driving forces. The component Murphree efficiency of methanol is negative on stage 3, and 
slightly exceeds unity on stage 4. This implies that uphill diffusion of methanol manifests on stages 3

and 4. The reason is to found in the fact that the driving force of methanol is practically zero on these two stages; the direction of transport of methanol is dictated by the transfer of the three partner species in the mixture: water, ethanol, and acetone. The boundary crossing is primarily due to the factor that the Murphree efficiency of water is higher than that of ethanol, i.e. $E_{1}>E_{2}$.

For rationalization and quantitative description of the observed experimental boundary crossing phenomena, Springer et al. Springer et al. ${ }^{21,23,25,26}$ used rigorous non-equilibrium (NEQ) stage-wise contacting model, as implemented in ChemSep. ${ }^{18,} 30$ The NEQ model uses the Maxwell-Stefan formulation for diffusion in the vapor and the two liquid phases. The important conclusion reached in their work is that boundary crossing effects are primarily attributable to diffusional coupling effects, that cause the component Murphree efficiencies to be unequal to one another. Unequal component efficiencies cause column composition trajectories to deviate from those of the residue curve maps. Put another way, the NEQ model does not follow the tramline guides of the RCM.

Our aim below is to show that the boundary crossing phenomena can be explained on the basis of the Geddes model for transient equilibration of vapor bubbles rising through the liquid on a tray.

\section{Transient equilibration inside vapor bubble rising through a liquid on a distillation tray}

For a ternary mixture, the diffusion, in either the dispersed vapor bubbles or in the continuous liquid phase surrounding the bubbles, is described by the generalized Fick's law

$$
(J)=-c_{t}[D] \frac{d(x)}{d z}
$$

in which the two-dimensional matrix of Fick diffusivities $[D]$ is a product of two matrices

$[D]=[\Lambda][\Gamma]$

The vapor phase can often be considered to thermodynamically ideal; in this event, the matrix of thermodynamic factor degenerates to the identity matrix 
$\Gamma_{i j}=\delta_{i j} ; \quad i, j=1,2$

The matrix $[\Lambda]$ to be expressed explicitly in terms of the M-S diffusivities of the constituent binary pairs in the ternary mixture:

$\left[\begin{array}{ll}\Lambda_{11} & \Lambda_{12} \\ \Lambda_{21} & \Lambda_{22}\end{array}\right]=\frac{\left[\begin{array}{cc}\bigoplus_{13}\left(x_{1} \bigoplus_{23}+\left(1-x_{1}\right) \bigoplus_{12}\right) & x_{1} \bigoplus_{23}\left(\bigoplus_{13}-\bigoplus_{12}\right) \\ x_{2} \bigoplus_{13}\left(\bigoplus_{23}-\bigoplus_{12}\right) & \bigoplus_{23}\left(x_{2} \bigoplus_{13}+\left(1-x_{2}\right) \bigoplus_{12}\right)\end{array}\right]}{x_{1} \bigoplus_{23}+x_{2} \bigoplus_{13}+x_{3} \bigoplus_{12}}$

Let us consider the dispersion to consist of uniform and rigid vapor bubbles of diameter, $d_{b u b b l e}$. The transient equilibration process within a rigid spherical bubble is described by Geddes model that was originally developed for describing binary diffusion inside vapor bubbles on distillation trays. ${ }^{7}$ For ternary mixtures, the Geddes model can be written in two-dimensional matrix differential equation ${ }^{4,31}$

$$
\left(y-x_{e q}\right)=[Q]\left(y_{0}-x_{e q}\right), \quad[Q] \equiv \frac{6}{\pi^{2}} \sum_{m=1}^{\infty} \frac{1}{m^{2}} \exp \left[-m^{2} \pi^{2} \frac{4[D] t}{d_{b u b b l e}^{2}}\right]
$$

In the above equation, $\left(y_{0}\right)$ denotes the vapor composition entering the tray. The Sylvester theorem, detailed in Appendix A of Taylor and Krishna, ${ }^{4}$ is required for explicit calculation of the composition trajectories described by the Geddes model. For vapor bubbles rising on a sieve or bubble-cap tray, the effective contact time of the dispersed phase bubbles with the surrounding continuous phase is $t=h_{f} / V_{\text {bubble }}$, where $h_{\mathrm{f}}$ is the froth dispersion height, and $V_{\text {bubble }}$ is the bubble rise velocity.

The fractional approaches to equilibrium for contact time $t$, also termed as the Murphree efficiencies, ${ }^{3,}$ 9,10 are calculated from

$$
\begin{aligned}
& E_{1}=\frac{y_{10}-y_{1}}{y_{10}-y_{1 e q}}=1-Q_{11}-Q_{12} \frac{\Delta y_{2}}{\Delta y_{1}} ; \\
& E_{2}=\frac{y_{20}-y_{2}}{y_{20}-y_{2 e q}}=1-Q_{22}-Q_{21} \frac{\Delta y_{1}}{\Delta y_{2}} ; \\
& E_{3}=\frac{y_{30}-y_{3}}{y_{30}-y_{3 e q}}=\frac{\frac{\Delta y_{1}}{\Delta y_{2}} E_{1}+E_{2}}{\frac{\Delta y_{1}}{\Delta y_{2}}+1}
\end{aligned}
$$


Let us define a two-dimensional matrix of mass transfer coefficients, $[k]$, defined by

$(J)=c_{t}[k]\left(y-y_{e q}\right)$

The matrix of Sherwood numbers $[S h]$

$\left[S h_{t}\right]=[k] d_{\text {bubble }}[D]^{-1}$

can be calculated as a function of the contact time using the following expression ${ }^{4}$

$\left[S h_{t}\right]=\frac{2}{3} \pi^{2}\left[\sum_{m=1}^{\infty} \exp \left[-m^{2} \pi^{2} \frac{4[D] t}{d_{\text {bubble }}^{2}}\right]\right]\left[\frac{6}{\pi^{2}} \sum_{m=1}^{\infty} \frac{1}{m^{2}} \exp \left[-m^{2} \pi^{2} \frac{4[D] t}{d_{\text {bubble }}^{2}}\right]\right]^{-1}$

In the limit $t \rightarrow \infty$, an asymptotic value is reached

$\left[S h_{t}\right]=\frac{2 \pi^{2}}{3}=6.58$

It must be emphasized that $\left[S h_{t}\right]$ is the value of the matrix at any time $t$, and not the time-averaged value. The time-averaged Sherwood number, $[S h]$, for the interval $0-t$, is given by the expression

$[S h]=-\frac{2}{3} \ln [[Q]][D]^{-1} \frac{d_{\text {bubble }}^{2}}{4 t}$

In the limit $t \rightarrow \infty$, an asymptotic value is also reached for the time-averaged Sherwood number

$[S h]=\frac{2 \pi^{2}}{3}=6.58$

The corresponding time-averaged matrix of mass transfer coefficients in the dispersed vapor phase is

$\left[k_{d}\right]=\frac{[S h][D]}{d_{\text {bubble }}}=-\frac{2}{3} \ln [[Q]][D]^{-1} \frac{d_{\text {bubble }}^{2}}{4 t} \frac{[D]}{d_{b}}=-\ln [[Q]] \frac{d_{\text {bubble }}}{6 t}$

In this asymptotic limit $t \rightarrow \infty$, the coupling effects of $[k]$ are directly proportional to those of the Fick matrix $[D]$.

Let us denote the interfacial area per unit volume of bubble as 
$a=\frac{6}{d_{\text {bubble }}}$

With this definition, the time-averaged matrix of mass transfer coefficients is

$\left[k_{d}\right]=-\ln [[Q]] \frac{1}{a t}$

In chemical engineering practice, it is conventional to define the number of transfer units for the dispersed phase as

$\left[N T U_{d}\right]=\left[k_{d}\right] a t=-\ln [[Q]]$

Therefore an alternative expression for the fractional departure from equilibrium is

$[Q]=\exp \left[-N T U_{d}\right]=\exp \left[-\left[k_{d}\right] a t\right]$

We now apply the Geddes model to rationalize the boundary effects for for

water(1)/ethanol(2)/acetone(3), $\quad$ water(1)/ethanol(2)/methanol(3), and water(1)/ethanol(2)/methylacetate(3) mixtures.

\section{Boundary crossing in water/ethanol/acetone mixture}

Consider distillation of water(1)/ethanol(2)/acetone(3) mixture in a tray column operating at total reflux at a total pressure of $101.3 \mathrm{kPa}$. For a specified tray, the composition of the vapor entering the tray is $y_{10}=0.067, y_{20}=0.44$, and $y_{30}=0.493$. This composition is right of the distillation boundary. For total reflux operations, the compositions of the liquid leaving that stage will be equal to that of the vapor entering the stage, i.e. $x_{1}=0.067, x_{2}=0.44$, and $x_{3}=0.493$. The composition of vapor in equilibrium with the liquid leaving the tray can be determined using the NRTL parameters provided in Table 15. The bubble point temperature is $335.5 \mathrm{~K}$ and the equilibrium composition is $y_{1, \mathrm{eq}}=0.04335, y_{2, \mathrm{eq}}=$ 0.25907 , and $y_{3, \mathrm{eq}}=0.69758$. The equilibrium composition is also right of the distillation boundary, as is to be expected. 
The driving forces are $\Delta y_{1}=y_{10}-y_{1, e q}=0.02365$, and $\Delta y_{2}=y_{20}-y_{2, e q}=0.18093$. Both driving forces are positive, i.e. directed from vapor to the liquid phase.

The values of the vapor phase M-S diffusivities of the binary pairs, calculated using the FullerSchettler-Giddings $(\mathrm{FSG})^{32}$ method, are $\bigoplus_{12}=1.97 ; \bigoplus_{13}=1.71 ; \bigoplus_{23}=0.851 \times 10^{-5} \mathrm{~m}^{2} \mathrm{~s}^{-1}$. These diffusivities are independent of composition. The differences in the binary pair diffusivities cannot be ignored, as we demonstrate below. At the average composition between the entering compositions and the equilibrated compositions, use of

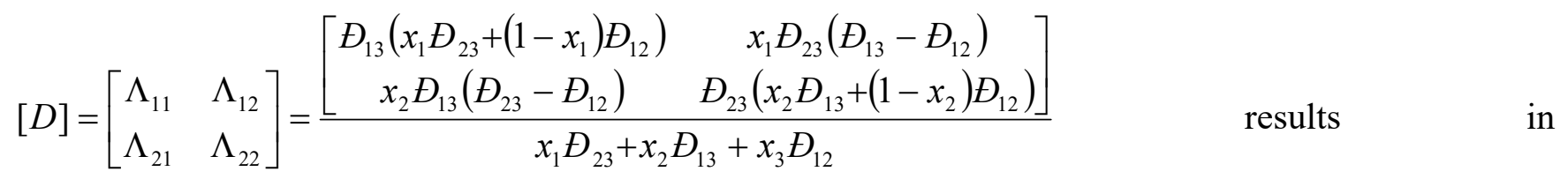
$[D]=\left[\begin{array}{cc}1.79675 & -0.0067 \\ -0.36845 & 0.87989\end{array}\right] \times 10^{-5} \mathrm{~m}^{2} \mathrm{~s}^{-1}$ in which the $D_{21}$ is seen to non-negligible in comparison with $D_{22}$

We can also determine a "magnitude" of the Fick diffusivity for use in the calculation of the Fourier number: $|D|^{1 / 2}=1.26 \times 10^{-5}$ in order to plot the results in terms of dimensionless times.

The diffusion equilibration trajectory, calculated using the Geddes model is shown in Figure 35a. The curvilinear equilibration trajectory crosses the distillation boundary during a portion of this traject. Figure $35 \mathrm{~b}$ presents a plot of the component Murphree efficiencies, $E_{\mathrm{i}}$, as function of the Fourier number. The Murphree point efficiency of ethanol, $E_{2}$, is the lowest; this is because of the negative contribution of $D_{12} \Delta y_{2}$; the Murphree point efficiency of water, $E_{1}$, is higher than that of ethanol: $E_{1}>$ $E_{2}$. Due to $E_{1}>E_{2}$, a higher proportion of water is transferred to the liquid phase as compared to ethanol; this implies that the vapor phase is poorer in water than predicted by calculations based on equal component efficiencies. The hierarchy of point efficiencies $E_{1}>E_{2} \approx E_{3}$ is in agreement with the experimentally determined values for Stage 2; see Figure 31 b. 
The contact time of the bubble with the liquid phase is finite. For a $4.5 \mathrm{~mm}$ bubble, with a rise velocity of $0.2 \mathrm{~m} \mathrm{~s}^{-1}$ in a dispersion of height $9.2 \mathrm{~mm}$, the contact time $t=0.046 \mathrm{~s}$; these are the input parameters used by Springer et al. in the NEQ model implementation. ${ }^{23}$

For this contact time, the composition of the vapor bubble leaving the tray is $y_{1}=0.0465, y_{2}=0.3118$, and $y_{3}=0.64175$.

This vapor composition is on the other side of the distillation boundary. Such boundary crossing is observed in Run T2-26 of the experiments of Springer et al. ${ }^{21,23,25,26}$ (cf. Figure 30).

The time-averaged mass transfer coefficient for the dispersed bubbles, for contact time $t=0.046 \mathrm{~s}$ is $\left[k_{d}\right]=-\ln [Q] \frac{d_{\text {bubble }}}{6 t}$

For $t=0.046 \mathrm{~s}$, we have the matrix describing the departure from equilibrium is

$$
[Q]=\left[\begin{array}{cc}
0.12178 & 1.17915 \times 10^{-3} \\
0.06477 & 0.38105
\end{array}\right]
$$

and

$$
\left[k_{d}\right]=\left[\begin{array}{cc}
343.52901 & -1.00639 \\
-55.27758 & 205.97396
\end{array}\right] \times 10^{-4}
$$

If the diffusion coupling effects are completely ignored, and the simplest Fickian relation

$$
J_{i}=-c_{t} D \frac{d x_{i}}{d z} ; \quad i=1,2,3
$$

is employed, the equilibration trajectory will be linear, running parallel to the residue curves (pink lines) in Figure 35a.

Let us examine what influence inclusion of liquid phase mass transfer resistance has on the composition trajectories.

The first task is to estimate the M-S diffusivities if the three binary pairs $\bigoplus_{12}, \bigoplus_{13}$, and $\bigoplus_{23}$ in the liquid phase using the interpolation formula

$$
\bigoplus_{i j}=\left(\bigoplus_{i j}^{x_{i} \rightarrow 1}\right)^{x_{i}}\left(\bigoplus_{i j}^{x_{j} \rightarrow 1}\right)^{x_{j}}\left(\bigoplus_{i j}^{x_{k} \rightarrow 1}\right)^{x_{k}}
$$


The six infinite dilution values of the pair diffusivities $\bigoplus_{i j}^{x_{j} \rightarrow 1}$ are estimated the Wilke-Chang correlation. ${ }^{12}$ For estimation of $\bigoplus_{i j}^{x_{k} \rightarrow 1}$, the $i-j$ pair diffusivity when both $i$ and $j$ are present in infinitely dilute concentrations, the following formula has been suggested ${ }^{11}$ $\bigoplus_{i j}^{x_{k} \rightarrow 1}=\left(\bigoplus_{i k}^{x_{k} \rightarrow 1}\right)^{x_{i} /\left(x_{i}+x_{j}\right)}\left(\bigoplus_{j k}^{x_{k} \rightarrow 1}\right)^{x_{j} /\left(x_{i}+x_{j}\right)}$

At the liquid composition on the tray: $x_{1}=0.067, x_{2}=0.44$, and $x_{3}=0.493$, we calculate the following values of the M-S diffusivities of the binary pairs

$$
\bigoplus_{12}=6.4 ; \quad \bigoplus_{13}=5.6 ; \quad \bigoplus_{23}=3.8 \times 10^{-9} \mathrm{~m}^{2} \mathrm{~s}^{-1}
$$

For estimation of the Fick diffusivity, we use the approximation ${ }^{6}$

$[D]=|\Lambda|^{1 / 2}[\Gamma]$

The value of the scalar diffusivity is

$$
|\Lambda|^{1 / 2}=\sqrt{\frac{\bigoplus_{12} \bigoplus_{13} \Theta_{23}}{x_{1} \bigoplus_{23}+x_{2} \Theta_{13}+x_{3} \Theta_{12}}}=4.8 \times 10^{-9} \mathrm{~m}^{2} \mathrm{~s}^{-1}
$$

The matrix of thermodynamic factors is

$$
[\Gamma]=\left[\begin{array}{cc}
0.86952 & -0.03937 \\
-0.20988 & 0.75351
\end{array}\right]
$$

The Fick diffusivity matrix in the liquid phase is calculated as

$$
[D]=|\Lambda|^{1 / 2}[\Gamma]=\left[\begin{array}{cc}
4.16901 & -0.18874 \\
-1.00627 & 3.61281
\end{array}\right] \times 10^{-9}
$$

The mass transfer coefficient external to the bubble can be estimated from the surface renewal theory ${ }^{15,16}$

$$
\left[k_{c}\right]=2 \sqrt{\frac{[D]}{\pi t_{\text {renewal }}}}
$$


The characteristic time for surface renewal can be estimated is $t_{\text {renewal }}=d_{\text {bubble }} / V_{\text {bubble }}=0.0225 \mathrm{~s}$; this value is lower than the time the bubble takes to rise through the dispersion, $0.046 \mathrm{~s}$.

For a surface renewal time of $0.0225 \mathrm{~s}$, the mass transfer in the continuous liquid phase can be estimated from the surface renewal theory (cf. schematic in Figure 27)

$$
\left[k_{c}\right]=2 \sqrt{\frac{[D]}{\pi t_{\text {renewal }}}}=\left[\begin{array}{cc}
4.84999 & -0.11406 \\
-0.60812 & 4.51386
\end{array}\right] \times 10^{-4}
$$

The overall mass transfer coefficient can be calculated from the addition of resistances formula

$$
\left[K_{O d}\right]^{-1}=\left[k_{d}\right]^{-1}+\frac{c_{t V, d}}{c_{t L, c}}\left[\begin{array}{cc}
\frac{y_{1, e q}}{x_{1}} & 0 \\
0 & \frac{y_{2, e q}}{x_{2}}
\end{array}\right]\left[k_{c}\right]^{-1}
$$

Inserting the values of the partial mass transfer coefficients, we have for the overall mass transfer coefficients

$$
\begin{aligned}
& {\left[K_{\text {Od }}\right]^{-1}=} {\left[\begin{array}{cc}
343.52901 & -1.00639 \\
-55.27758 & 205.97396
\end{array}\right]^{-1} \times 10^{4}+} \\
& \frac{36.32105}{1.48899 \times 10^{4}}\left[\begin{array}{cc}
\frac{0.04335}{0.067} & 0 \\
0 & \frac{0.25907}{0.44}
\end{array}\right]\left[\begin{array}{cc}
4.84999 & -0.11406 \\
-0.60812 & 4.51386
\end{array}\right]^{-1} \times 10^{4}
\end{aligned}
$$

The matrix of overall mass transfer coefficients is

$$
\left[K_{\text {Od }}\right]=\left[\begin{array}{cc}
309.00758 & -1.34179 \\
-49.04598 & 193.33745
\end{array}\right] \times 10^{-4}
$$

We note that $\left[K_{O d}\right] \approx\left[k_{d}\right]$, indicating that the mass transfer is controlled by diffusion within the vapor bubbles; the liquid phase mass transfer resistance is of negligible importance.

The matrix for the overall number of transfer units can be calculated from

$$
\left[N T U_{O d}\right]=\left[K_{O d}\right] a t
$$

The interfacial area per unit volume of bubble is

$$
a=\frac{6}{d_{\text {bubble }}}=1.333 \times 10^{3}
$$


Therefore, for contact time $t=0.046 \mathrm{~s}$ is

$$
\left[N T U_{O \mathrm{~d}}\right]=\left[\begin{array}{cc}
1.89525 & -0.00823 \\
-0.30082 & 0.99427
\end{array}\right]
$$

The matrix describing departure from equilibrium can be determined from

$$
\begin{aligned}
& {[Q]=\exp \left[-\left[N T U_{O d}\right]\right]} \\
& {[Q]=\left[\begin{array}{cc}
0.15052 & 1.8013 \times 10^{-3} \\
0.06584 & 0.3058
\end{array}\right]}
\end{aligned}
$$

The composition of the vapor bubble exiting the tray at $t=0.046 \mathrm{~s}$ is determined from

$$
\left(y-x_{e q}\right)=[Q]\left(y_{0}-x_{e q}\right)
$$

The calculated value is

$$
y_{1}=0.04724, y_{2}=0.31596 \text {, and } y_{3}=0.6368 \text {. }
$$

This vapor composition is very close to the value calculated above by ignoring the liquid phase resistance. Boundary crossing persists even if we ignore the liquid phase mass transfer resistance. The Geddes provides an accurate prediction of boundary crossing of water(1)/ethanol(2)/acetone(3) mixture.

For various vapor compositions entering any given stage, we have plotted in Figure 35c the actual composition vector $\left(y_{i, L}-y_{i, E}\right)$, calculated from the NEQ model (taking bubble diameter of $4.5 \mathrm{~mm}$ ) along with the equilibrium vector $\left(y_{i}^{*}-y_{i, E}\right)$. The angle between the NEQ trajectory (red line) and the EQ trajectory (blue line) increases when the differences in the component efficiencies increase. If all the component efficiencies were equal to one another, the NEQ and EQ trajectories would coincide. We see from Figure 35c that the NEQ trajectory has a tendency to cut across to the right of the EQ trajectory, precisely as has been observed in Run T2-26; cf. Figure 30. It is this tendency to cut towards the right of the composition space that causes boundary crossing. By performing several NEQ simulations with various starting compositions of the vapor entering the condenser we can determine the region within which the column trajectories will cross the distillation boundary and end up with reboiler compositions in the right region. This boundary crossing region is shown as the orange shaded area in Figure 35d. 


\section{NEQ vs EQ models for design of column to separate water/ethanol/acetone mixtures}

The design of a distillation column to meet the required specification with regard to product purity is distinctively different to that of simulation as discussed above. In this section, our objective is to demonstrate the inclusion of diffusional coupling effects will lead to different requirements on the number of trays, in order to achieve a desired level of purity, when compared to a conventional approach of using equal component efficiencies. The material presented in this section are taken from an unpublished manuscript (P.A.M. Springer, R. Baur, R. Krishna, Influence of Diffusional Coupling on Distillation Column Design, 2004), that is based on the contents of Chapter 9 of the Ph.D. Dissertation of Springer. ${ }^{33}$ The chosen mixture for investigation is water(1)/ethanol(2)/acetone(3) that has been investigated by Springer in the context of boundary crossing. The column hardware details are tabulated in Table 16. The distillation column was maintained at a total pressure of $101.3 \mathrm{kPa}$ and the ideal gas law was applied. The vapor pressures were calculated using the Antoine equation. The reflux ratio = 3; this is an optimum value reached after an optimization study. The bottoms product flow rate was specified as $1.75 \mathrm{~mol} / \mathrm{s}$. The chosen parameters are summarized in Table 17.

Two different feed compositions are chosen, either side of the distillation boundary.

For feed on the left side of the distillation boundary, the feed was introduced in such a way that $20 \%$ of all column-stages were located above the feed stage. In this case, a purity level of $96 \%$ ethanol in the bottom product is specified.

For feed on the right side of the distillation boundary, the feed tray was located exactly halfway through the distillation column. In this case, a purity level of $100 \%$ water in the bottom product is specified.

The simulations were carried out using ChemSep developed by Kooijman and Taylor. ${ }^{30}$ The ChemSep manual, that can be downloaded online, contains details of all thermodynamics, hydrodynamics and mass transfer models for tray columns that have been implemented into the software. The mass transfer coefficients for the NEQ stage model were estimated using the AIChE 1958 
correlation for sieve tray columns with inclusion of the liquid phase mass transfer resistance. Both the vapor and liquid were assumed to be well-mixed. No pressure drop or entrainment was considered. The equilibrium data (NRTL parameters) for the water - ethanol - acetone system are specified in Table 18. For comparison purposes, simulations were also carried out with and EQ model assume that all the trays have the same efficiency, and that all component efficiencies are identical. The choice of the efficiency value is chosen as the average of that calculated by the NEQ model, averaged over all the trays.

Let us first consider the results for feed on the distillation region on the left side of the distillation boundary for the system water (1) - ethanol (2) - acetone (3); see Figure 36. For the purity requirement of $96 \%$ ethanol in the reboiler, the NEQ model requires 39 stages; see Figure 36b. The EQ model (with equal component efficiencies) requires only 25 stages to attain this product purity. The reason for the extra stages required by the NEQ is evident on examination of the column composition trajectory in Figure 36a. The NEQ trajectory appears to veer towards the water vertex because of the higher component efficiency of water (Figure 36c). Water is the least volatile of the three components, and its transfer is directed from vapor to the liquid phase; a higher efficiency of water ensures that the liquid phase is richer in water than anticipated on the basis of equal component efficiencies.

For the feed mixture right of the distillation boundary, the results are shown in Figure 37 . In this case, the NEQ model requires 30 stages to reach $100 \%$ water purity in the bottoms product; the EQ model (with equal component efficiencies) requires 38 trays to reach the same purity target; see Figure 37b. The corresponding composition trajectories, plotted in Figure 37a, the higher number of stages required by the EQ model is because it takes the "scenic" route in proceeding to the bottom of the column. Due to the higher component efficiency of water (see Figure 37c), the composition trajectory followed by the NEQ model veers towards the water vertex and consequently demands fewer number of stages. In other words, the NEQ model takes a shorter route, and the EQ model follows the scenic route in the column. 
The overall conclusion to be drawn from our design study is that use of the conventional EQ model may be either overly optimistic or pessimistic, depending on the which side of the distillation column the operation is taking place.

\section{Boundary crossing in water/ethanol/methanol mixture}

Consider distillation of water(1)/ethanol(2)/methanol(3) mixture in a tray column operating at total reflux at a total pressure of $101.3 \mathrm{kPa}$. For a specified tray, the composition of the vapor entering the tray is $y_{10}=0.082, y_{20}=0.68$, and $y_{30}=0.238$. This composition is right of the distillation boundary. For total reflux operations, the compositions of the liquid leaving that stage will be equal to that of the vapor entering the stage, i.e. $x_{1}=0.082, x_{2}=0.68$, and $x_{3}=0.238$. The composition of vapor in equilibrium with the liquid leaving the tray can be determined using the NRTL parameters provided in Table 15. The bubble point temperature is $348 \mathrm{~K}$ and the equilibrium composition is $y_{1, \mathrm{eq}}=0.06767$, $y_{2, \mathrm{eq}}=0.59691$, and $y_{3, \mathrm{eq}}=0.33542$. The final equilibrated composition is also right of the distillation boundary, as is to be expected.

The driving forces are $\Delta y_{1}=y_{10}-y_{1, e q}=0.01433$, and $\Delta y_{2}=y_{20}-y_{2, e q}=0.08309$. Both driving forces are positive, i.e. directed from vapor to the liquid phase.

The values of the vapor phase M-S diffusivities of the binary pairs, calculated using the FullerSchettler-Giddings (FSG) $)^{32}$ method, are $\bigoplus_{12}=2.1 ; \bigoplus_{13}=2.72 ; \quad \bigoplus_{23}=1.36 \times 10^{-5} \mathrm{~m}^{2} \mathrm{~s}^{-1}$. These diffusivities are independent of composition. The differences in the binary pair diffusivities cannot be ignored, as we demonstrate below. At the average composition between the entering compositions and the equilibrated compositions, use of

$[D]=\left[\begin{array}{cc}\Lambda_{11} & \Lambda_{12} \\ \Lambda_{21} & \Lambda_{22}\end{array}\right]=\frac{\left[\begin{array}{cc}\bigoplus_{13}\left(x_{1} \bigoplus_{23}+\left(1-x_{1}\right) \bigoplus_{12}\right) & x_{1} \bigoplus_{23}\left(\bigoplus_{13}-\bigoplus_{12}\right) \\ x_{2} \bigoplus_{13}\left(\bigoplus_{23}-\bigoplus_{12}\right) & \bigoplus_{23}\left(x_{2} \bigoplus_{13}+\left(1-x_{2}\right) \bigoplus_{12}\right)\end{array}\right]}{x_{1} \bigoplus_{23}+x_{2} \bigoplus_{13}+x_{3} \bigoplus_{12}} \quad$ results
$[D]=\left[\begin{array}{cc}2.28276 & 0.02578 \\ -0.52706 & 1.39446\end{array}\right] \times 10^{-5} \mathrm{~m}^{2} \mathrm{~s}^{-1}$ in which the $D_{21}$ is seen to non-negligible in comparison with 
$D_{22}$. We can also determine a "magnitude" of the Fick diffusivity for use in the calculation of the

Fourier number: $|D|^{1 / 2}=1.8 \times 10^{-5}$ in order to plot the results in terms of dimensionless times.

The diffusion equilibration trajectory, calculated using the Geddes model is shown in Figure 38a.

Figure $38 \mathrm{~b}$ presents a plot of the component Murphree efficiencies, $E_{\mathrm{i}}$, as function of the Fourier number. The curvilinear equilibration trajectory crosses the distillation boundary during a portion of this traject. The Murphree point efficiency of ethanol, $E_{2}$, is the lowest; this is because of the negative contribution of $D_{12} \Delta y_{2}$; the Murphree point efficiency of water, $E_{1}$, is higher than that of ethanol: $E_{1}>$ $E_{2}$. Due to $E_{1}>E_{2}$, a higher proportion of water is transferred to the liquid phase as compared to ethanol; this implies that the vapor phase is poorer in water than predicted by calculations based on equal component efficiencies.

The contact time of the bubble with the liquid phase is finite. For a $4.5 \mathrm{~mm}$ bubble, with a rise velocity of $0.2 \mathrm{~m} \mathrm{~s}^{-1}$ in a dispersion of height $9.2 \mathrm{~mm}$, the contact time $t=0.046 \mathrm{~s}$; these are the input parameters used by Springer et al. ${ }^{21,23,25,26}$ in the NEQ model implementation. ${ }^{23}$

For this contact time, the composition of the vapor bubble leaving the tray is $y_{1}=0.06856, y_{2}=0.61221$, and $y_{3}=0.31943$.

This vapor composition is on the other side of the distillation boundary. Such boundary crossing is observed in Run T4-13 of the experiments of Springer et al. ${ }^{23}$ (cf. Figure 32).

The time-averaged mass transfer coefficient for the dispersed bubbles, for contact time $t=0.046 \mathrm{~s}$ is

$$
\left[k_{d}\right]=-\ln [Q] \frac{d_{b u b b l e}}{6 t}
$$

For $t=0.046 \mathrm{~s}$, we have the matrix describing the departure from equilibrium is

$$
[Q]=\left[\begin{array}{cc}
0.07794 & -2.797 \times 10^{-3} \\
0.05719 & 0.17432
\end{array}\right]
$$

and

$$
\left[k_{d}\right]=\left[\begin{array}{cc}
414.846671 & 3.79431 \\
-77.57228 & 284.10861
\end{array}\right] \times 10^{-4}
$$


If the diffusion coupling effects are completely ignored, and the simplest Fickian relation

$J_{i}=-c_{t} D \frac{d x_{i}}{d z} ; \quad i=1,2,3$

is employed, the equilibration trajectory will be linear, running parallel to the residue curves (pink lines) in Figure 38a.

Let us examine what influence inclusion of liquid phase mass transfer resistance has on the composition trajectories.

At the liquid composition on the tray: $x_{1}=0.082, x_{2}=0.68$, and $x_{3}=0.238$, we calculate the following values of the M-S diffusivities of the binary pairs

$$
\bigoplus_{12}=6.2 ; \quad \bigoplus_{13}=5.6 ; \quad \bigoplus_{23}=4.1 \times 10^{-9} \mathrm{~m}^{2} \mathrm{~s}^{-1}
$$

For estimation of the Fick diffusivity, we use the approximation ${ }^{6}$

$[D]=|\Lambda|^{1 / 2}[\Gamma]$

The value of the scalar diffusivity is

$$
|\Lambda|^{1 / 2}=\sqrt{\frac{\bigoplus_{12} \bigoplus_{13} \bigoplus_{23}}{x_{1} \bigoplus_{23}+x_{2} \bigoplus_{13}+x_{3} \bigoplus_{12}}}=5 \times 10^{-9} \mathrm{~m}^{2} \mathrm{~s}^{-1}
$$

The matrix of thermodynamic factors is

$$
[\Gamma]=\left[\begin{array}{ll}
0.95488 & 0.03852 \\
0.13448 & 0.98609
\end{array}\right]
$$

The Fick diffusivity matrix in the liquid phase is calculated as

$$
[D]=|\Lambda|^{1 / 2}[\Gamma]=\left[\begin{array}{cc}
4.79312 & 0.193364 \\
0.67501 & 4.94981
\end{array}\right] \times 10^{-9}
$$

The mass transfer coefficient external to the bubble can be estimated from the surface renewal theory $^{15,16}$

$$
\left[k_{c}\right]=2 \sqrt{\frac{[D]}{\pi t_{\text {renewal }}}}
$$


The characteristic time for surface renewal can be estimated is $t_{\text {renewal }}=d_{\text {bubble }} / V_{\text {bubble }}=0.0225 \mathrm{~s}$; this value is lower than the time the bubble takes to rise through the dispersion, $0.046 \mathrm{~s}$.

For a surface renewal time of $0.0225 \mathrm{~s}$, the mass transfer in the continuous liquid phase can be estimated from the surface renewal theory

$$
\left[k_{c}\right]=2 \sqrt{\frac{[D]}{\pi t_{\text {renewal }}}}=\left[\begin{array}{ll}
5.20438 & 0.10428 \\
0.36402 & 5.28888
\end{array}\right] \times 10^{-4}
$$

The overall mass transfer coefficient can be calculated from the addition of resistances formula

$$
\left[K_{O d}\right]^{-1}=\left[k_{d}\right]^{-1}+\frac{c_{t V, d}}{c_{t L, c}}\left[\begin{array}{cc}
\frac{y_{1, e q}}{x_{1}} & 0 \\
0 & \frac{y_{2, e q}}{x_{2}}
\end{array}\right]\left[k_{c}\right]^{-1}
$$

Inserting the values of the partial mass transfer coefficients, we have for the overall mass transfer coefficients

$$
\begin{aligned}
{\left[K_{\text {Od }}\right]^{-1}=} & {\left[\begin{array}{cc}
414.846671 & 3.79431 \\
-77.57228 & 284.10861
\end{array}\right]^{-1} \times 10^{4}+} \\
& \frac{34.73188}{1.83151 \times 10^{4}}\left[\begin{array}{cc}
\frac{0.06767}{0.082} & 0 \\
0 & \frac{0.59691}{0.68}
\end{array}\right]\left[\begin{array}{cc}
5.20438 & 0.10428 \\
0.36402 & 5.28888
\end{array}\right]^{-1} \times 10^{4}
\end{aligned}
$$

The matrix of overall mass transfer coefficients is

$$
\left[K_{O d}\right]=\left[\begin{array}{cc}
368.73607 & 3.66694 \\
-61.14586 & 260.74857
\end{array}\right] \times 10^{-4}
$$

We note that $\left[K_{O d}\right] \approx\left[k_{d}\right]$, indicating that the mass transfer is controlled by diffusion within the vapor bubbles; the liquid phase mass transfer resistance is of negligible importance.

The matrix for the overall number of transfer units can be calculated from

$$
\left[N T U_{O d}\right]=\left[K_{O d}\right] a t
$$

The interfacial area per unit volume of bubble is

$$
a=\frac{6}{d_{\text {bubble }}}=1.333 \times 10^{3}
$$


Therefore, for contact time $t=0.046 \mathrm{~s}$ is

$$
\left[N T U_{O \mathrm{~d}}\right]=\left[\begin{array}{cc}
2.26158 & 0.02249 \\
-0.37503 & 1.59926
\end{array}\right]
$$

The matrix describing departure from equilibrium can be determined from

$$
\begin{aligned}
& {[Q]=\exp \left[-\left[N T U_{O d}\right]\right]} \\
& {[Q]=\left[\begin{array}{cc}
0.10363 & -3.31843 \times 10^{-3} \\
0.05533 & 0.20136
\end{array}\right]}
\end{aligned}
$$

The composition of the vapor bubble exiting the tray at $t=0.046 \mathrm{~s}$ is determined from

$$
\left(y-x_{e q}\right)=[Q]\left(y_{0}-x_{e q}\right)
$$

The calculated value is

$$
y_{1}=0.06888, y_{2}=0.61443 \text {, and } y_{3}=0.31668 \text {. }
$$

This vapor composition is very close to the value calculated above by ignoring the liquid phase resistance. Boundary crossing persists even if we ignore the liquid phase mass transfer resistance. The Geddes provides an accurate prediction of boundary crossing for water(1)/ethanol(2)/methanol(3) mixture.

For various vapor compositions entering any given stage, we have plotted in Figure 38c the actual composition vector $\left(y_{i, L}-y_{i, E}\right)$, calculated from the NEQ model (taking bubble diameter of $4.5 \mathrm{~mm}$ ) along with the equilibrium vector $\left(y_{i}^{*}-y_{i, E}\right)$. The angle between the NEQ trajectory (red line) and the EQ trajectory (blue line) increases when the differences in the component efficiencies increase. If all the component efficiencies were equal to one another, the NEQ and EQ trajectories would coincide. We see from Figure 38c that the NEQ trajectory has a tendency to cut across to the right of the EQ trajectory, precisely as has been observed in Run T4-13; cf. Figure 32. It is this tendency to cut towards the right of the composition space that causes boundary crossing. By performing several NEQ simulations with various starting compositions of the vapor entering the condenser we can determine the region within which the column trajectories will cross the distillation boundary and end up with reboiler 
compositions in the right region. This boundary crossing region is shown as the orange shaded area in Figure 38d.

\section{Boundary crossing in water/ethanol/methylacetate mixture}

Consider distillation of water(1)/ethanol(2)/methylacetatel(3) mixture in a tray column operating at total reflux at a total pressure of $101.3 \mathrm{kPa}$. For a specified tray, the composition of the vapor entering the tray is $y_{10}=0.095, y_{20}=0.6345$, and $y_{30}=0.2705$. The chosen vapor composition is right of the distillation boundary. For total reflux operations, the compositions of the liquid leaving that stage will be equal to that of the vapor entering the stage, i.e. $x_{1}=0.095, x_{2}=0.6345$, and $x_{3}=0.2705$. The composition of vapor in equilibrium with the liquid leaving the tray can be determined using the NRTL parameters provided in Table 15. The bubble point temperature is $337 \mathrm{~K}$ and the equilibrium composition is $y_{1, \mathrm{eq}}=0.06324, y_{2, \mathrm{eq}}=0.36863$, and $y_{3, \mathrm{eq}}=0.56813$. The final equilibrated composition is also right of the distillation boundary, as is to be expected.

The driving forces are $\Delta y_{1}=y_{10}-y_{1, e q}=0.03176$, and $\Delta y_{2}=y_{20}-y_{2, e q}=0.26587$.

The values of the vapor phase M-S diffusivities of the binary pairs, calculated using the FullerSchettler-Giddings $(\mathrm{FSG})^{32}$ method, are $\bigoplus_{12}=2 ; \bigoplus_{13}=1.62 ; \quad \bigoplus_{23}=7.91 \times 10^{-5} \mathrm{~m}^{2} \mathrm{~s}^{-1}$. These diffusivities are independent of composition. The differences in the binary pair diffusivities cannot be ignored, as we demonstrate below. At the average composition between the entering compositions and

the equilibrated compositions, use of

$[D]=\left[\begin{array}{cc}\Lambda_{11} & \Lambda_{12} \\ \Lambda_{21} & \Lambda_{22}\end{array}\right]=\frac{\left[\begin{array}{cc}\bigoplus_{13}\left(x_{1} \bigoplus_{23}+\left(1-x_{1}\right) \bigoplus_{12}\right) & x_{1} \bigoplus_{23}\left(\bigoplus_{13}-\bigoplus_{12}\right) \\ x_{2} \bigoplus_{13}\left(\bigoplus_{23}-\bigoplus_{12}\right) & \bigoplus_{23}\left(x_{2} \bigoplus_{13}+\left(1-x_{2}\right) \bigoplus_{12}\right)\end{array}\right]}{x_{1} \bigoplus_{23}+x_{2} \bigoplus_{13}+x_{3} \bigoplus_{12}} \quad$ results
$[D]=\left[\begin{array}{cc}1.79479 & -0.01346 \\ -0.56871 & 0.83543\end{array}\right] \times 10^{-5} \mathrm{~m}^{2} \mathrm{~s}^{-1}$ in which the $D_{21}$ is seen to non-negligible in comparison with $D_{22}$. We can also determine a "magnitude" of the Fick diffusivity for use in the calculation of the Fourier number: $|D|^{1 / 2}=1.22 \times 10^{-5}$ in order to plot the results in terms of dimensionless times. 
The diffusion equilibration trajectory, calculated using Geddes model is shown in Figure 39a. Figure $39 \mathrm{~b}$ presents a plot of the component Murphree efficiencies, $E_{\mathrm{i}}$, as function of the Fourier number. The curvilinear equilibration trajectory crosses the distillation boundary during a portion of this traject.

The Murphree point efficiency of ethanol is the lowest; this is because of the negative contribution of the $D_{12} \Delta y_{2}$. A lower amount of ethanol is transferred to the liquid phase than predicted by an uncoupled equation; i.e. the vapor phase is richer in ethanol. The component efficiency of water is higher than that of partner species; see Figure 39b. Water is the least volatile of the three components, and its transfer is directed from vapor to the liquid phase; a higher efficiency of water ensures that the liquid phase is richer in water than anticipated on the basis of equal component efficiencies.

The contact time of the bubble with the liquid phase is finite. For a $4.5 \mathrm{~mm}$ bubble, with a rise velocity of $0.2 \mathrm{~m} \mathrm{~s}^{-1}$ in a dispersion of height $9.2 \mathrm{~mm}$, the contact time $t=0.046 \mathrm{~s}$; these are the input parameters used by Springer et al. in the NEQ model implementation. ${ }^{23}$

For this contact time, the composition of the vapor bubble leaving the tray is

$$
y_{1}=0.06778, y_{2}=0.45057 \text {, and } y_{3}=0.48165 \text {. }
$$

This vapor composition is on the other side of the distillation boundary. Such boundary crossing is observed in Run T3-23 of the experiments of Springer et al. ${ }^{21,23,25,26}$ (cf. Figure 33). The component efficiency of water is higher than that of partner species; see Figure 39b. Water is the least volatile of the three components, and its transfer is directed from vapor to the liquid phase; a higher efficiency of water ensures that the liquid phase is richer in water than anticipated on the basis of equal component efficiencies.

The time-averaged mass transfer coefficient for the dispersed bubbles, for contact time $t=0.046 \mathrm{~s}$ is

$$
\left[k_{d}\right]=-\ln [Q] \frac{d_{\text {bubble }}}{6 t}
$$

For $t=0.046 \mathrm{~s}$, we have the matrix describing the departure from equilibrium is

$$
[Q]=\left[\begin{array}{cc}
0.12238 & 2.43476 \times 10^{-3} \\
0.10287 & 0.2959
\end{array}\right]
$$

and 


$$
\left[k_{d}\right]=\left[\begin{array}{cc}
343.22267 & -2.0244 \\
-85.52786 & 198.94608
\end{array}\right] \times 10^{-4}
$$

If the diffusion coupling effects are completely ignored, and the simplest Fickian relation

$$
J_{i}=-c_{t} D \frac{d x_{i}}{d z} ; \quad i=1,2,3
$$

is employed, the equilibration trajectory will be linear, running parallel to the residue curves (pink lines) in Figure 39a.

Let us examine what influence inclusion of liquid phase mass transfer resistance has on the composition trajectories.

At the liquid composition on the tray: $x_{1}=0.095, x_{2}=0.6345$, and $x_{3}=0.2705$, we calculate the following values of the M-S diffusivities of the binary pairs

$$
\bigoplus_{12}=6.06 ; \quad \bigoplus_{13}=4.95 ; \quad \bigoplus_{23}=3.1 \times 10^{-9} \mathrm{~m}^{2} \mathrm{~s}^{-1}
$$

For estimation of the Fick diffusivity, we use the approximation ${ }^{6}$

$$
[D]=|\Lambda|^{1 / 2}[\Gamma]
$$

The value of the scalar diffusivity is

$$
|\Lambda|^{1 / 2}=\sqrt{\frac{\bigoplus_{12} \bigoplus_{13} \Theta_{23}}{x_{1} \bigoplus_{23}+x_{2} \bigoplus_{13}+x_{3} \bigoplus_{12}}}=4.28 \times 10^{-9} \mathrm{~m}^{2} \mathrm{~s}^{-1}
$$

The matrix of thermodynamic factors is

$$
[\Gamma]=\left[\begin{array}{cc}
0.81047 & -0.06719 \\
-0.27874 & 0.67141
\end{array}\right]
$$

The Fick diffusivity matrix in the liquid phase is calculated as

$$
[D]=|\Lambda|^{1 / 2}[\Gamma]=\left[\begin{array}{cc}
3.46945 & -0.28763 \\
-1.19322 & 2.874181
\end{array}\right] \times 10^{-9}
$$

The mass transfer coefficient external to the bubble can be estimated from the surface renewal theory $^{15,16}$ 
$\left[k_{c}\right]=2 \sqrt{\frac{[D]}{\pi t_{\text {renewal }}}}$

The characteristic time for surface renewal can be estimated is $t_{\text {renewal }}=d_{\text {bubble }} / V_{\text {bubble }}=0.0225 \mathrm{~s}$; this value is lower than the time the bubble takes to rise through the dispersion, $0.046 \mathrm{~s}$.

For a surface renewal time of $0.0225 \mathrm{~s}$, the mass transfer in the continuous liquid phase can be estimated from the surface renewal theory

$$
\left[k_{c}\right]=2 \sqrt{\frac{[D]}{\pi t_{\text {renewal }}}}=\left[\begin{array}{cc}
4.41342 & -0.19315 \\
-0.80125 & 4.0137
\end{array}\right] \times 10^{-4}
$$

The overall mass transfer coefficient can be calculated from the addition of resistances formula

$$
\left[K_{O d}\right]^{-1}=\left[k_{d}\right]^{-1}+\frac{c_{t V, d}}{c_{t L, c}}\left[\begin{array}{cc}
\frac{y_{1, e q}}{x_{1}} & 0 \\
0 & \frac{y_{2, e q}}{x_{2}}
\end{array}\right]\left[k_{c}\right]^{-1}
$$

Inserting the values of the partial mass transfer coefficients, we have for the overall mass transfer coefficients

$$
\begin{aligned}
{\left[K_{\text {Od }}\right]^{-1}=\left[\begin{array}{cc}
343.22267 & -2.0244 \\
-85.52786 & 198.94608
\end{array}\right]^{-1} \times 10^{4}+} \\
\frac{36.16254}{1.45983 \times 10^{4}}\left[\begin{array}{cc}
\frac{0.06324}{0.095} & 0 \\
0 & \frac{0.36863}{0.6345}
\end{array}\right]\left[\begin{array}{cc}
4.41342 & -0.19315 \\
-0.80125 & 4.0137
\end{array}\right]^{-1} \times 10^{4}
\end{aligned}
$$

The matrix of overall mass transfer coefficients is

$$
\left[K_{\text {Od }}\right]=\left[\begin{array}{cc}
304.303387 & -2.69663 \\
-74.45095 & 185.81078
\end{array}\right] \times 10^{-4} \text {. }
$$

We note that $\left[K_{O d}\right] \approx\left[k_{d}\right]$, indicating that the mass transfer is controlled by diffusion within the vapor bubbles; the liquid phase mass transfer resistance is of negligible importance.

The matrix for the overall number of transfer units can be calculated from

$$
\left[N T U_{O d}\right]=\left[K_{O d}\right] a t
$$

The interfacial area per unit volume of bubble is 
$a=\frac{6}{d_{\text {bubble }}}=1.333 \times 10^{3}$

Therefore, for contact time $t=0.046 \mathrm{~s}$ is

$\left[N T U_{O \mathrm{~d}}\right]=\left[\begin{array}{cc}1.86639 & -0.01654 \\ -0.45663 & 1.13964\end{array}\right]$

The matrix describing departure from equilibrium can be determined from

$[Q]=\exp \left[-\left[N T U_{O d}\right]\right]$

$[Q]=\left[\begin{array}{cc}0.15544 & 3.76551 \times 10^{-3} \\ 0.10396 & 0.3209\end{array}\right]$

The composition of the vapor bubble exiting the tray at $t=0.046 \mathrm{~s}$ is determined from $\left(y-x_{e q}\right)=[Q]\left(y_{0}-x_{e q}\right)$

The calculated value is

$y_{1}=0.06918, y_{2}=0.45725$, and $y_{3}=0.47357$.

This vapor composition is very close to the value calculated above by ignoring the liquid phase resistance. Boundary crossing persists even if we ignore the liquid phase mass transfer resistance. The Geddes provides an accurate prediction of boundary crossing for the system water(1)/ethanol(2)/methylacetate(3).

For various vapor compositions entering any given stage, we have plotted in Figure 39c the actual composition vector $\left(y_{i, L}-y_{i, E}\right)$, calculated from the NEQ model (taking bubble diameter of $4.5 \mathrm{~mm}$ ) along with the equilibrium vector $\left(y_{i}^{*}-y_{i, E}\right)$. The angle between the NEQ trajectory (red line) and the EQ trajectory (blue line) increases when the differences in the component efficiencies increase. If all the component efficiencies were equal to one another, the NEQ and EQ trajectories would coincide. We see from Figure 39c that the NEQ trajectory has a tendency to cut across to the right of the EQ trajectory, precisely as has been observed in Run T3-23; cf. Figure 33. It is this tendency to cut towards the right of the composition space that causes boundary crossing. By performing several NEQ 
simulations with various starting compositions of the vapor entering the condenser we can determine the region within which the column trajectories will cross the distillation boundary and end up with reboiler compositions in the right region. This boundary crossing region is shown as the orange shaded area in Figure 39d.

\section{Boundary crossing in heterogeneous azeotropic distillation}

For heterogeneous azeotropic distillation, experimental data on column composition trajectories for water/acetone/toluene, and water/ethanol/cyclohexane show boundary crossing characteristics that are attributable to diffusional coupling. ${ }^{22}$

The experimental data for Run WAT-1, and WAT-2 for water(1)/acetone(2)/toluene(3) mixtures are shown in Figure 40, and Figure 41. The NEQ model simulations, taking due account of coupling in both vapor and liquid phases are indicated by the continuous red lines. The EQ model simulations (i.e. ignoring diffusional coupling, and assuming that the efficiencies of all components are the same) are indicated by the dashed red lines.

For the heterogeneous azeotropic distillation with water(1)/ethanol(2)/cyclohexane(3) mixture, boundary crossing is observed in Run WEC-12; see Figure 42. For Run WEC-8, no boundary crossing is observed; see Figure 43.

For rationalization and quantitative description of the observed experimental boundary crossing phenomena, Springer et al. ${ }^{22}$ used rigorous non-equilibrium (NEQ) stage-wise contacting model, as implemented in ChemSep. ${ }^{18,30}$ The NEQ model uses the Maxwell-Stefan formulation for diffusion in the vapor and the two liquid phases. There are four mass transfer resistances to contend with, as sketched in Figure 44; Springer et al. provide the modelling details. ${ }^{22}$

The important conclusion reached in their work is that boundary crossing effects are primarily attributable to diffusional coupling effects, that cause the component Murphree efficiencies to be unequal to one another. Unequal component efficiencies cause column composition trajectories to deviate from those of the residue curve maps. Put another way, the NEQ model does not follow the tramline guides of the RCM. 


\section{Separating azeotropic alcohol/water mixture by diffusional distillation}

If we distill a 2-propanol(1)/water (2) mixture of azeotropic composition in say a sieve tray column, the compositions of the vapor and liquid phases will remain unchanged as the vapor bubbles traverse along the height of the dispersion. The situation is changed if the distillation is carried out in the presence of an inert gas, say nitrogen (3); cf. Figure 45. Separation of the azeotropic mixture is attained because of diffusional coupling effects. For quantitative demonstration of this separation, let us consider a sieve tray column operating at a total pressure of $101.3 \mathrm{kPa}$ and temperature of $313.15 \mathrm{~K}$. The liquid composition entering the tray is $x_{1}=0.6226, x_{2}=0.3774$; this composition corresponds to that of the azeotrope at $313.15 \mathrm{~K}$, calculated using the NRTL parameters provided in Table 19.

We bubble pure nitrogen (3) through the tray, i.e. the vapor composition entering the tray is $y_{1 \mathrm{E}}=0.0$, $y_{2 \mathrm{E}}=0.0, y_{3 \mathrm{E}}=1.0$. If the vapor bubble is allowed to equilibrate, the composition of the equilibrated vapor is $y_{1, \mathrm{eq}}=0.09836, y_{2, \mathrm{eq}}=0.05963, y_{3 \mathrm{E}}=0.84201$. The ratio of the compositions of water (2) to that of 2-propanol (1) at equilibrium, $\frac{y_{2, e q}}{y_{1, e q}}$ is that same as that of the ratio of water to 2-propanol in the liquid phase, $\frac{y_{2, e q}}{y_{1, e q}}=\frac{x_{2}}{x_{1}}=0.606$.

Let us consider the dispersion to consist of uniform and rigid vapor bubbles of diameter, $d_{\text {bubble }}$. The transient equilibration process within a rigid spherical bubble is described by Geddes model that was originally developed for describing binary diffusion inside vapor bubbles on distillation trays. ${ }^{7}$ For ternary mixtures, the Geddes model can be written in two-dimensional matrix differential equation ${ }^{4,31}$

$$
\left(y-x_{e q}\right)=[Q]\left(y_{0}-x_{e q}\right) ; \quad[Q] \equiv \frac{6}{\pi^{2}} \sum_{m=1}^{\infty} \frac{1}{m^{2}} \exp \left[-m^{2} \pi^{2} \frac{4[D] t}{d_{b u b b l e}^{2}}\right]
$$

In the above equation, $\left(y_{0}\right)$ denotes the vapor composition entering the tray. The Sylvester theorem, detailed in Appendix A of Taylor and Krishna, ${ }^{4}$ is required for explicit calculation of the composition trajectories described by the Geddes model. For vapor bubbles rising on a sieve tray, the effective 
contact time of the dispersed phase bubbles with the surrounding continuous phase is $t=h_{f} / V_{\text {bubble }}$, where $h_{\mathrm{f}}$ is the froth dispersion height, and $V_{\text {bubble }}$ is the bubble rise velocity.

We choose a bubble diameter, $d_{\text {bubble }}=4.5 \mathrm{~mm}$, rising at a velocity $V_{\text {buble }}=0.2 \mathrm{~m} \mathrm{~s}^{-1}$ through a froth dispersion $h_{f}$ of height $9.2 \mathrm{~m}$. These parameters are representative of the sieve-tray experimental set of Springer et al. ${ }^{21,23,25,26}$

In Figure 46a, the mole fractions of 2-propanol (1) and water (2) are plotted as a function of time, $t$. The ratio of the mole fraction of water (2) to that of 2-propanol (1) in the vapor phase as a function of time, $t$ is plotted in Figure 46a; this ratio equilibrates to the value of $\frac{y_{2, e q}}{y_{1, e q}}=\frac{x_{2}}{x_{1}}=0.606$, as is expected. During the initial transience, however, the vapor is richer in water vapor than 2-propanol. For the contact time of the vapor bubbles, $t=h_{f} / V_{\text {bubble }}=0.46 \mathrm{~s}$, the ratio of the mole fraction of water (2) to that of 2-propanol (1) is 0.738 ; this value is significantly higher than that of the azeotrope as seen in Figure 46b. This implies that the 2-propanol/water azeotrope can be separated by bubbling nitrogen through the tray.

In order to understand the mechanism of the separation, let us examine the diffusivities and fluxes in the ternary mixture. The values of the vapor phase M-S diffusivities of the three binary pairs at 313.15 K, calculated using the Fuller-Schettler-Giddings ${ }^{32}$ method, are $\bigoplus_{12}=1.47 ; \quad \bigoplus_{13}=1.15 ; \quad \bigoplus_{23}=2.81 \times 10^{-5} \mathrm{~m}^{2} \mathrm{~s}^{-1} ;$ these diffusivities are independent of composition. At the average composition between the entering vapor compositions and the equilibrated compositions, the Fick matrix of diffusivities $[D]=\left[\begin{array}{cc}1.186 & -0.15399 \\ 0.159 & 2.16759\end{array}\right] \times 10^{-5} \mathrm{~m}^{2} \mathrm{~s}^{-1}$. Water vapor is the smallest of the three molecules and the mobility of water molecules in the vapor phase is the highest. This is evident because of the significantly larger value of $D_{22}$ as compared to $D_{11}$. The driving forces are $\Delta y_{1}=y_{10}-y_{1, e q}=-0.09859$, and $\Delta y_{2}=y_{1 o}-y_{2, e q}=-0.05615$. Both driving forces are negative, i.e. directed from liquid to the vapor phase. The ratio of the flux of water to that of 2-propanol is 
$\frac{D_{21} \Delta y_{1}+D_{22} \Delta y_{2}}{D_{11} \Delta y_{1}+D_{12} \Delta y_{2}}=1.35$. This ratio is higher than that corresponding to the azeotropic composition. Taking account of coupling effects results in a higher proportion of water vapor in the bubble during equilibration. Coupling effects enhance the flux of water, relative to that of 2-propanol, due to two reasons. Firstly, the contribution of $D_{21} \Delta y_{1}$ has the same sign as that of $D_{22} \Delta y_{2}$; therefore flux of water is enhanced. Secondly, the contribution of $D_{12} \Delta y_{2}$ reduces the flux of 2-propanol because $D_{12}$ is negative in sign.

The experiments of Fullarton and Schlunder ${ }^{34}$ confirm that the concept of diffusion distillation is effective for separation of 2-propanol/water azeotrope.

If helium is used as inert gas in place of nitrogen, the separations are less effective; see the comparisons of the ratio of water to 2-propanol in the vapor phase presented in Figure 46c. With helium, the ratio of the flux of water to that of 2-propanol is $\frac{D_{21} \Delta y_{1}+D_{22} \Delta y_{2}}{D_{11} \Delta y_{1}+D_{12} \Delta y_{2}}=0.85$; this ratio is lower than that with nitrogen as inert gas. Use of argon as inert gas improves the separations because in this case the ratio of the flux of water to that of 2-propanol is $\frac{D_{21} \Delta y_{1}+D_{22} \Delta y_{2}}{D_{11} \Delta y_{1}+D_{12} \Delta y_{2}}=1.46$.

Figure 46d compares the diffusion equilibration trajectories in composition space. Coupling effects are strongest with argon and this explains why the equilibration trajectories deviates the most from the linear equilibration that results with uncoupled diffusion.

Diffusional distillation can be also used to separate ethanol/water mixture of azeotropic composition. For quantitative demonstration of this separation, let us consider a sieve tray column operating at a total pressure of $101.3 \mathrm{kPa}$ and temperature of $313.15 \mathrm{~K}$. The liquid composition entering the tray is $x_{1}=$ $0.87013, x_{2}=0.12987$; this composition corresponds to that of the azeotrope at $313.15 \mathrm{~K}$, calculated using the NRTL parameters provided in Table 20.

We bubble pure nitrogen (3) through the tray, i.e. the vapor composition entering the tray is $y_{1 \mathrm{E}}=0.0$, $y_{2 \mathrm{E}}=0.0, y_{3 \mathrm{E}}=1.0$. If the vapor is allowed to equilibrate, the composition of the equilibrated vapor is $y_{1, \text { eq }}=0.15266, y_{2, \text { eq }}=0.02279, y_{3 \mathrm{E}}=0.82455$. The ratio of the compositions of water (2) to that of 
ethanol (1) at equilibrium, $\frac{y_{2, e q}}{y_{1, e q}}$ is that same as that of the ratio of water to ethanol in the liquid phase, $\frac{y_{2, e q}}{y_{1, e q}}=\frac{x_{2}}{x_{1}}=0.14925$

In Figure 47a, the mole fractions of ethanol (1) and water (2) are plotted as a function of time, $t$. The ratio of the mole fraction of water (2) to that of ethanol (1) in the vapor phase as a function of time, $t$ is plotted in Figure 47b; this ratio equilibrates to the value of $\frac{y_{2, e q}}{y_{1, e q}}=\frac{x_{2}}{x_{1}}=0.14925$, as is expected. During the initial transience, however, the vapor is richer in water vapor than ethanol. For the contact time of the vapor bubbles, $t=h_{f} / V_{\text {bubble }}=0.46 \mathrm{~s}$, the ratio of the mole fraction of water (2) to that of ethanol (1) is 0.1726 ; this value is higher than that of the azeotrope as seen in Figure 47b. This implies that the ethanol/water azeotrope can be separated by bubbling nitrogen through the tray.

In order to understand the mechanism of the separation, let us examine the diffusivities and fluxes in the ternary mixture. The values of the vapor phase M-S diffusivities of the three binary pairs at 313.15 K, calculated using the Fuller-Schettler-Giddings ${ }^{32}$ method, are $\bigoplus_{12}=1.75 ; \quad \bigoplus_{13}=1.37 ; \quad \bigoplus_{23}=2.81 \times 10^{-5} \mathrm{~m}^{2} \mathrm{~s}^{-1} ;$ these diffusivities are independent of composition. At the average composition between the entering vapor compositions and the equilibrated compositions, the Fick matrix of diffusivities $[D]=\left[\begin{array}{cc}1.38892 & -0.20907 \\ 0.04335 & 2.21543\end{array}\right] \times 10^{-5} \mathrm{~m}^{2} \mathrm{~s}^{-1}$. Water vapor is the smallest of the three molecules and the mobility of water molecules in the vapor phase is the highest. This is evident because of the significantly larger value of $D_{22}$ as compared to $D_{11}$. The driving forces are $\Delta y_{1}=y_{10}-y_{1, e q}=-0.15266$, and $\Delta y_{2}=y_{1 o}-y_{2, e q}=-0.02278$. Both driving forces are negative, i.e. directed from liquid to the vapor phase. The ratio of the flux of water to that of ethanol is $\frac{D_{21} \Delta y_{1}+D_{22} \Delta y_{2}}{D_{11} \Delta y_{1}+D_{12} \Delta y_{2}}=0.27546$. This ratio is higher than that corresponding to the azeotropic composition. Taking account of coupling effects results in a higher proportion of water vapor in the bubble during 
equilibration. Coupling effects enhance the flux of water, relative to that of ethanol, due to two reasons. Firstly, the contribution of $D_{21} \Delta y_{1}$ has the same sign as that of $D_{22} \Delta y_{2}$; therefore flux of water is enhanced. Secondly, the contribution of $D_{12} \Delta y_{2}$ reduces the flux of ethanol because $D_{12}$ is negative in sign.

If helium is used as inert gas in place of nitrogen, the separations are less effective; see the comparisons of the ratio of water to ethanol in the vapor phase presented in Figure 47c. With helium, the ratio of the flux of water to that of ethanol is $\frac{D_{21} \Delta y_{1}+D_{22} \Delta y_{2}}{D_{11} \Delta y_{1}+D_{12} \Delta y_{2}}=0.197$; this ratio is lower than that with nitrogen as inert gas. Use of argon as inert gas improves the separation of the azeotrope because the ratio of the flux of water to that of ethanol is $\frac{D_{21} \Delta y_{1}+D_{22} \Delta y_{2}}{D_{11} \Delta y_{1}+D_{12} \Delta y_{2}}=0.291$

Figure $47 \mathrm{~d}$ compares the diffusion equilibration trajectories in composition space. Coupling effects are strongest with argon and this explains why the equilibration trajectories deviates the most from the linear equilibration that results with uncoupled diffusion.

Experimental confirmation of the validity of the diffusional distillation concept to separate ethanol/water azeotrope is provided by Singh and Prasad. ${ }^{35}$ Of particular interest is their experimental observation that use of helium as inert gas yields lower separation selectivity as compared to nitrogen. Singh and Prasad ${ }^{35}$ also report that use of argon as inert gas improves the separation selectivity.

The technique of bubbling inert gas to break azeotropes also applies to non-aqueous mixtures such as acetone/methanol. For quantitative demonstration of this separation, let us consider a sieve tray column operating at a total pressure of $101.3 \mathrm{kPa}$ and temperature of $313.15 \mathrm{~K}$. The liquid composition entering the tray is $x_{1}=0.85259, x_{2}=0.14741$; this composition corresponds to that of the azeotrope at $313.15 \mathrm{~K}$, calculated using the NRTL parameters provided in Table 21

We bubble pure nitrogen (3) through the tray, i.e. the vapor composition entering the tray is $y_{1 \mathrm{E}}=0.0$, $y_{2 \mathrm{E}}=0.0, y_{3 \mathrm{E}}=1.0$. If the vapor is allowed to equilibrate, the composition of the equilibrated vapor is $y_{1, \mathrm{eq}}=0.4798, y_{2, \mathrm{eq}}=0.08296, y_{3 \mathrm{E}}=0.43724$. The ratio of the compositions of methanol (2) to that of 
acetone (1) at equilibrium, $\frac{y_{2, e q}}{y_{1, e q}}$ is that same as that of the ratio of water to ethanol in the liquid phase, $\frac{y_{2, e q}}{y_{1, e q}}=\frac{x_{2}}{x_{1}}=0.1729$

In Figure 48a, the mole fractions of acetone (1) and methanol (2) are plotted as a function of time, $t$. The ratio of the mole fraction of methanol (2) to that of acetone (1) in the vapor phase as a function of time, $t$ is plotted in Figure 48b; this ratio equilibrates to the value of $\frac{y_{2, e q}}{y_{1, e q}}=\frac{x_{2}}{x_{1}}=0.129$, as is expected. During the initial transience, however, the vapor is richer in water vapor than ethanol. For the contact time of the vapor bubbles, $t=h_{f} / V_{\text {bubble }}=0.46 \mathrm{~s}$, the ratio of the mole fraction of methanol (2) to that of acetone (1) is 0.1908 ; this value is higher than that of the azeotrope as seen in Figure $48 \mathrm{~b}$. This implies that the acetone/methanol azeotrope can be separated by bubbling nitrogen through the tray.

In order to understand the mechanism of the separation, let us examine the diffusivities and fluxes in the ternary mixture. The values of the vapor phase M-S diffusivities of the three binary pairs at 313.15 K, calculated using the Fuller-Schettler-Giddings ${ }^{32}$ method, are $\bigoplus_{12}=0.98 ; \quad \bigoplus_{13}=1.186 ; \bigoplus_{23}=1.79 \times 10^{-5} \mathrm{~m}^{2} \mathrm{~s}^{-1} ;$ these diffusivities are independent of composition. At the average composition between the entering vapor compositions and the equilibrated compositions, the Fick matrix of diffusivities $[D]=\left[\begin{array}{cc}1.17224 & 0.11647 \\ 0.05285 & 1.3305\end{array}\right] \times 10^{-5} \mathrm{~m}^{2} \mathrm{~s}^{-1}$. The driving forces are $\Delta y_{1}=y_{10}-y_{1, e q}=-0.4798$, and $\Delta y_{2}=y_{1 o}-y_{2, e q}=-0.08296$. Both driving forces are negative, i.e. directed from liquid to the vapor phase. The ratio of the flux of methanol to that of acetone is $\frac{D_{21} \Delta y_{1}+D_{22} \Delta y_{2}}{D_{11} \Delta y_{1}+D_{12} \Delta y_{2}}=0.23725$. This ratio is higher than that corresponding to the azeotropic composition. Taking account of coupling effects results in a higher proportion of methanol vapor in the bubble during equilibration. 
If helium is used as inert gas in place of nitrogen, the separations are less effective; see the comparisons of the ratio of methanol to acetone in the vapor phase presented in Figure 48c. With helium, the ratio of the flux of water to that of ethanol is $\frac{D_{21} \Delta y_{1}+D_{22} \Delta y_{2}}{D_{11} \Delta y_{1}+D_{12} \Delta y_{2}}=0.19682 ;$ this ratio is lower than that with nitrogen as inert gas. Use of argon as inert gas improves the separation of the azeotrope because the ratio of the flux of water to that of ethanol is $\frac{D_{21} \Delta y_{1}+D_{22} \Delta y_{2}}{D_{11} \Delta y_{1}+D_{12} \Delta y_{2}}=0.24574$.

Figure 48d compares the diffusion equilibration trajectories in composition space. Coupling effects are strongest with argon and this explains why the equilibration trajectories deviates the most from the linear equilibration that results with uncoupled diffusion. 


\section{Notation}

$A_{\mathrm{m}} \quad$ constant in Kronig-Brink model, dimensionless

$c_{\mathrm{t}} \quad$ total molar concentration of mixture, $\mathrm{mol} \mathrm{m}^{-3}$

$d_{\text {bubble }} \quad$ bubble diameter, $\mathrm{m}$

$d_{\text {drop }} \quad$ droplet diameter, $\mathrm{m}$

$\bigoplus_{\mathrm{ij}} \quad$ M-S binary pair diffusivity, $\mathrm{m}^{2} \mathrm{~s}^{-1}$

$[D] \quad$ Fick diffusivity matrix, $\mathrm{m}^{2} \mathrm{~s}^{-1}$

$|D| \quad$ Determinant of the Fick diffusivity matrix, $\mathrm{m}^{4} \mathrm{~s}^{-2}$

$|D|^{1 / 2} \quad$ Square-root of determinant of $[D], \mathrm{m}^{2} \mathrm{~s}^{-1}$

$E_{\mathrm{i}} \quad$ Component Murphree efficiency, dimensionless

Fo $\quad$ Fourier number, dimensionless

[I] Identity matrix, dimensionless

$J_{\mathrm{i}} \quad$ molar diffusion flux of species $i$ with respect to $u, \mathrm{~mol} \mathrm{~m}^{-2} \mathrm{~s}^{-1}$

[k] Matrix of mass transfer coefficients, $\mathrm{m} \mathrm{s}^{-1}$

$[K] \quad$ Matrix of overall mass transfer coefficients, $\mathrm{m} \mathrm{s}^{-1}$

[NTU] Matrix of number of transfer units, dimensionless

$p \quad$ system pressure, $\mathrm{Pa}$

$[Q] \quad$ matrix quantifying fractional departure from equilibrium, dimensionless

$R \quad$ gas constant, $8.314 \mathrm{~J} \mathrm{~mol}^{-1} \mathrm{~K}^{-1}$

$t \quad$ time, $\mathrm{s}$

$T \quad$ absolute temperature, $\mathrm{K}$

$x_{\mathrm{i}} \quad$ mole fraction of component $i$ in liquid phase, dimensionless

$y_{\mathrm{i}} \quad$ mole fraction of component $i$ in vapor phase, dimensionless

$u \quad$ molar average mixture velocity, $\mathrm{m} \mathrm{s}^{-1}$

$V_{\text {bubble }} \quad$ bubble rise velocity, $\mathrm{m} \mathrm{s}^{-1}$

$V_{d r o p} \quad$ droplet rise velocity, $\mathrm{m} \mathrm{s}^{-1}$ 


\section{Greek letters}

$\beta \quad$ constant used for equilibration in Lewis stirred cell, $\mathrm{m}^{-2}$

$\delta_{\mathrm{ij}} \quad$ Kronecker delta, dimensionless

$\gamma_{\mathrm{i}} \quad$ activity coefficient of component $i$, dimensionless

$[\Gamma]$ matrix of thermodynamic factors, dimensionless

$|\Gamma|^{1 / 2} \quad$ Square-root of determinant of $[\Gamma]$, dimensionless

$\lambda_{\mathrm{m}} \quad$ constant in Kronig-Brink model, dimensionless

$[\Lambda] \quad$ matrix defined by Equation (4), $\mathrm{m}^{2} \mathrm{~s}^{-1}$

$|\Lambda|^{1 / 2} \quad$ Square-root of determinant of $[\Lambda], \mathrm{m}^{2} \mathrm{~s}^{-1}$

$\mu_{\mathrm{i}} \quad$ molar chemical potential, $\mathrm{J} \mathrm{mol}^{-1}$

\section{Subscript}

0

Referring to starting compositions, $t=0$

bubble Referring to bubble

c

Referring to continuous phase

d Referring to dispersed phase

drop Referring to droplet

eq Referring to final equilibrated compositions, $t \rightarrow \infty$

E Referring to vapor compositions entering tray

i Component number

j Component number

L referring to liquid phase

O referring to overall parameter

L referring to vapor phase 
Table 1. NRTL parameters for heptane(1)/toluene(2)/sulpholane(3). The parameters are taken from Table 6.8 of the $\mathrm{PhD}$ dissertation of Meindersma. ${ }^{36}$

\begin{tabular}{|l|l|l|l|}
\hline & $\tau_{i j}$ & $\tau_{j i}$ & $\alpha_{i j}=\alpha_{j i}$ \\
\cline { 2 - 4 } & dimensionless & dimensionless & dimensionless \\
\hline $\begin{array}{l}\text { heptane(1)/ } \\
\text { toluene(2) }\end{array}$ & $-0.756+48.1 / T$ & $0.269+31.74 / T$ & 0.3 \\
\hline $\begin{array}{l}\text { heptane(1)/ } \\
\text { sulpholane(3) }\end{array}$ & $-0.039+1365 / T$ & $-0.215+1188 / T$ & 0.3 \\
\hline $\begin{array}{l}\text { toluene(2)/ } \\
\text { sulpholane(3) }\end{array}$ & $-1.057+1017 / T$ & $0.428-165.7 / T$ & 0.3 \\
\hline
\end{tabular}

Table 2. The values of $A_{m}$ and $\lambda_{m}$ are tabulated by Sideman and Shabtai. ${ }^{14}$

\begin{tabular}{|l|l|l|}
\hline Integer counter, $m$ & $A_{m}$ & $\lambda_{m}$ \\
\hline 1 & 1.29 & 1.656 \\
\hline 2 & 0.596 & 9.08 \\
\hline 3 & 0.386 & 22.2 \\
\hline 4 & 0.35 & 36.5 \\
\hline 5 & 0.28 & 63 \\
\hline 6 & 0.22 & 89.8 \\
\hline 7 & 0.16 & 123.8 \\
\hline
\end{tabular}


Table 3. NRTL parameters for NMP(1)/propylbenzene(2)/tetradecane(3) at 298.15 K. The parameters are from Al-Jimaz et al. ${ }^{37}$

\begin{tabular}{|l|l|l|l|}
\hline \multirow{2}{*}{} & $\tau_{i j}=A_{i j} / T$ & $\tau_{j i}=A_{j i} / T$ & $\alpha_{i j}=\alpha_{j i}$ \\
\cline { 2 - 4 } & dimensionless & dimensionless & dimensionless \\
\hline NMP(1)/ & -2.8661 & 1.5931 & 0.2 \\
\hline propylbenzene(2) & & & 0.2 \\
\hline $\begin{array}{l}\text { propylbenzene(2)/ } \\
\text { tetradecane(3) }\end{array}$ & 0.2851 & 0.4103 & 0.2 \\
\hline
\end{tabular}

Table 4. NRTL parameters for glyercol(1)/acetone(2)/water(3) at $298 \mathrm{~K}$. These parameters are from Krishna et al. $^{5}$

\begin{tabular}{|l|l|l|l|}
\hline & $\tau_{i j}=A_{i j} / T$ & $\tau_{j i}=A_{j i} / T$ & $\alpha_{i j}=\alpha_{j i}$ \\
\cline { 2 - 4 } & dimensionless & dimensionless & dimensionless \\
\hline glycerol(1)/acetone(2) & 0.868 & 2.467 & 0.2 \\
\hline glycerol(1)/water(3) & -1.29 & -1.52 & 0.2 \\
\hline acetone(2)/water(3) & -0.665 & 2.095 & 0.2 \\
\hline
\end{tabular}


Table 5. NRTL parameters for water(1)/acetone(2)/phenol(3) at 323.15 K. These parameters are taken from Table 2 of Zuber et al. ${ }^{38}$

\begin{tabular}{|l|l|l|l|}
\hline & $\tau_{i j}=A_{i j} / T$ & $\tau_{j i}=A_{j i} / T$ & $\alpha_{i j}=\alpha_{j i}$ \\
\cline { 2 - 4 } & dimensionless & dimensionless & dimensionless \\
\hline water(1)/acetone(2) & 0.1024 & 1.826 & 0.2 \\
\hline water(1)/phenol(3) & 5.332 & -1.585 & 0.2 \\
\hline acetone(2)/phenol(3) & -1.504 & -2.78 & 0.2 \\
\hline
\end{tabular}

Table 6. UNIQUAC parameters for acetone(1)/ethyl-acetate(2)/water(3) at $293 \mathrm{~K}$. These parameters are from Pertler. ${ }^{39}$

\begin{tabular}{|l|l|l|}
\hline \multirow{2}{*}{} & $r_{i}$ & $q_{i}$ \\
\cline { 2 - 3 } & dimensionless & dimensionless \\
\hline acetone(1) & 2.5735 & 2.336 \\
\hline ethyl-acetate(2) & 3.4786 & 3.116 \\
\hline water(3) & 0.92 & 1.4 \\
\hline
\end{tabular}

\begin{tabular}{|l|l|l|}
\hline \multirow{2}{*}{ acetone(1)/ethyl-acetate(2) } & $\tau_{i j}=\exp \left(-A_{i j} / T\right)$ & $\tau_{j i}=\exp \left(-A_{j i} / T\right)$ \\
\cline { 2 - 3 } & dimensionless & dimensionless \\
\hline acetone(1)/water(3) & 1.3068 & 0.827 \\
\hline ethyl-acetate(2)/water(3) & 0.488 & 1.328 \\
\hline
\end{tabular}


Table 7. UNIQUAC parameters for water(1)/caprolactam(2)/toluene(3) at 298.15 K. These parameters are from Table 1, Chapter 7 of the $\mathrm{PhD}$ dissertation of Bollen. ${ }^{40}$

\begin{tabular}{|l|l|l|}
\hline \multirow{2}{*}{} & $r_{i}$ & $q_{i}$ \\
\cline { 2 - 3 } & dimensionless & dimensionless \\
\hline water(1) & 0.92 & 1.4 \\
\hline caprolactam(2) & 4.6106 & 3.724 \\
\hline toluene(3) & 3.9928 & 2.968 \\
\hline
\end{tabular}

\begin{tabular}{|l|l|l|}
\hline \multirow{2}{*}{ water(1)/caprolactam(2) } & $\tau_{i j}=A_{i j} / T$ & $\tau_{j i}=A_{j i} / T$ \\
\cline { 2 - 3 } & dimensionless & dimensionless \\
\hline water(1)/ toluene(3) & 0.1027043 & 3.647516849 \\
\hline caprolactam(2)/toluene(3) & 0.2563201 & 0.0964476 \\
\hline
\end{tabular}


Table 8. NRTL parameters for water(1)/acetic acid(2)/isophorone(3) at 298.15 K. The parameters are from Colombo et al. ${ }^{41}$

\begin{tabular}{|l|l|l|l|}
\hline & $\tau_{i j}=A_{i j} / T$ & $\tau_{j i}=A_{j i} / T$ & $\alpha_{i j}=\alpha_{j i}$ \\
\cline { 2 - 4 } & dimensionless & dimensionless & dimensionless \\
\hline water(1)/acetic acid (2) & 0.7074 & 0.2455 & 0.2 \\
\hline water(1)/isophorone(3) & 6.466 & -0.2852 & 0.2 \\
\hline $\begin{array}{l}\text { Acetic acid(2)/ } \\
\text { Isophorone(3) }\end{array}$ & -1.489 & 1.381 & 0.2 \\
\hline
\end{tabular}

Table 9. NRTL parameters for water(1)/acetic acid(2)/MTBE (3) at 298.15 K. The parameters are from Zhang and Wang. ${ }^{42}$

\begin{tabular}{|l|l|l|l|}
\hline & $\tau_{i j}=A_{i j} / T$ & $\tau_{j i}=A_{j i} / T$ & $\alpha_{i j}=\alpha_{j i}$ \\
\cline { 2 - 4 } & dimensionless & dimensionless & dimensionless \\
\hline water(1)/acetic acid (2) & 0.354 & -1.2151 & 0.47 \\
\hline water(1)/MTBE(3) & 3.9737 & 1.2998 & 0.2 \\
\hline $\begin{array}{l}\text { Acetic acid(2)/ } \\
\text { MTBE(3) }\end{array}$ & -0.2774 & -2.8068 & 0.37 \\
\hline
\end{tabular}


Table 10. NRTL parameters for $[\mathrm{omim}][\mathrm{Cl}](1) /$ ethanol(2)/TAEE(3) at $298 \mathrm{~K}$. The parameters are from Aznar. $^{43}$

\begin{tabular}{|l|l|l|l|}
\hline \multirow{2}{*}{} & $\tau_{i j}=A_{i j} / T$ & $\tau_{j i}=A_{j i} / T$ & $\alpha_{i j}=\alpha_{j i}$ \\
\cline { 2 - 4 } & dimensionless & dimensionless & dimensionless \\
\hline$[$ omim $][\mathrm{Cl}](1) /$ ethanol(2) & -1.674 & -3.035 & 0.2 \\
\hline$[$ omim] $[\mathrm{Cl}](1) /$ TAEE(3) & 1.365 & 9.3245 & 0.204 \\
\hline ethanol(2)/TAEE(3) & 0.3034 & 1.399 & 0.307 \\
\hline
\end{tabular}

Table 11. UNIQUAC parameters for [bmim][TfO](1)/ethanol(2)/TAEE(3) at $298 \mathrm{~K}$. The parameters are from Tables 2, 3, and 4 of Santiago et al. ${ }^{44}$

\begin{tabular}{|l|l|l|}
\hline \multirow{2}{*}{} & $r_{i}$ & $q_{i}$ \\
\cline { 2 - 3 } & dimensionless & dimensionless \\
\hline$[$ bmim][TfO] & 8.9463 & 7.135 \\
\hline ethanol(2) & 2.5755 & 2.588 \\
\hline TAEE(3) & 5.417 & 4.712 \\
\hline
\end{tabular}

\begin{tabular}{|l|l|l|}
\hline & $\tau_{i j}=\exp \left(-A_{i j} / T\right)$ & $\tau_{j i}=\exp \left(-A_{j i} / T\right)$ \\
\cline { 2 - 3 } & dimensionless & dimensionless \\
\hline$[\mathrm{bmim}][$ TfO](1)/ethanol(2) & 0.8331298 & 2.91830955 \\
\hline$[\mathrm{bmim}][$ TfO](1)/TAEE(3) & 1.4677043 & 0.004246414 \\
\hline ethanol(2)/TAEE(3) & 3.74432548 & 0.048064224 \\
\hline
\end{tabular}


Table 12. NRTL parameters for water(1)/ethanol(2)/cyclohexane (3). These parameters are from the DECHEMA Dortmund data bank, as reported in Table 1 of Springer et al. ${ }^{22}$ These parameters are used along with $G_{i j}=\exp \left(-\alpha_{i j} \tau_{i j}\right)$ and $\tau_{i j}=B_{i j} / T$.

\begin{tabular}{ll|ccc}
\hline Component $\mathrm{i}$ & Component $\mathrm{j}$ & $B_{\mathrm{ij}} / \mathrm{K}$ & $B_{\mathrm{ji}} / \mathrm{K}$ & $\alpha_{\mathrm{ij}}$ \\
\hline Water & Ethanol & 557.48 & 29.09 & 0.348 \\
Water & Cyclohexane & 4422.3 & 1688.3 & 0.212 \\
Ethanol & Cyclohexane & 440.61 & 717.68 & 0.463
\end{tabular}

Table 13. NRTL parameters for toluene(1)/ethanol(2)/water(3) at $298 \mathrm{~K}$. The toluene/water parameters are from Wang et al. ${ }^{45}$ The remaining parameters are from the DECHEMA Dortmund data bank; the ethanol/toluene $\tau_{j i}$ has been modified to match experimental binodal data. ${ }^{46,47}$

\begin{tabular}{|l|l|l|l|}
\hline & $\tau_{i j}$ & $\tau_{j i}$ & $\alpha_{i j}=\alpha_{j i}$ \\
\cline { 2 - 4 } & dimensionless & dimensionless & dimensionless \\
\hline toluene(1)/ethanol(2) & 1.938 & 0.6 & 0.529 \\
\hline toluene(1)/water(3) & 15.219 & 7.529 & 0.2 \\
\hline ethanol(2)/water(3) & -0.0978 & 2.096 & 0.293 \\
\hline
\end{tabular}


Table 14. NRTL parameters for water(1)/acetone(2)/toluene(3). These parameters are from the DECHEMA Dortmund data bank, as reported in Table 1 of Springer et al. ${ }^{22}$ These parameters are used along with $G_{i j}=\exp \left(-\alpha_{i j} \tau_{i j}\right)$ and $\tau_{i j}=B_{i j} / T$.

\begin{tabular}{ll|ccc}
\hline Component i & Component $\mathrm{j}$ & $B_{\mathrm{ij}} / \mathrm{K}$ & $B_{\mathrm{ji}} / \mathrm{K}$ & $\alpha_{\mathrm{ij}}$ \\
\hline Water & Acetone & 653.89 & 377.58 & 0.586 \\
Water & Toluene & 2160.8 & 2839.4 & 0.200 \\
Acetone & Toluene & -124.77 & 366.1 & 0.295 \\
\hline
\end{tabular}


Table 15. NRTL parameters used in homogeneous and heterogeneous distillation systems.

NRTL parameters from Dortmund data bank.

$G_{\mathrm{ij}}=\exp \left(-\alpha_{\mathrm{ij}} \tau_{\mathrm{ij}}\right)$ and $\tau_{\mathrm{ij}}=B_{\mathrm{ij}} / T$

\begin{tabular}{|c|c|c|c|c|}
\hline Component $i$ & Component $j$ & $B_{\mathrm{ij}} / \mathrm{K}$ & $B_{\mathrm{ji}} / \mathrm{K}$ & $\alpha_{\mathrm{ij}}$ \\
\hline \multicolumn{5}{|c|}{ water/ethanol/acetone } \\
\hline Water & Ethanol & 624.9174 & -29.169 & 0.2937 \\
\hline Water & Acetone & 602.6252 & 330.4768 & 0.5103 \\
\hline Ethanol & Acetone & 188.8983 & 22.83319 & 0.3006 \\
\hline \multicolumn{5}{|c|}{ water/methanol/isopropanol } \\
\hline Water & Methanol & 594.6299 & -182.6052 & 0.297 \\
\hline Water & Isopropanol & 729.2208 & 70.6619 & 0.288 \\
\hline Methanol & Isopropanol & 65.71121 & -89.74272 & 0.304 \\
\hline \multicolumn{5}{|c|}{ water/ethanol/methanol } \\
\hline Water & Ethanol & 624.9174 & -29.169 & 0.2937 \\
\hline Water & Methanol & 594.6299 & -182.605 & 0.297 \\
\hline Ethanol & Methanol & 73.413 & -79.1718 & 0.3029 \\
\hline \multicolumn{5}{|c|}{ water/ethanol/cyclohexane } \\
\hline Water & Ethanol & 557.4826 & 29.08636 & 0.3475 \\
\hline Water & Cyclohexane & 4422.3 & 1688.273 & 0.21159 \\
\hline Ethanol & Cyclohexane & 440.6134 & 717.6762 & 0.46261 \\
\hline \multicolumn{5}{|c|}{ water/acetone/toluene } \\
\hline Water & Acetone & 653.885 & 377.577 & 0.5859 \\
\hline Water & Toluene & 2160.78 & 2839.37 & 0.2 \\
\hline Acetone & Toluene & -124.774 & 366.098 & 0.295 \\
\hline \multicolumn{5}{|c|}{ water/ethanol/methylacetate } \\
\hline Water & Ethanol & 624.9174 & -29.169 & 0.2937 \\
\hline Water & Methylacetate & 796.8165 & 334.6706 & 0.35 \\
\hline Ethanol & Methylacetate & 198.9705 & 134.162 & 0.3 \\
\hline
\end{tabular}


Table 16. Sieve tray distillation hardware details

\begin{tabular}{|l|l|}
\hline Parameter & value \\
\hline Column diameter & $0.8 \mathrm{~m}$ \\
\hline Tray spacing & $0.6 \mathrm{~m}$ \\
\hline Number of flow passes & 1 \\
\hline Liquid flow path length & $0.52 \mathrm{~m}$ \\
\hline Downcomer clearance & $0.038 \mathrm{~m}$ \\
\hline Deck thickness & $0.0025 \mathrm{~m}$ \\
\hline Hole diameter & $0.005 \mathrm{~m}$ \\
\hline Hole pitch & $0.012 \mathrm{~m}$ \\
\hline Active area & $76.0 \%$ \\
\hline Total hole area & $15.0 \%$ \\
\hline Downcomer area & $12.0 \%$ \\
\hline Weir type & Segmental \\
\hline Weir length & $0.7408 \mathrm{~m}$ \\
\hline Weir height & $0.05 \mathrm{~m}$ \\
\hline
\end{tabular}


Table 17. Specifications of operating conditions for design for water(1)/ethanol(2)/acetone(3) mixtures.

\begin{tabular}{|l|l|}
\hline Parameter & Value \\
\hline Total pressure & $101.3 \mathrm{kPa}$ \\
\hline Pressure drop per tray & 0 \\
\hline Reflux ratio & 3 \\
\hline Bottoms flow rate & $1.75 \mathrm{~mol} \mathrm{~s}^{-1}$ \\
\hline Mole fractions in feed & $x_{1}=0.015$ \\
(left of distillation boundary) & $x_{2}=0.25$ \\
& $x_{3}=0.735$ \\
\hline Mole fractions in feed & $x_{1}=0.2$ \\
(right of distillation boundary) & $x_{2}=0.05$ \\
& $x_{3}=0.75$ \\
\hline
\end{tabular}

Table 18. NRTL parameters for water(1)/ethanol(2)/acetone (3). These parameters are from the DECHEMA Dortmund data bank, as reported in Table 1 of Springer et al. ${ }^{23}$ These parameters are used along with $G_{i j}=\exp \left(-\alpha_{i j} \tau_{i j}\right)$ and $\tau_{i j}=B_{i j} / T$.

\begin{tabular}{ll|ccc}
\hline Component i & Component $\mathrm{j}$ & $B_{\mathrm{ij}} / \mathrm{K}$ & $B_{\mathrm{ji}} / \mathrm{K}$ & $\alpha_{\mathrm{ij}}$ \\
\hline Water & Ethanol & 624.9174 & -29.169 & 0.2937 \\
Water & Acetone & 602.6252 & 330.4768 & 0.5103 \\
Ethanol & Acetone & 188.8983 & 22.83319 & 0.3006
\end{tabular}


Table 19. NRTL parameters for 2-propanol(1)/water (2) mixture. These parameters are from the DECHEMA Dortmund data bank, and are used along with $G_{i j}=\exp \left(-\alpha_{i j} \tau_{i j}\right)$.

\begin{tabular}{ll|ccc}
\hline Component 1 & Component 2 & $\tau_{12}$ & $\tau_{21}$ & $\alpha_{12}$ \\
\hline $\begin{array}{l}\text { 2- } \\
\text { propanol(1) }\end{array}$ & water (2) & $\tau_{12}=\frac{70.6619}{T}$ & $\tau_{21}=\frac{729.2208}{T}$ & 0.288 \\
& & &
\end{tabular}

Table 20. NRTL parameters for ethanol(1)/water (2) mixture. These parameters are from the DECHEMA Dortmund data bank, and are used along with $G_{i j}=\exp \left(-\alpha_{i j} \tau_{i j}\right)$.

\begin{tabular}{ll|ccc}
\hline Component 1 & Component 2 & $\tau_{12}$ & $\tau_{21}$ & $\alpha_{12}$ \\
\hline ethanol(1) & water (2) & $\tau_{12}=-\frac{29.169}{T}$ & $\tau_{21}=\frac{624.9174}{T}$ & 0.2937
\end{tabular}

Table 21. NRTL parameters for acetone(1)/methanol (2) mixture. These parameters are from the Kurihara et al. ${ }^{48}$, and are used along with $G_{i j}=\exp \left(-\alpha_{i j} \tau_{i j}\right)$.

\begin{tabular}{ll|ccc}
\hline Component 1 & Component 2 & $\tau_{12}$ & $\tau_{21}$ & $\alpha_{12}$ \\
\hline ethanol(1) & water (2) & $\tau_{12}=\frac{770.15}{R T}$ & $\tau_{21}=\frac{1023.18}{R T}$ & 0.1099
\end{tabular}




\section{References}

(1) PTC MathCad 15.0, http://www.ptc.com/, PTC Corporate Headquarters, Needham, 3 November 2015.

(2) Krishna, R.; Goswami, A. N.; Nanoti, S. M.; Rawat, B. S.; Khanna, M. K.; Dobhal, J. Extraction of aromatics from 63-69 ${ }^{\circ} \mathrm{C}$ Naphtha fraction for food grade hexane production using sulpholane and NMP as solvents, Indian Journal of Technology 1987, 25, 602-606.

(3) Seader, J. D.; Henley, E. J.; Roper, D. K. Separation Process Principles; 3rd Edition, John Wiley: New York, 2011.

(4) Taylor, R.; Krishna, R. Multicomponent mass transfer; John Wiley: New York, 1993.

(5) Krishna, R.; Low, C. Y.; Newsham, D. M. T.; Olivera Fuentes, C. G.; Paybarah, A. Liquid Liquid Equilibrium in the System Glycerol Water Acetone at $25^{\circ} \mathrm{C}$, Fluid Phase Equilib. 1989, 45, 115120.

(6) Krishna, R. Serpentine Diffusion Trajectories and the Ouzo Effect in Partially Miscible Ternary Liquid Mixtures, Phys. Chem. Chem. Phys. 2015, 17, 27428-27436.

(7) Geddes, R. L. Local efficiencies of bubble-plate fractionators, Trans. Am. Inst. Chem. Engrs. 1946, 42, 79-105.

(8) Krishna, R.; Nanoti, S. M.; Goswam, A. N. Mass-Transfer Efficiency of Sieve Tray Extraction Columns, Ind. Eng. Chem. Res. 1989, 28, 642-644.

(9) Treybal, R. E. Mass-Transfer Operations; 3rd Edition, McGraw-Hill: New York, 1980.

(10) Robbins, L. A.; Cusack, R. W. Chaper 15, Liquid-Liquid Extraction Operations and Equipment. Perry's Chemical Engineers' Handbook; 7th Edition, Edited by R.H. Perry and D.W. Green, McGraw-Hill: New York, 1999.

(11) Krishna, R.; van Baten, J. M. The Darken relation for multicomponent diffusion in liquid mixtures of linear alkanes. An investigation using Molecular Dynamics (MD) simulations, Ind. Eng. Chem. Res. 2005, 44, 6939-6947.

(12) Reid, R.C.; Prausnitz, J. M.; Poling, B. E. The Properties of Gases and Liquids; 4th Edition, McGraw-Hill: New York, 1986.

(13) Kronig, R.; Brink, J. C. On the Theory of Extraction from Falling Droplets, Appl. Sci. Res. 1950, $A 2,142-154$.

(14) Sideman, S.; Shabtai, H. Direct-Contact Heat Transfer Between a Single Drop and an Immiscible Liquid Medium, Can. J. Chem. Eng. 1964, 42, 107-116.

(15) Sherwood, T. K.; Pigford, R. L.; Wilke, C. R. Mass Transfer; Mc-Graw Hill: New York, U.S.A., 1975.

(16) Cussler, E. L. Diffusion: Mass Transfer in Fluid Systems; 3rd Edition, Cambridge University Press: Cambridge, 2007.

(17) Krishna, R.; Low, C. Y.; Newsham, D. M. T.; Olivera-Fuentes, C. G.; Standart, G. L. Ternary mass transfer in liquid-liquid extraction, Chem. Eng. Sci. 1985, 40, 893-903.

(18) Taylor, R.; Krishna, R.; Kooijman, H. Real-World Modeling of Distillation, Chem. Eng. Prog. 2003, 99 (7), 28-39.

(19) Krishna, R.; Martinez, H. F.; Sreedhar, R.; Standart, G. L. Murphree point efficiencies in multicomponent systems, Trans. Inst. Chem. Eng. 1977, 55, 178-183.

(20) Krishna, R.; Standart, G. L. Mass and energy transfer in multicomponent systems, Chem. Eng. Commun. 1979, 3, 201-275. 
(21) Springer, P. A. M.; Baur, R.; Krishna, R. Influence of interphase mass transfer on the composition trajectories and crossing of boundaries in ternary azeotropic distillation, Sep. Purif. Technol. 2002, 29, 1-13.

(22) Springer, P. A. M.; Baur, R.; Krishna, R. Composition trajectories for heterogeneous azeotropic distillation in a bubble-cap tray column: Influence of mass transfer, Chem. Eng. Res. Des. 2003, 81, 413-426.

(23) Springer, P. A. M.; Buttinger, B.; Baur, R.; Krishna, R. Crossing of the distillation boundary in homogeneous azeotropic distillation: Influence of interphase mass transfer, Ind. Eng. Chem. Res. 2002, 41, 1621-1631.

(24) Springer, P. A. M.; Krishna, R. Crossing of boundaries in ternary azeotropic distillation: Influence of interphase mass transfer, Int. Commun. Heat Mass Transf. 2001, 28, 347-356.

(25) Springer, P. A. M.; van der Molen, S.; Baur, R.; Krishna, R. Experimental verification of the necessity to use the Maxwell-Stefan formulation in describing trajectories during azeotropic distillation, Chem. Eng. Res. Des. 2002, 80, 654-666.

(26) Springer, P. A. M.; van der Molen, S.; Krishna, R. The need for using rigorous rate-based models for simulations of ternary azeotropic distillation, Comput. Chem. Eng. 2002, 26, 1265-1279.

(27) Baur, R.; Taylor, R.; Krishna, R.; Copati, J. A. Influence of mass transfer in distillation of mixtures with a distillation boundary, Chem. Eng. Res. Des. 1999, 77, 561-565.

(28) Levy, S. G.; Van Dongen, D. B.; Doherty, M. F. Design and synthesis of homogeneous azeotropic distillation. 2. Minimum reflux calculations for nonideal and azeotropic columns, Ind. Eng. Chem. Fundamentals 1985, 24, 463-474.

(29) Li, Y. H.; Chen, H. F.; Liu, J. Q. Composition profile of an azeotropic continuous distillation with feed composition on a ridge or in a valley, Ind. Eng. Chem. Res. 1999, 38, 2482-2484.

(30) Kooijman, H. A.; Taylor, R. The ChemSep Book; 2nd Edition, www.chemsep.com: 2006.

(31) Krishna, R. Model for prediction of point efficiencies for multicomponent distillation, 1985, 63, 312-322.

(32) Fuller, E. N.; Schettler, P. D.; Giddings, J. C. A new method for prediction of binary gasphase diffusion coefficients, Ind. Eng. Chem. 1966, 58, 19-27.

(33) Springer, P. A. M. Mass Transfer Effects in Distillation, University of Amsterdam, Amsterdam, 2004.

(34) Fullarton, D.; Schlünder, E. U. Diffusion Distillation - A New Separation .Process for Azeotropic Mixtures - Part I: Selectivity and Transfer Efficiency, Chem. Eng. Process. 1986, 20, $255-$ 263.

(35) Singh, N.; Prasad, R. Experimental studies on the effect of inert gases on diffusion distillation of ethanol-water mixtures, J. Chem. Technol. Biotechnol. 2011, 86, 1495-1500.

(36) Meindersma, G. W. Extraction of Aromatics from Naphtha with Ionic Liquids, Ph.D. Dissertation, University of Twente, The Nethelands, Enschede, 2005.

(37) Al-Jimaz, A. S.; Fandary, M. S.; Al-Kandary, J. A.; Fahim, M. A. Liquid-liquid equilibria for n-alkanes $(\mathrm{C} 12, \mathrm{C} 14, \mathrm{C} 17)+$ propylbenzene +NMP mixtures at temperatures between 298 and $328 \mathrm{~K}$, Fluid Phase Equilib. 2005, 231, 163-170.

(38) Zuber, A.; Raimundo, R.; Mafra, M. R.; Filho, L. C.; Oliveira, J. V.; Corazza, M. L. Thermodynamic Modeling of Ternary Liquid-Liquid Systems with Forming Immiscibility Islands, Braz. Arch. Biol. Technol. 2013, 56, 1034-1042.

(39) Pertler, M. Die Mehrkomponenten-Diffuson in nicht vollständig mischbaren Flüssigkeiten, Technische Universität München, München, 1996.

(40) Bollen, A. M. Collected tales on mass transfer in liquids, Ph.D. Dissertation, Rijksuniversiteit Groningen, Groningen, 1999.

http://dissertations.ub.rug.nl/faculties/science/1999/a.m.bollen/

(41) Colombo, A.; Battilana, P.; Ragaini, V.; Bianchi, C. L. Liquid-Liquid Equilibria of the Ternary Systems Water + Acetic Acid + Ethyl Acetate and Water + Acetic Acid + Isophorone (3,5,5Trimethyl-2-cyclohexen-1-one), J. Chem. Eng. Data 1999, 44, 35-39. 
(42) Zhang, H.; Wang, T. Measurement and Correlation of Liquid-Liquid Equilibrium Data for Water + Acetic Acid + Methyl tert-Butyl Ether + NaCl, J. Chem. Eng. Data 2009, 54, 945-949.

(43) Aznar, M. Correlation of (Liquid + Liquid) Equilibrium of Systems Including Ionic Liquids, Braz. J. Chem. Eng. 2007, 24, 143-149.

(44) Santiago, R. S.; Santos, G. R.; Aznar, M. UNIQUAC correlation of liquid-liquid equilibrium in systems involving ionic liquids: The DFT-PCM approach, Fluid Phase Equilib. 2009, 278, 54-61.

(45) Wang, H.; Wang, Q.; Xiong, Z.; Chen, C. Liquid-liquid equilibria for ternary system water + toluene + benzaldehyde at (303.2-343.2) K, Fluid Phase Equilib. 2014, 383, 43-48.

(46) Ruiz, F.; Prats, D.; Gomis, V. Quaternary Liquid-Liquid Equilibrium: Water-EthanolChtoroformToluene at $25{ }^{\circ} \mathrm{C}$. Experimental Determination and Graphical and Analytical Correlation of Equillbrium Data, J. Chem. Eng. Data 1985, 30, 412-416.

(47) Washburn, E. R.; Beguin, A. E.; Beckford, O. C. The Ternary System: Ethyl Alcohol, Toluene and Water at 25, J. Am. Chem. Soc. 1939, 61, 1694-1695.

(48) Kurihara, K.; Hori, H.; Kojima, K. Vapor-Liquid Equilibrium Data for Acetone + Methanol + Benzene, Chloroform + Methanol + Benzene, and Constituent Binary Systems at 101.3 KPa, J. Chem. Eng. Data 1998, 43, 264-268. 


\section{Caption for Figures}

Figure 1. Schematic showing a variety of applications of liquid extraction processes in petroleum refining.

Figure 2. Schematic of agitated, and un-agitated column contactors used for liquid-liquid extraction processes.

Figure 3. Schematic of single-stage contacting in sieve-tray column.

Figure 4. (a, b) Transient equilibration trajectories for the system heptane(1)/toluene(2)/sulpholane(3) at $348.2 \mathrm{~K}$. For the extract phase, the initial mole fractions in the drop are $x_{10}=0.0, x_{20}=0.0$, and $x_{30}=$ 1.0; the final equilibrium composition is $x_{1, \mathrm{eq}}=0.062684992, x_{2, \mathrm{eq}}=0.406432625$, and $x_{3, \mathrm{eq}}=0.53088$. (c) Plot of the component Murphree efficiencies in the hydrocarbon-rich heptane(1)/toluene(2)/sulpholane(3) mixture, $E_{\mathrm{i}}$, as function of the Fourier number. (d) The composition trajectory followed during S-E equilibration. (c) Calculations of the ratios $-k_{12} / k_{11}$, and $-k_{21} / k_{22}$ as a function of the Fourier number. The NRTL parameters are provided in Table 1.

Figure 5. Interphase mass transfer resistances in liquid-liquid extraction. 
Figure 6. (a) Transient equilibration trajectories for the system NMP(1)/propylbenzene(2)/tetradecane(3) at $298 \mathrm{~K}$. The initial mole fractions in the drop are $x_{10}=1.0$, $x_{20}=0.0$, and $x_{30}=0.0$. The final equilibrium composition is $x_{1, \mathrm{eq}}=0.689463372, x_{2, \mathrm{eq}}=0.208896097$, and $x_{3, \mathrm{eq}}=0.101640532$. (b) Plot of the component Murphree efficiencies in the extract phase, $E_{\mathrm{i}}$, as function of the Fourier number. (c) Calculations of the ratios $-k_{12} / k_{11}$, and $-k_{21} / k_{22}$ as a function of the Fourier number. The NRTL parameters are provided in Table 3.

Figure 7. Transient equilibration trajectories for the system glycerol(1)/acetone(2)/water(3) mixtures at $298 \mathrm{~K}$. For the acetone-rich phase (left hand side), the initial mole fractions in the drop are $x_{10}=0.0, x_{20}$ $=0.77$, and $x_{30}=0.23$; the final equilibrium composition is $x_{1, \mathrm{eq}}=0.042, x_{2, \mathrm{eq}}=0.894$, and $x_{3, \mathrm{eq}}=0.064$. For the glycerol-rich phase (right hand side), the initial mole fractions in the drop are $x_{10}=0.85, x_{20}=$ 0.0 , and $x_{30}=0.15$; the final equilibrium composition is $x_{1, \mathrm{eq}}=0.552, x_{2, \mathrm{eq}}=0.164$, and $x_{3, \mathrm{eq}}=0.284$. The experimental data for the equilibration paths for glycerol(1)/acetone(2)/water(3) mixture measured in a stirred Lewis cell by Krishna et al. ${ }^{17}$ are also indicated. The two trajectories are calculated using $[D]=|\Lambda|^{1 / 2}[\Gamma]$ with $|\Lambda|^{1 / 2}=\left(D_{1, \text { self }}\right)^{x_{1}}\left(D_{2, \text { self }}\right)^{x_{2}}\left(D_{3, \text { self }}\right)^{x_{3}}$, taking $D_{1, \text { self }}=0.01, D_{2, \text { self }}=3.2, D_{3, \text { self }}=0.5$ with units $10^{-9} \mathrm{~m}^{2} \mathrm{~s}^{-1}$; this diffusivity information has been derived from our earlier work. ${ }^{6}$ In these calculations, the equilibration trajectories are determined using the exponential decay model $\left(x-x_{e q}\right)=[Q]\left(x_{0}-x_{e q}\right) ; \quad[Q] \equiv \exp [-A[D] t]$. The phase equilibrium is determined from the NRTL parameters in Table 4.

Figure 8. Transient equilibration trajectories for the system water(1)/acetone(2)/ethylacetate(3) at 293 $\mathrm{K}$. The initial mole fractions in the drop are $x_{10}=0.1, x_{20}=0.25$, and $x_{30}=0.0$. The final equilibrium 
compositions is $x_{1, \mathrm{eq}}=0.354107973, x_{2, \mathrm{eq}}=0.307050572$, and $x_{3, \mathrm{eq}}=0.338841456$. The UNIQUAC parameters for calculation of the phase equilibrium thermodynamics are provided in Table 6 .

Figure 9. Transient equilibration trajectories for the system water(1)/acetone(2)/ethylacetate(3) at 293 $\mathrm{K}$. The initial mole fractions in the drop are binary acetone(2)/ethylacetate(3) mixtures of varying compositions. The final equilibrium composition is $x_{1, \mathrm{eq}}=0.354107973, x_{2, \mathrm{eq}}=0.307050572$, and $x_{3, \mathrm{eq}}$ $=0.338841456$. The UNIQUAC parameters for calculation of the phase equilibrium thermodynamics are provided in Table 6.

Figure 10. Transient equilibration trajectories for the system water(1)/caprolactam(2)/toluene(3) at 298 K. The initial mole fractions in the drop are $x_{10}=0.0, x_{20}=0.6$, and $x_{30}=0.4$. The final equilibrium composition is $x_{1, \mathrm{eq}}=0.087810088, x_{2, \mathrm{eq}}=0.108474359$, and $x_{3, \mathrm{eq}}=0.803715553$. The UNIQUAC parameters for calculation of the phase equilibrium thermodynamics are provided in Table 7.

Figure 11. Transient equilibration trajectories for the system water(1)/caprolactam(2)/toluene(3) at 298 K. The initial mole fractions in the drop are binary caprolactam(2)/toluene(3) mixtures of varying compositions. The final equilibrium composition is $x_{1, \mathrm{eq}}=0.76316675, x_{2, \mathrm{eq}}=0.200866022$, and $x_{3, \mathrm{eq}}=$ 0.035967228. The UNIQUAC parameters for calculation of the phase equilibrium thermodynamics are provided in Table 7.

Figure 12. Transient equilibration trajectories for the system water(1)/acetic acid(2)/isophorone(3) at $298 \mathrm{~K}$. The initial mole fractions in the drop are $x_{10}=0.0, x_{20}=0.0$, and $x_{30}=1.0$. The final equilibrium 
composition is $x_{1, \mathrm{eq}}=0.544129988, x_{2, \mathrm{eq}}=0.223048947$, and $x_{3, \mathrm{eq}}=0.23282106$. The NRTL parameters for calculation of the phase equilibrium thermodynamics are provided in Table 8.

Figure 13. Transient equilibration trajectories for the system water(1)/acetic acid(2)/isophorone(3) at $298 \mathrm{~K}$. The initial mole fractions in the drop are binary acetic acid(2)/isophorone(3) mixtures of varying compositions. The final equilibrium composition is $x_{1, \mathrm{eq}}=0.544129988, x_{2, \mathrm{eq}}=0.223048947$, and $x_{3, \mathrm{eq}}=0.23282106$. The NRTL parameters for calculation of the phase equilibrium thermodynamics are provided in Table 8 .

Figure 14. Transient equilibration trajectories for the system water(1)/acetic acid(2)/MTBE(3) at 298.15 K. The initial mole fractions in the drop are $x_{10}=0.0, x_{20}=0.0$, and $x_{30}=1$. The final equilibrium composition is $x_{1, \mathrm{eq}}=0.424393, x_{2, \mathrm{eq}}=0.243211571$, and $x_{3, \mathrm{eq}}=0.332395429$. The NRTL parameters for calculation of the phase equilibrium thermodynamics are provided in Table 9.

Figure 15. Transient equilibration trajectories for the system water(1)/acetic acid(2)/MTBE(3) at 298.15 K. The initial mole fractions in the drop are binary acetic acid(2)/MTBE(3) mixtures of varying compositions. The final equilibrium composition is $x_{1, \mathrm{eq}}=0.424393, x_{2, \mathrm{eq}}=0.243211571$, and $x_{3, \mathrm{eq}}=$ 0.332395429. The NRTL parameters for calculation of the phase equilibrium thermodynamics are provided in Table 9.

Figure 16. Transient equilibration trajectory for the system $[\mathrm{omim}][\mathrm{Cl}](1) / \mathrm{ethanol}(2) / \mathrm{TAEE}(3)$ at 298.15. Here we denote the ionic liquid 1-octyl-3-methylimidazolium chloride in the abbreviated form 
[omim $][\mathrm{Cl}]$. TAEE is the abbreviated name for tert-amyl ethyl ether. The initial mole fractions in the drop are $x_{10}=1.0, x_{20}=0.0$, and $x_{30}=0.0$. The final equilibrium composition is $x_{1, \mathrm{eq}}=0.150072736$, $x_{2, \text { eq }}=0.47026855$, and $x_{3, \text { eq }}=0.379658714$. The NRTL parameters are provided in Table 10.

Figure 17. Transient equilibration trajectory for the system $[\mathrm{omim}][\mathrm{Cl}](1) / \mathrm{ethanol}(2) / \mathrm{TAEE}(3)$ at 298.15. Here we denote the ionic liquid 1-octyl-3-methylimidazolium chloride in the abbreviated form [omim $][\mathrm{Cl}]$. TAEE is the abbreviated name for tert-amyl ethyl ether. The initial mole fractions in the drop are $x_{10}=1.0, x_{20}=0.0$, and $x_{30}=0.0$. The final equilibrium composition is $x_{1, \mathrm{eq}}=0.397919131$, $x_{2, \mathrm{eq}}=0.381690314$, and $x_{3, \mathrm{eq}}=0.22039056$. The NRTL parameters are provided in Table 10.

Figure 18. Transient equilibration trajectories for the system $[\mathrm{omim}][\mathrm{Cl}](1) / \mathrm{ethanol}(2) / \mathrm{TAEE}(3)$ at 298.15. Here we denote the ionic liquid 1-octyl-3-methylimidazolium chloride in the abbreviated form [omim] $[\mathrm{Cl}]$. TAEE is the abbreviated name for tert-amyl ethyl ether. The initial mole fractions in the drop are all devoid of TAEE, and contain different proportions of [omim $][\mathrm{Cl}]$ and ethanol(2). The final equilibrium composition for all starting compositions is $x_{1, \mathrm{eq}}=0.397919131, x_{2, \mathrm{eq}}=0.381690314$, and $x_{3, \mathrm{eq}}=0.22039056$. The NRTL parameters are provided in Table 10.

Figure 19. Transient equilibration trajectory for the system $[\mathrm{bmim}][\mathrm{TfO}](1) / \mathrm{ethanol}(2) / \mathrm{TAEE}(3)$ at 298.15 K. Here [bmim] $[$ TfO] $=$ 1-butyl-3-methylimidazolium trifluoromethanesulfonate. TAEE is the abbreviated name for tert-amyl ethyl ether. The initial mole fractions in the drop are $x_{10}=0.6, x_{20}=0.2$, and $x_{30}=0.0$. The final equilibrium composition is $x_{1, \mathrm{eq}}=0.316915772, x_{2, \mathrm{eq}}=0.361266258$, and $x_{3, \mathrm{eq}}=$ 0.32181797. The UNIQUAC parameters are provided in Table 11. 
Figure 20. Transient equilibration trajectories for the system [bmim][TfO](1)/ethanol(2)/TAEE(3) at $298.15 \mathrm{~K}$. Here $[\mathrm{bmim}][\mathrm{TfO}]=$ 1-butyl-3-methylimidazolium trifluoromethanesulfonate. TAEE is the abbreviated name for tert-amyl ethyl ether. The initial mole fractions in the drop contain varying compositions of the binary mixtures [bmim][TfO](1)/ethanol(2). The final equilibrium composition for all starting compositions is $x_{1, \mathrm{eq}}=0.397919131, x_{2, \mathrm{eq}}=0.381690314$, and $x_{3, \mathrm{eq}}=0.22039056$. The UNIQUAC parameters are provided in Table 11.

Figure 21. Transient equilibration trajectory for the system water(1)/ethanol(2)/cyclohexane(3) at 298 $\mathrm{K}$. The initial mole fractions in the drop are $x_{10}=0.3, x_{20}=0.7$, and $x_{30}=0.0$. The final equilibrium composition is $x_{1, \mathrm{eq}}=0.325889064, x_{2, \mathrm{eq}}=0.563182958$, and $x_{3, \mathrm{eq}}=0.11093$. The NRTL parameters for calculation of the phase equilibrium thermodynamics are provided in Table 12.

Figure 22. Transient equilibration trajectory for the system water(1)/ethanol(2)/cyclohexane(3) at 298 K. The initial mole fractions in the drop are binary water(1)/ethanol(2) mixtures of varying compositions. The final equilibrium composition is $x_{1, \mathrm{eq}}=0.325889064, x_{2, \mathrm{eq}}=0.563182958$, and $x_{3, \mathrm{eq}}=$ 0.11093. The NRTL parameters for calculation of the phase equilibrium thermodynamics are provided in Table 12.

Figure 23. Transient equilibration trajectory for the system toluene(1)/ethanol(2)/water(3) at $298 \mathrm{~K}$. The initial mole fractions in the drop are $x_{10}=0.25, x_{20}=0.75$, and $x_{30}=0.0$. The final equilibrium 
composition is $x_{1, \mathrm{eq}}=0.2952817, x_{2, \mathrm{eq}}=0.454727223$, and $x_{3, \mathrm{eq}}=0.24999108$. The NRTL parameters for toluene(1)/ethanol(2)/water(3) are provided in Table 13.

Figure 24. Transient equilibration trajectory for the system toluene(1)/ethanol(2)/water(3) at $298 \mathrm{~K}$. The initial mole fractions in the drop are binary toluene(1)/ethanol(2) mixtures of varying compositions. The final equilibrium composition is $x_{1, \mathrm{eq}}=0.2952817, x_{2, \mathrm{eq}}=0.454727223$, and $x_{3, \mathrm{eq}}=0.24999108$. The NRTL parameters for toluene(1)/ethanol(2)/water(3) are provided in Table 13.

Figure 25. Transient equilibration trajectory for the system water(1)/acetone(2)/toluene(3) at $298 \mathrm{~K}$. The initial mole fractions in the drop are $x_{10}=0.3, x_{20}=0.7$, and $x_{30}=0.0$. The final equilibrium composition is $x_{1, \mathrm{eq}}=0.057357706, x_{2, \mathrm{eq}}=0.600380623$, and $x_{3, \mathrm{eq}}=0.342261671$. The NRTL parameters for calculation of the phase equilibrium thermodynamics are provided in Table 14.

Figure 26. Transient equilibration trajectory for the system water(1)/acetone(2)/toluene(3) at $298 \mathrm{~K}$. The initial mole fractions in the drop are binary water(1)/acetone(2) mixtures of varying compositions. The final equilibrium composition is $x_{1, \mathrm{eq}}=0.057357706, x_{2, \mathrm{eq}}=0.600380623$, and $x_{3, \mathrm{eq}}=0.342261671$. The NRTL parameters for calculation of the phase equilibrium thermodynamics are provided in Table 14.

Figure 27. Transfer resistances in vapor/liquid contacting on a distillation tray. 
Figure 28. (a) Residue curve map for the Methanol - Isopropanol - Water system, showing a straightline distillation boundary and feed locations F1 and F2 on either side of the distillation boundary. (b) Residue curve map for the Acetone - Chloroform - Methanol system, showing feed locations F1 and F2 on the concave and convex sides of the highlighted distillation boundary respectively.

Figure 29. (a) Schematic of a laboratory-scale distillation column used in the experiments of Springer et al. $^{21,23}$ which includes a total condenser (1), a partial reboiler (12), 10 bubble-cap trays (2-11), and 13 draw-off faucets, 9 for vapor samples and 4 for liquid samples.

Figure 30. Residue curve maps for distillation of water(1)/ethanol(2)/acetone(3) mixtures. The blue circles represent the experimental data for T2-26 of Springer et al. ${ }^{21,23,25,26}$ on composition trajectories in a bubble-cap tray column operating at total reflux implying $x_{i}=y_{i}$.

Figure 31. (a) Ethanol driving force $\Delta y_{2 E}=\left(y_{2 E}-y_{2, e q}\right)$ on each stage for the system water(1)/ethanol(2)/acetone(3). (b) Murphree component efficiencies for the system water(1)/ethanol(2)/acetone(3). 
Figure 32. Residue curve maps for distillation of water(1)/ethanol(2)/methanol(3) mixtures. The blue circles represent the experimental data for T4-13 of Springer et al. ${ }^{21,23,25,26}$ on composition trajectories in a bubble-cap tray column operating at total reflux implying $x_{i}=y_{i}$.

Figure 33. Residue curve maps for distillation of water(1)/ethanol(2)/methylacetate(3) mixtures. The blue circles represent the experimental data for T3-23 of Springer et al. ${ }^{21,23,25,26}$ on composition trajectories in a bubble-cap tray column operating at total reflux implying $x_{i}=y_{i}$.

Figure 34. Experimental data (blue circles) of Springer et al. $^{25}$ for Run Q6 with quaternary water(1)/ethanol(2)/methanol(3)/acetone(4) mixtures. Also shown as insets are the Murphree component efficiencies and component driving forces.

Figure 35. (a) Transient equilibration trajectories for the system water(1)/ethanol(2)/acetone(3) at 335.5 K. The initial mole fractions in the rigid spherical vapor bubble are $y_{10}=0.067, y_{20}=0.44$, and $y_{30}=$ 0.493; the final equilibrium compositions are $y_{1, \mathrm{eq}}=0.04335, y_{2, \mathrm{eq}}=0.25907$, and $y_{3, \mathrm{eq}}=0.69758$. (b) Plot of the component Murphree efficiencies, $E_{\mathrm{i}}$, as function of the Fourier number. (c) NEQ and EQ trajectory vectors for various entering tray compositions. (d) The orange shaded region indicate vapor compositions that will have trajectories that cross the distillation boundary. 
Figure 36. Design result for feed left of distillation boundary. (a) Comparison of column composition trajectories calculated using the NEQ with EQ stage model. (b) Required number of stages for a bottom product of $96 \mathrm{~mol} \%$ ethanol. (c) Murphree component efficiencies.

Figure 37. Design result for feed right of distillation boundary. (a) Comparison of column composition trajectories calculated using the NEQ with EQ stage model. (b) Required number of stages for a bottom product of $100 \mathrm{~mol} \%$ water. (c) Murphree component efficiencies.

Figure 38. (a) Transient equilibration trajectories for the system water(1)/ethanol(2)/methanol(3) at 348 K. The initial mole fractions in the rigid spherical vapor bubble are $y_{10}=0.082, y_{20}=0.68$, and $y_{30}=$ 0.238 ; the final equilibrium compositions are $y_{1, \mathrm{eq}}=0.06767, y_{2, \mathrm{eq}}=0.59691$, and $y_{3, \mathrm{eq}}=0.33542$. (b) Plot of the component Murphree efficiencies, $E_{\mathrm{i}}$, as function of the Fourier number. (c) NEQ and EQ trajectory vectors for various entering tray compositions. (d) The orange shaded region indicate vapor compositions that will have trajectories that cross the distillation boundary.

Figure 39. (a) Transient equilibration trajectories for the system water(1)/ethanol(2)/methylacetate(3) at $337 \mathrm{~K}$. The initial mole fractions in the rigid spherical vapor bubble are $y_{10}=0.095, y_{20}=0.6345$, and $y_{30}=0.2705$; the final equilibrium compositions are $y_{1, \mathrm{eq}}=0.06324, y_{2, \mathrm{eq}}=0.36863$, and $y_{3, \mathrm{eq}}=0.56813$. (b) Plot of the component Murphree efficiencies, $E_{\mathrm{i}}$, as function of the Fourier number. (c) NEQ and EQ trajectory vectors for various entering tray compositions. (d) The orange shaded region indicate vapor compositions that will have trajectories that cross the distillation boundary. 
Figure 40. Residue curve maps for distillation of water(1)/acetone(2)/toluene(3) mixtures. The blue circles represent the experimental data for WAT-1 of Springer et al. ${ }^{22}$ (blue circles) showing the column composition trajectories in the bubble-cap tray column operating at total reflux implying $x_{i}=y_{i}$.

Figure 41. Residue curve maps for distillation of water(1)/acetone(2)/toluene(3) mixtures. The blue circles represent the experimental data for WAT-2 of Springer et al. ${ }^{22}$ (blue circles) showing the column composition trajectories in the bubble-cap tray column operating at total reflux implying $x_{i}=y_{i}$.

Figure 42. Residue curve maps for distillation of water(1)/ethanol(2)/cyclohexane(3) mixtures. The blue circles represent the experimental data for WEC-12 of Springer et al. ${ }^{22}$ (blue circles) showing the column composition trajectories in the bubble-cap tray operating at total reflux implying $x_{i}=y_{i}$.

Figure 43. Residue curve maps for distillation of water(1)/ethanol(2)/cyclohexane(3) mixtures. The blue circles represent the experimental data for WEC-8 of Springer et al. ${ }^{22}$ (blue circles) showing the column composition trajectories in the bubble-cap tray column operating at total reflux implying $x_{i}=y_{i}$.

Figure 44. Transfer resistances in heterogeneous azeotropic distillation. This scheme is adapted from Springer et al. ${ }^{22}$

Figure 45. Diffusional distillation of alcohol(1)-water(2) binary mixture by introduction of pure inert gas (3) at the inlet to the tray. 
Figure 46. Transient equilibration in the vapor phase determined using the Geddes model. (a) The mole fractions of 2-propanol (1) and water (2) plotted as a function of time, $t$. The total pressure is $101.3 \mathrm{kPa}$, and the temperature is $313.15 \mathrm{~K}$. The well-mixed liquid composition on the tray is $x_{1}=0.637, x_{2}=$ 0.363. The vapor composition entering the tray is $y_{1 \mathrm{E}}=0.0, y_{2 \mathrm{E}}=0.0, y_{3 \mathrm{E}}=1.0$. The composition of the vapor in equilibrium with the liquid is $y_{1, \mathrm{eq}}=0.09836, y_{2, \mathrm{eq}}=0.05963, y_{3 \mathrm{E}}=0.84201$. The phase equilibrium is calculated using the NRTL parameters provided in Table 19. (b) Ratio of the mole fraction of water (2) to that of 2-propanol (1) in the vapor phase as a function of time, $t$. (c) Comparisons of the ratios of the mole fraction of water (2) to that of 2-propanol (1) in the vapor phase as a function of time, $t$ using nitrogen, argon, and helium as inert gas. (d) Comparison of diffusion equilibration trajectories in composition space.

Figure 47. Transient equilibration in the vapor phase determined using the Geddes model. (a) The mole fractions of ethanol (1) and water (2) plotted as a function of time, $t$.The total pressure is $101.3 \mathrm{kPa}$, and the temperature is $313.15 \mathrm{~K}$. The well-mixed liquid composition on the tray is $x_{1}=0.87, x_{2}=0.13$. The vapor composition entering the tray is $y_{1 \mathrm{E}}=0.0, y_{2 \mathrm{E}}=0.0, y_{3 \mathrm{E}}=1.0$. The composition of the vapor in equilibrium with the liquid is $y_{1, \mathrm{eq}}=0.09859, y_{2, \mathrm{eq}}=0.02278, y_{3 \mathrm{E}}=0.82455$. The phase equilibrium is calculated using the NRTL parameters provided in Table 20. (b) Ratio of the mole fraction of water (2) to that of ethanol (1) in the vapor phase as a function of time, $t$. (c) Comparisons of the ratios of the mole fraction of water (2) to that of ethanol (1) in the vapor phase as a function of time, $t$ using nitrogen, argon, and helium as inert gas. (d) Comparison of diffusion equilibration trajectories in composition space.

Figure 48. Transient equilibration in the vapor phase determined using the Geddes model. (a) The mole fractions of acetone (1) and methanol (2) plotted as a function of time, $t$. The total pressure is $101.3 \mathrm{kPa}$, 
and the temperature is $313.15 \mathrm{~K}$. The well-mixed liquid composition on the tray is $x_{1}=0.85259, x_{2}=$ 0.14741. The vapor composition entering the tray is $y_{1 \mathrm{E}}=0.0, y_{2 \mathrm{E}}=0.0, y_{3 \mathrm{E}}=1.0$. The composition of the vapor in equilibrium with the liquid is $y_{1, \mathrm{eq}}=0.4798, y_{2, \mathrm{eq}}=0.08296, y_{3 \mathrm{E}}=0.43724$. The phase equilibrium is calculated using the NRTL parameters provided in Table 21. (b) Ratio of the mole fraction of methanol (2) to that of acetone (1) in the vapor phase as a function of time, $t$. (c) Comparisons of the ratios of the mole fraction of methanol (2) to that of acetone (1) in the vapor phase as a function of time, $t$ using nitrogen, argon, and helium as inert gas. (d) Comparison of diffusion equilibration trajectories in composition space. 


\section{LLE applications in Petroleum Refining ${ }^{\text {Fig. }}$ s1}

Liquid Extraction in Petroleum Refining

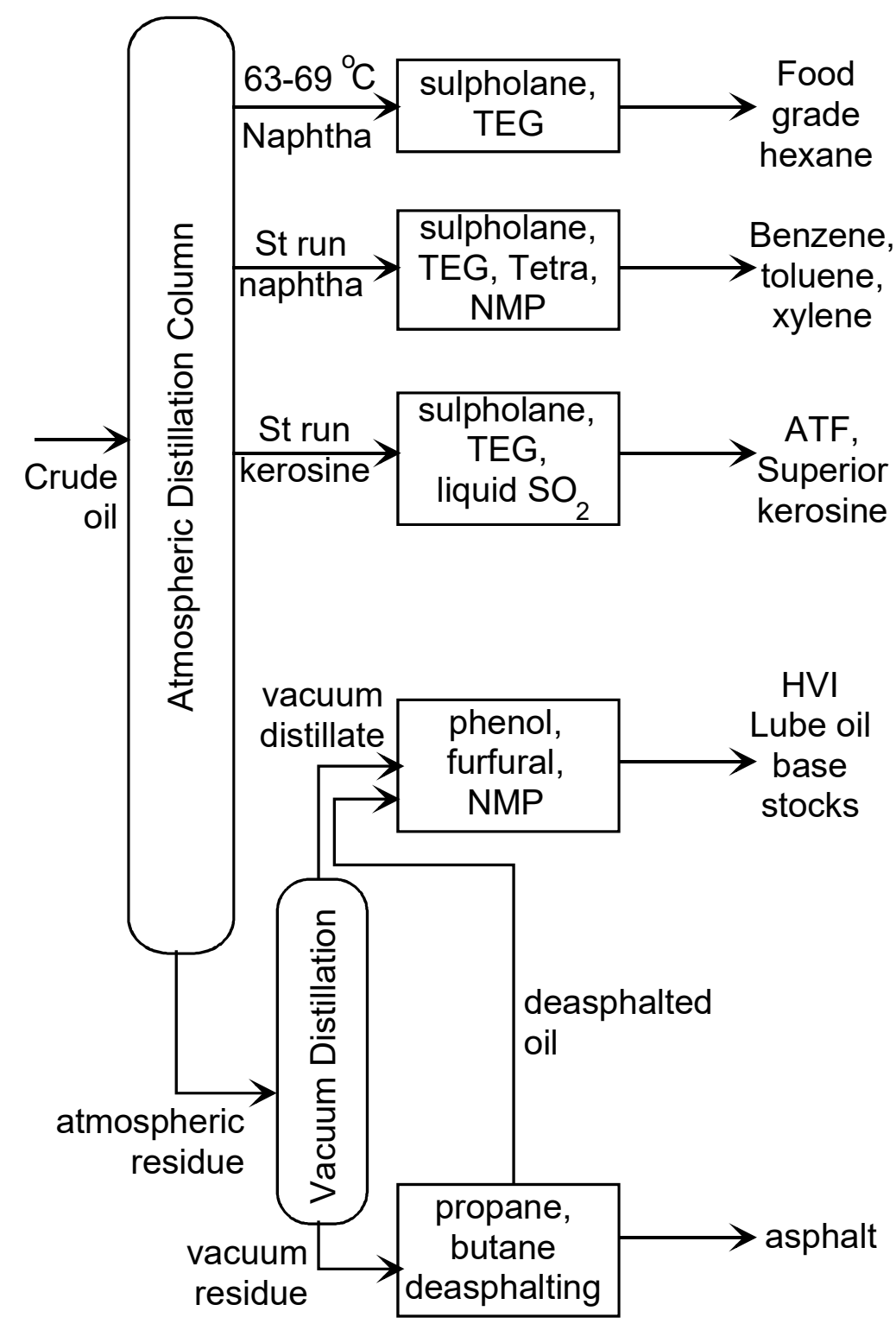




\title{
Agitated and Un-Agitated Contactors Fig. s2
}

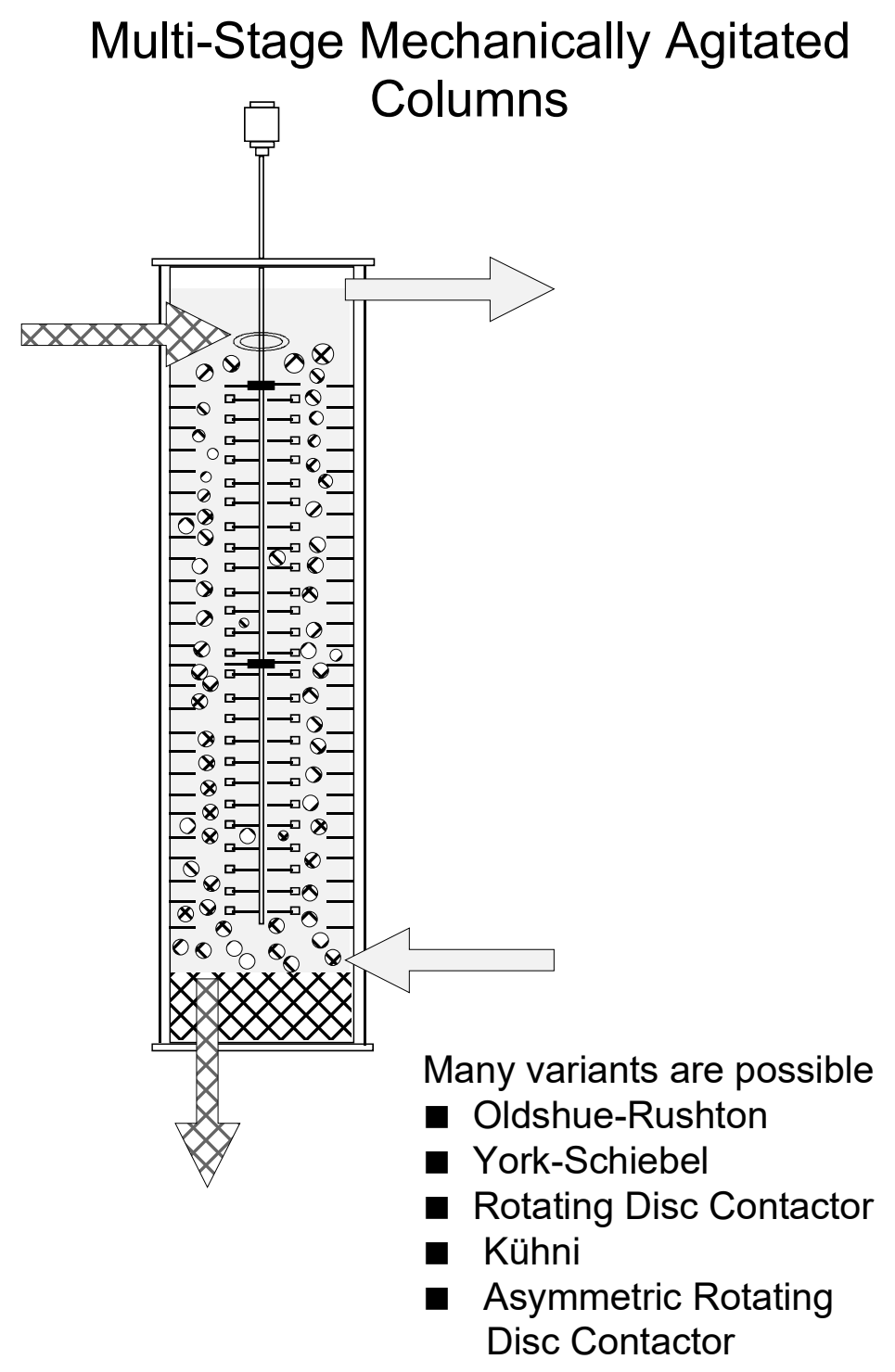

\author{
Un- Agitated Columns
}
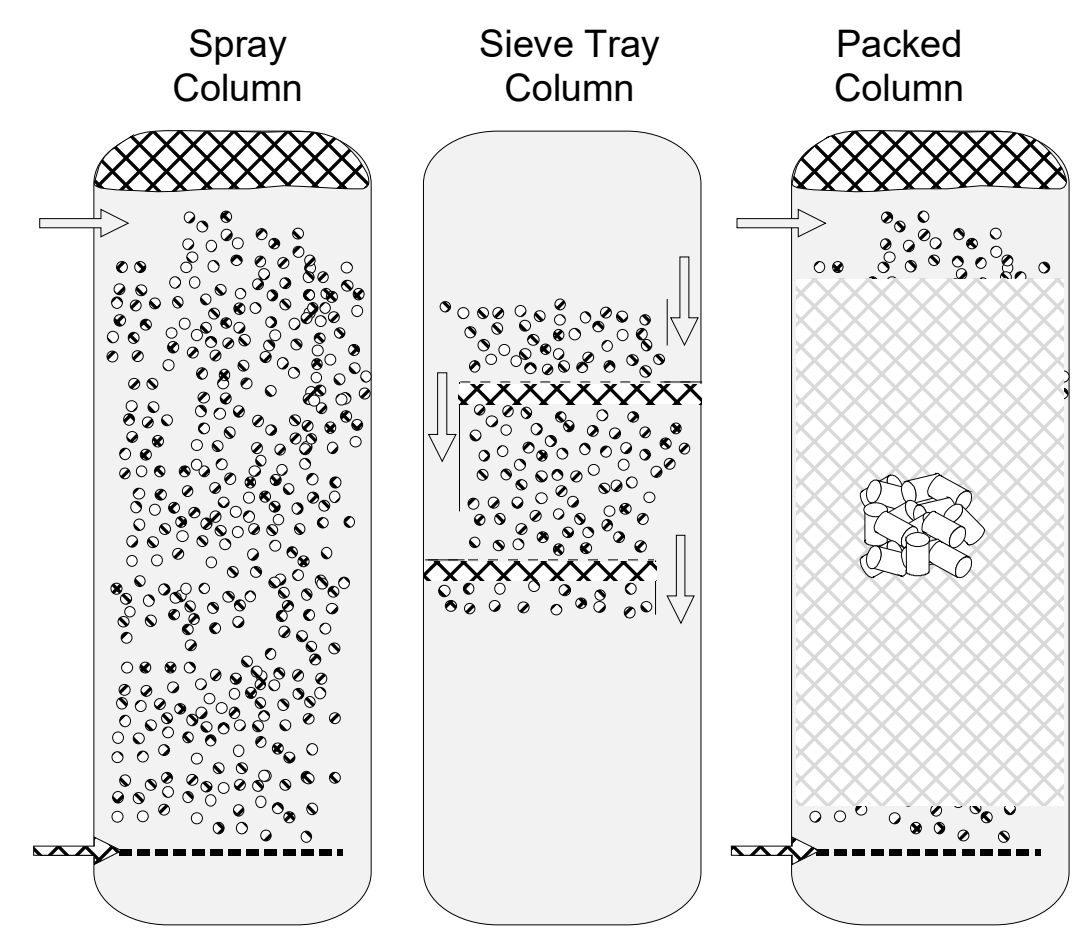

- Oldshue-Rushton

- York-Schiebel

- Rotating Disc Contactor

- Asymmetric Rotating

- large amount of backmixing

- some backof both phases mixing of drops

- channelling and maldistrbution in large diameter columns 


\section{Single stage mass transfer}

Fig. S3

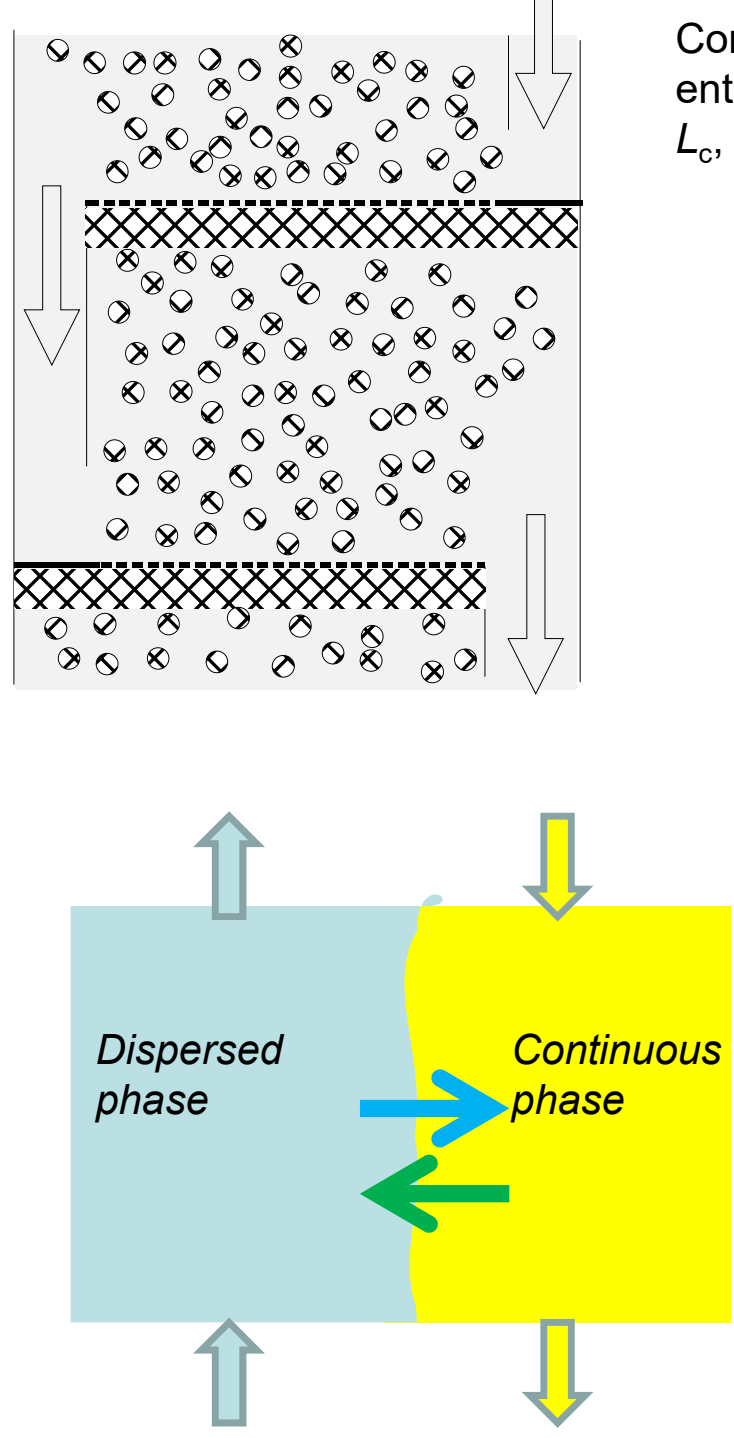

Interphase mass transfer
Continuous phase liquid entering

$L_{c}, x_{\mathrm{CE}}$

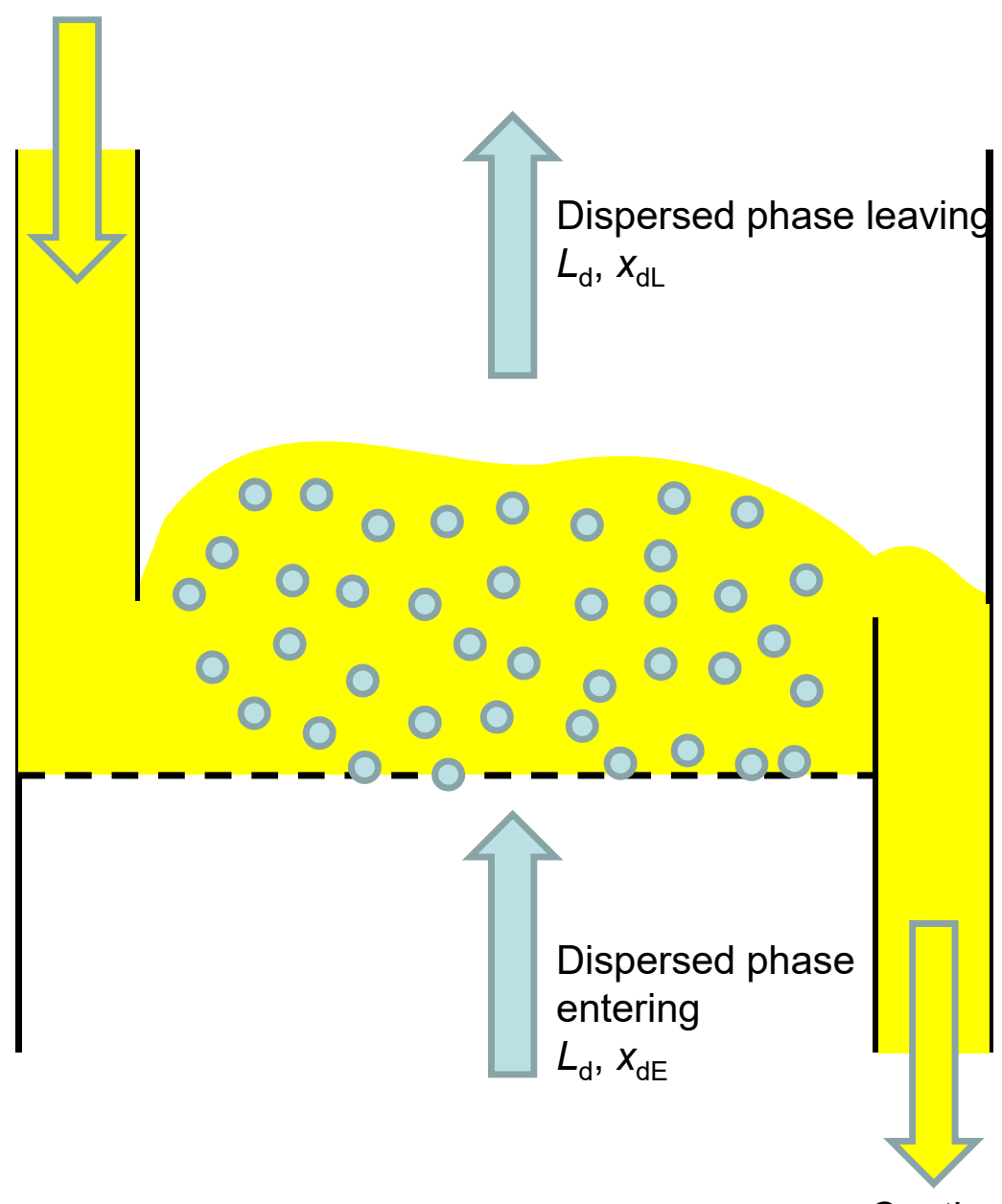

Continuous phase liquid leaving

$L_{c}, x_{\mathrm{cL}}$ 


\section{Heptane/toluene/sulpholane equilibratiofig. $\$ 4$}

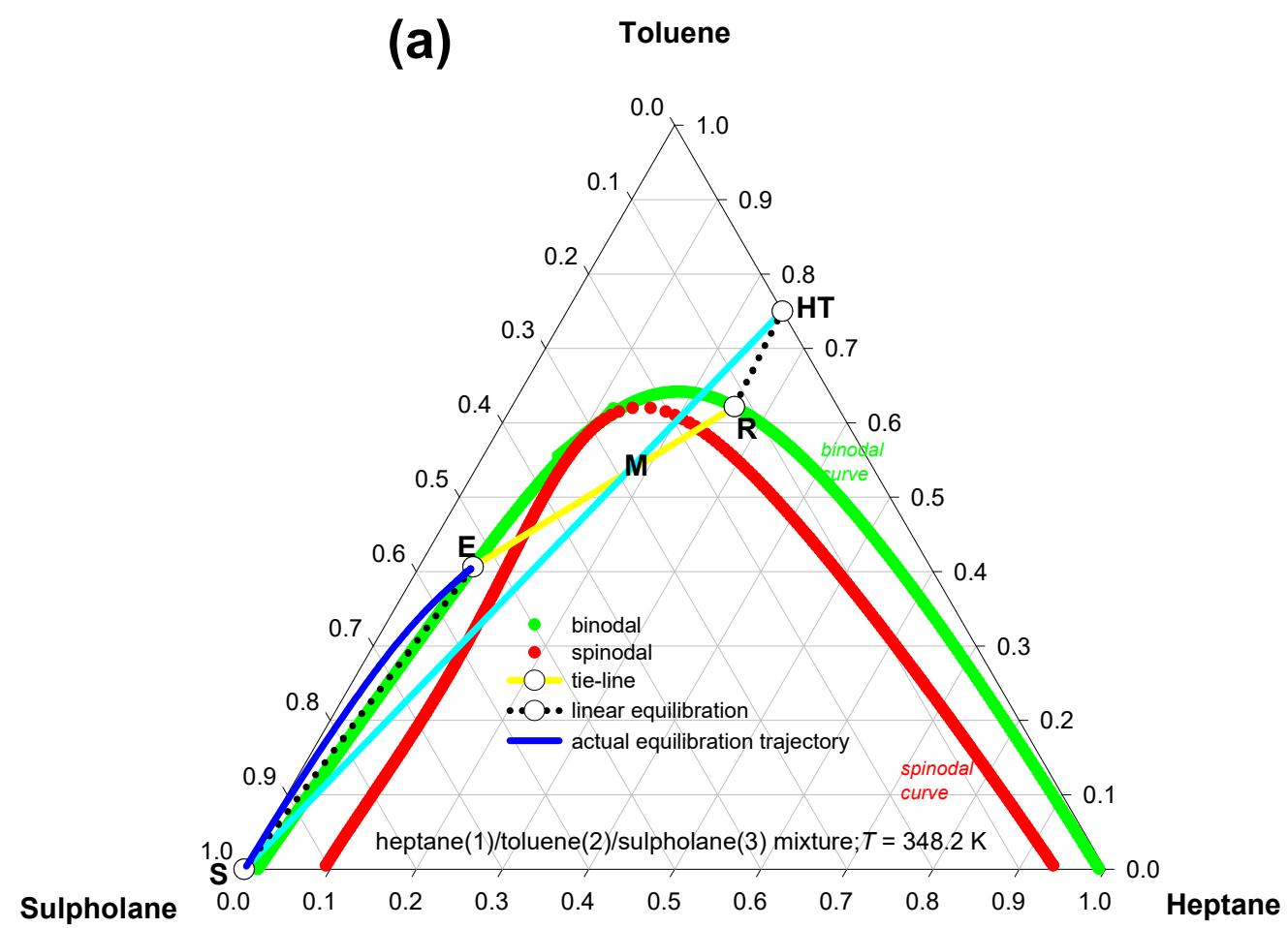

(b)

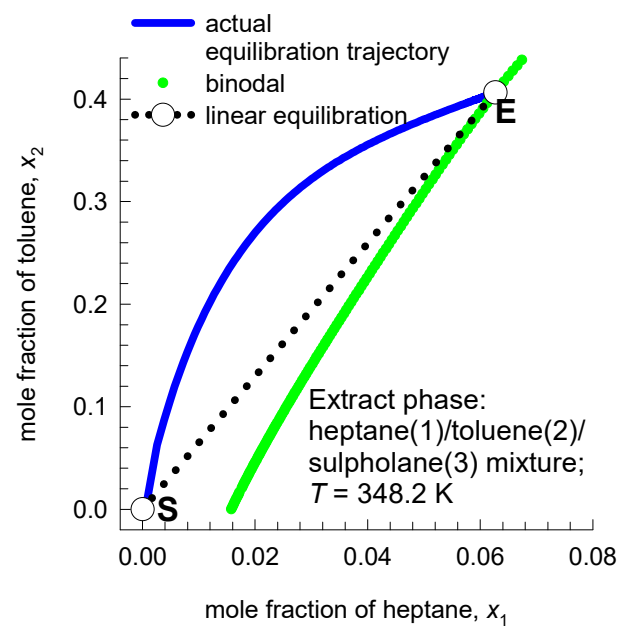

(c)

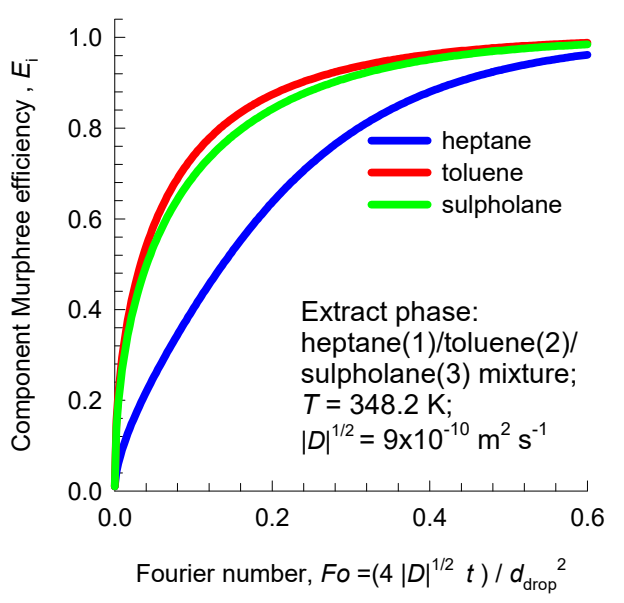

(d)

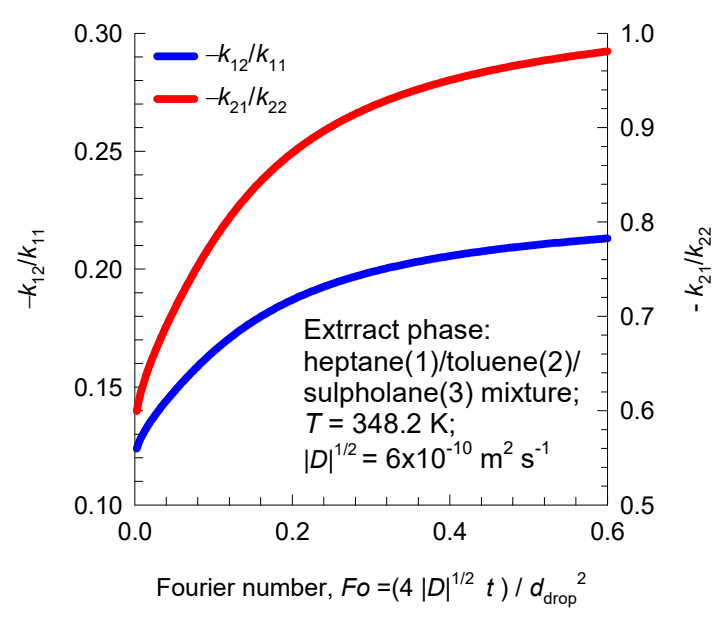




\section{Mass transfer resistances: Liquid-Liquid Extraction}

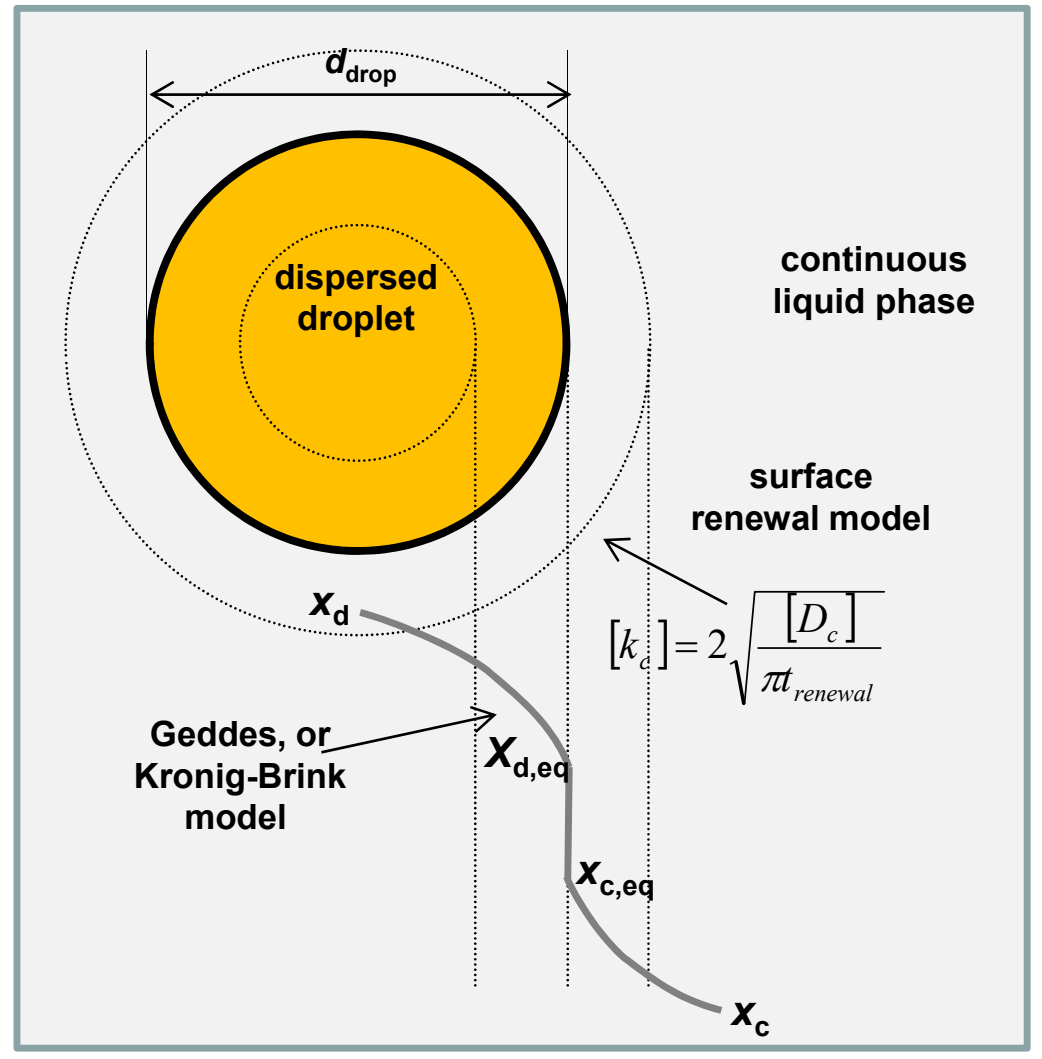




\section{NMP/propylbenzene/tetradecane equilibraffifor}

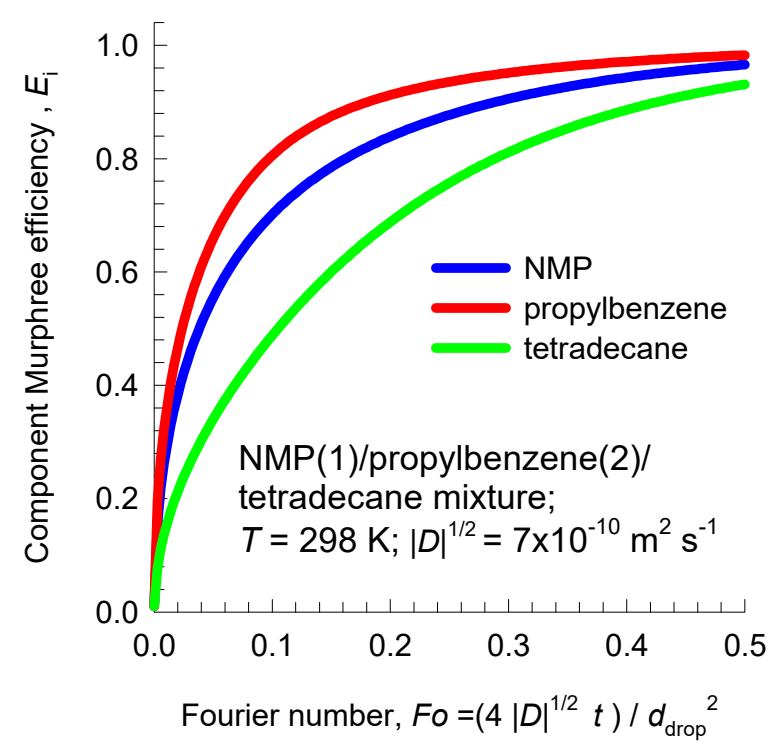

Propylbenzene

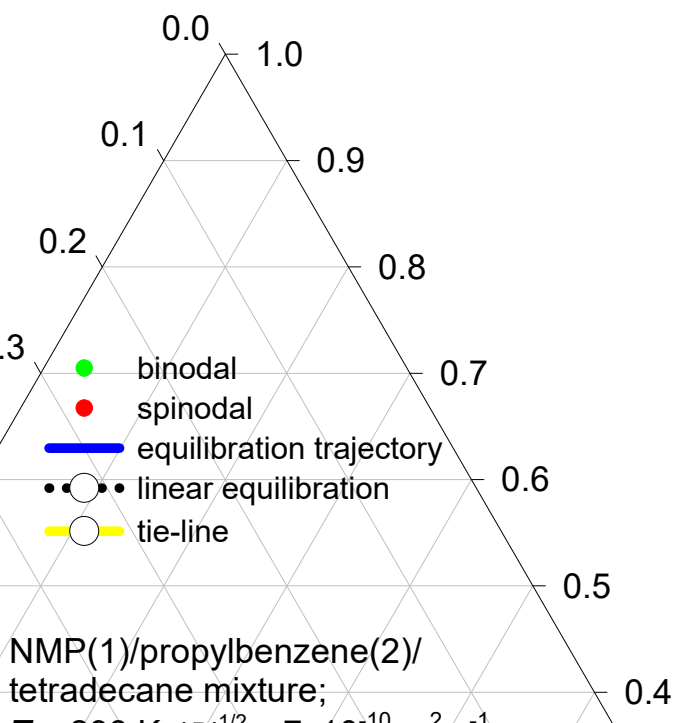

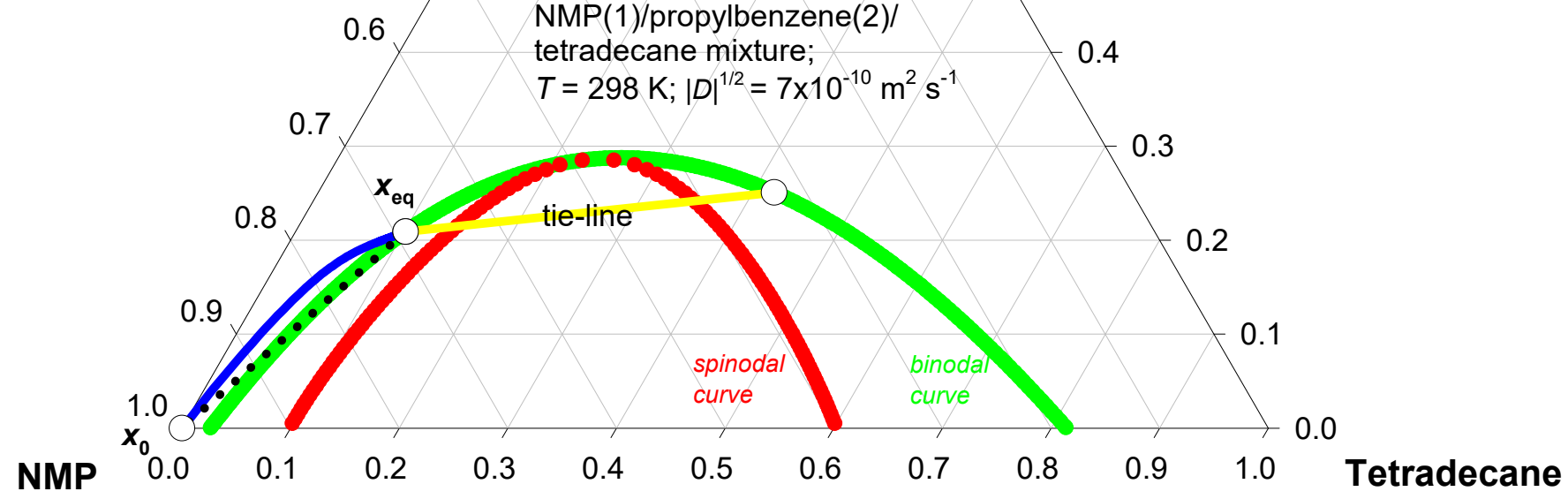




\section{Glycerol/Acetone/Water Equilibration Fig. s7}

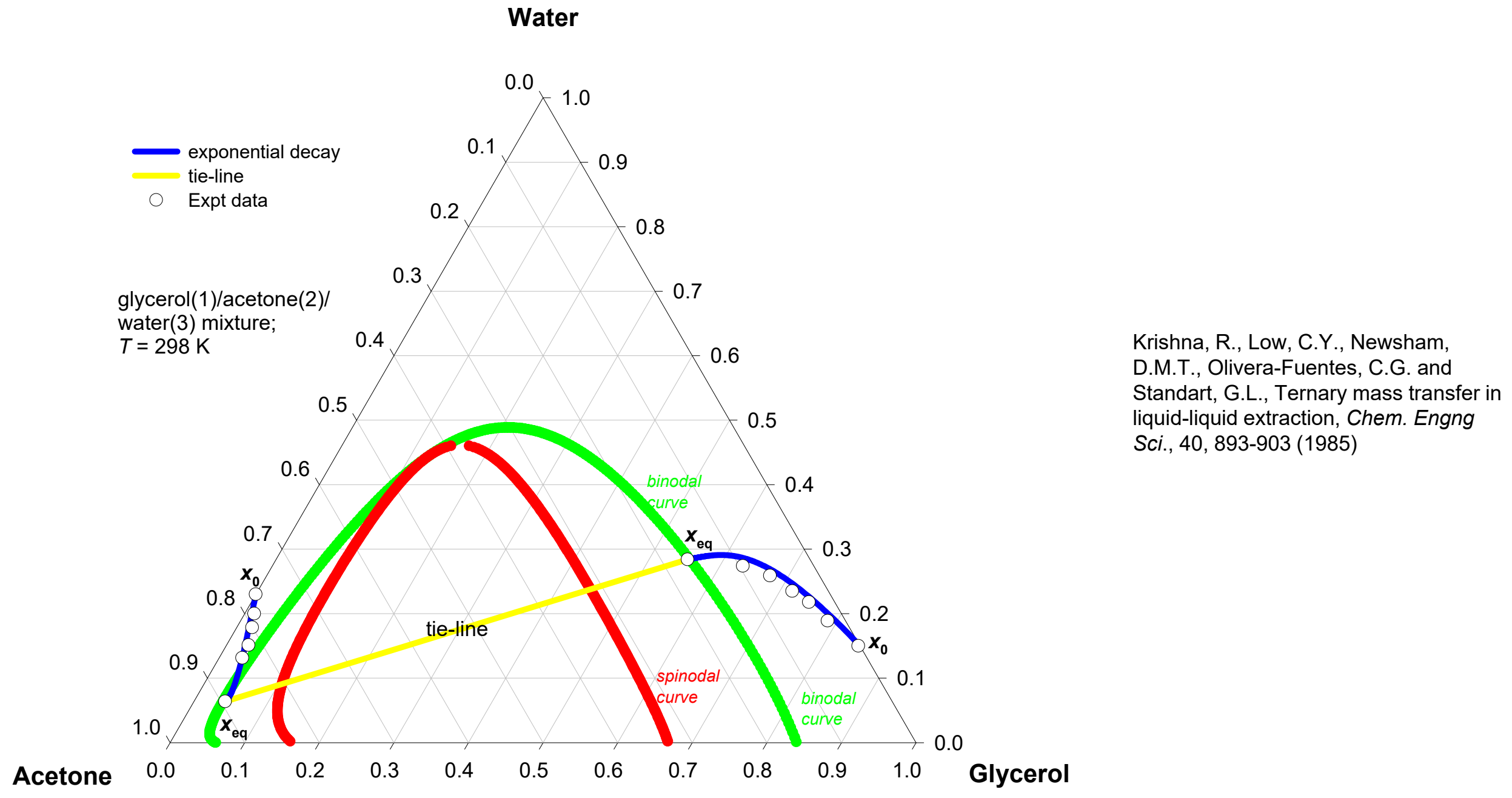




\section{Water/Acetone/Ethylacetate equilibration}

Fig. S8
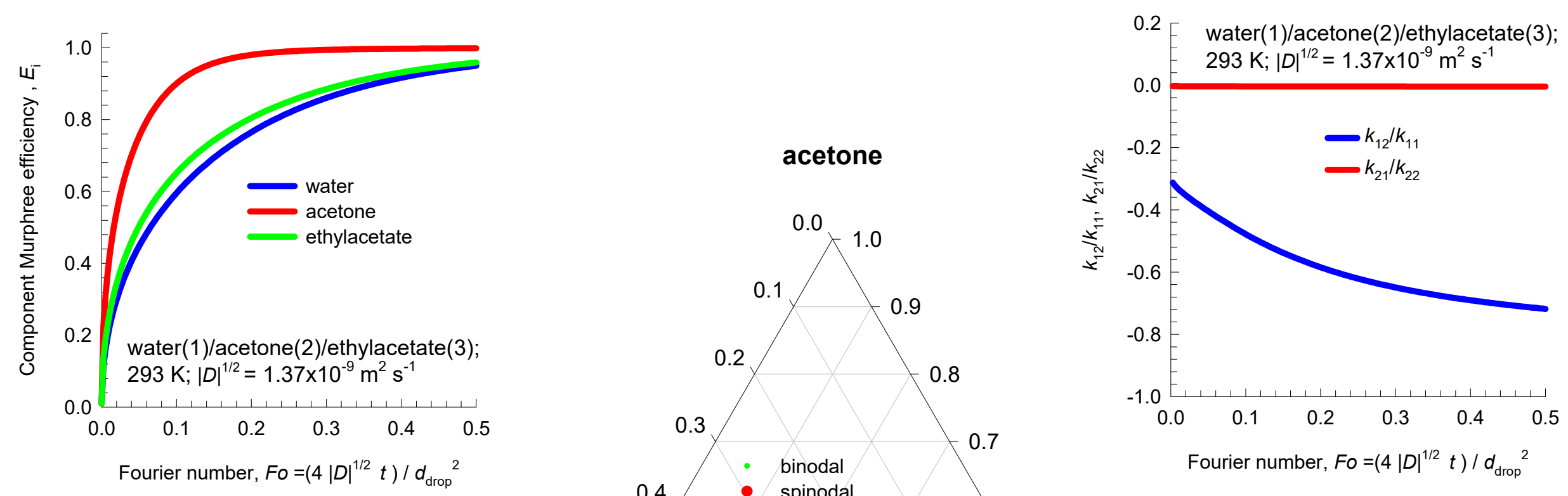

ethylacetate

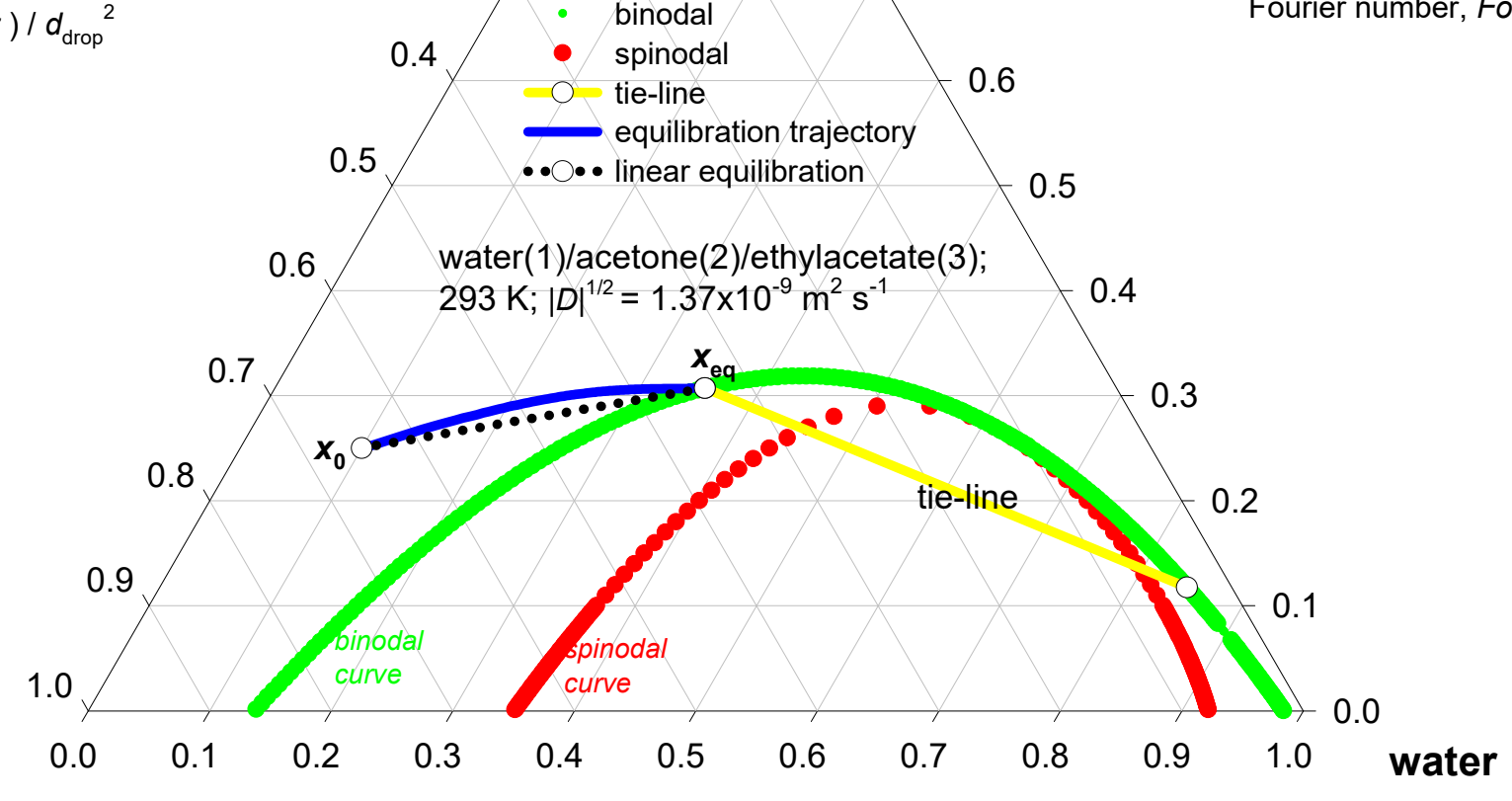


Fig. 59

\section{Water/Acetone/Ethylacetate equilibration}

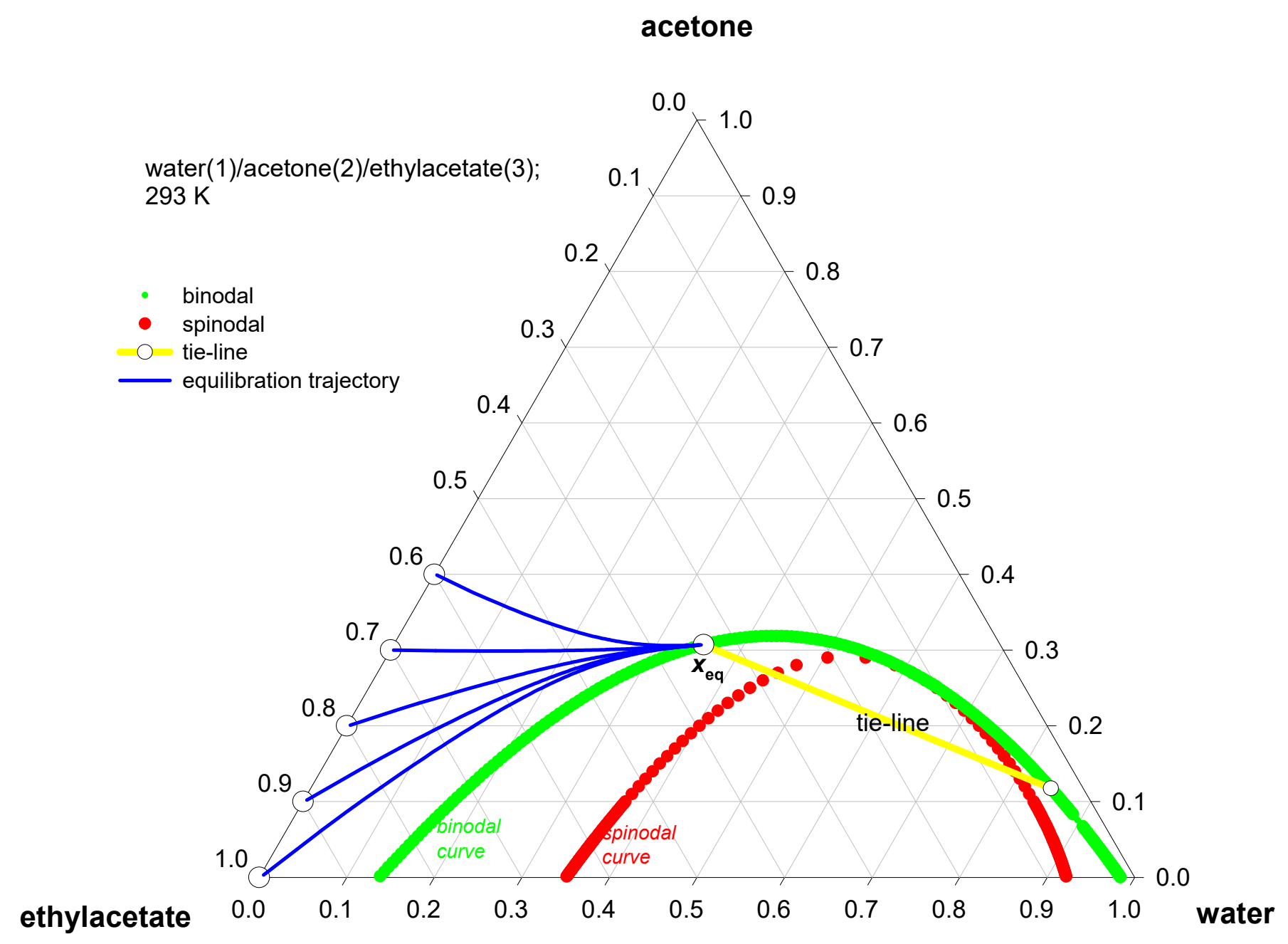




\section{Water/Caprolactam/Toluene equilibratioih s10}
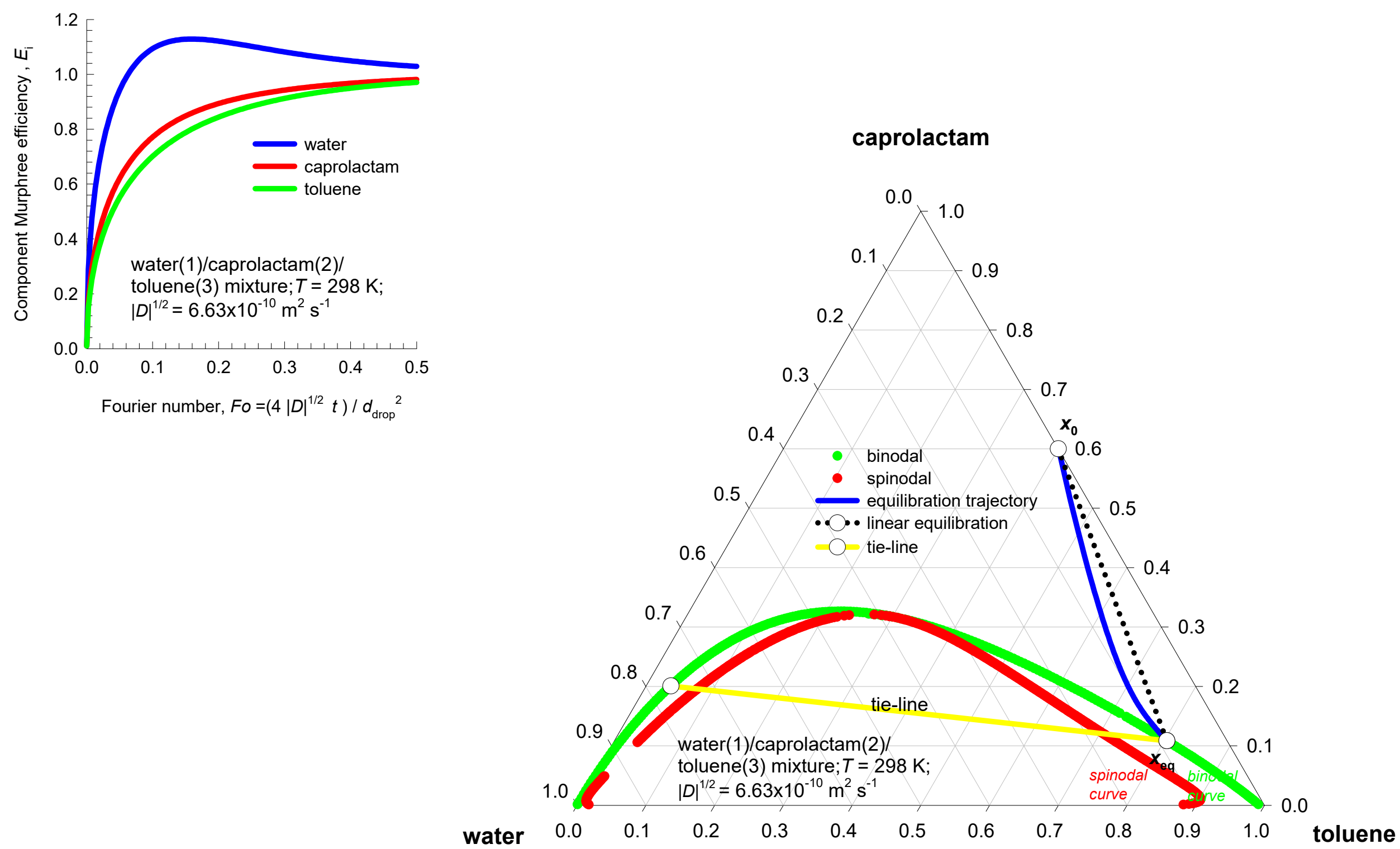


\section{Water/Caprolactam/Toluene equilibratioîi. $\$ 11$}

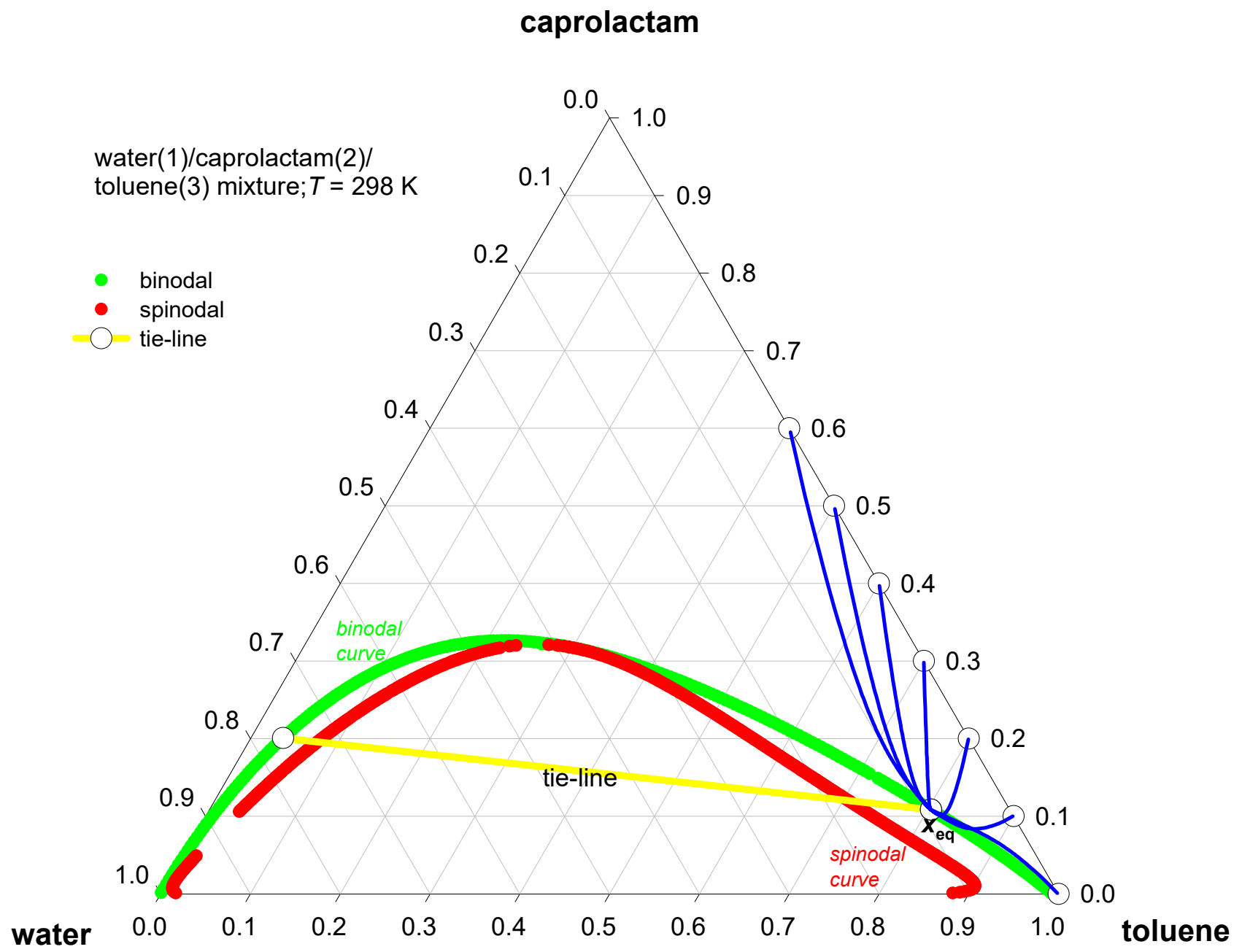




\section{Water/acetic acid/isophorone equilibration}

Fig. S12
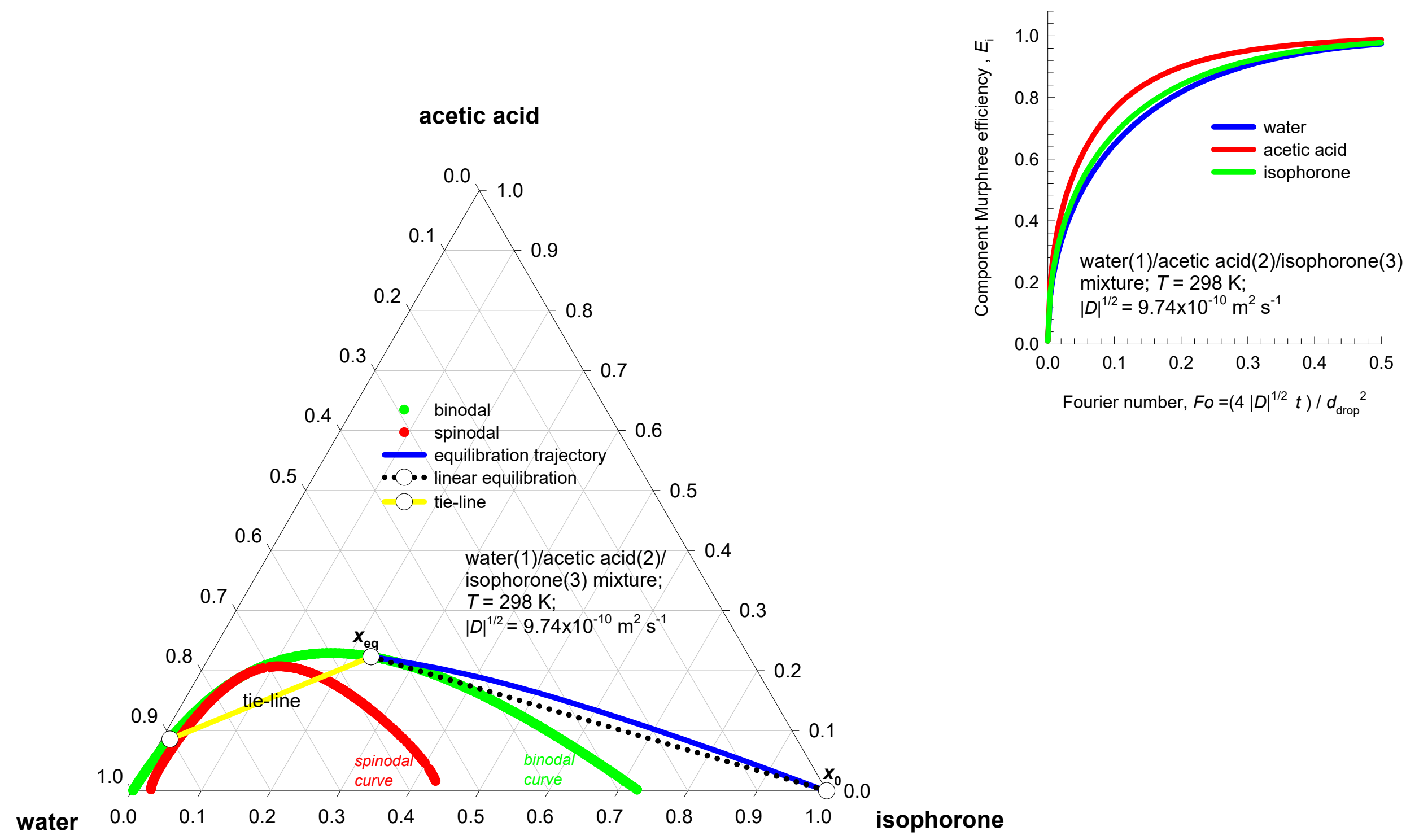


\section{Water/acetic acid/isophorone equilibration}

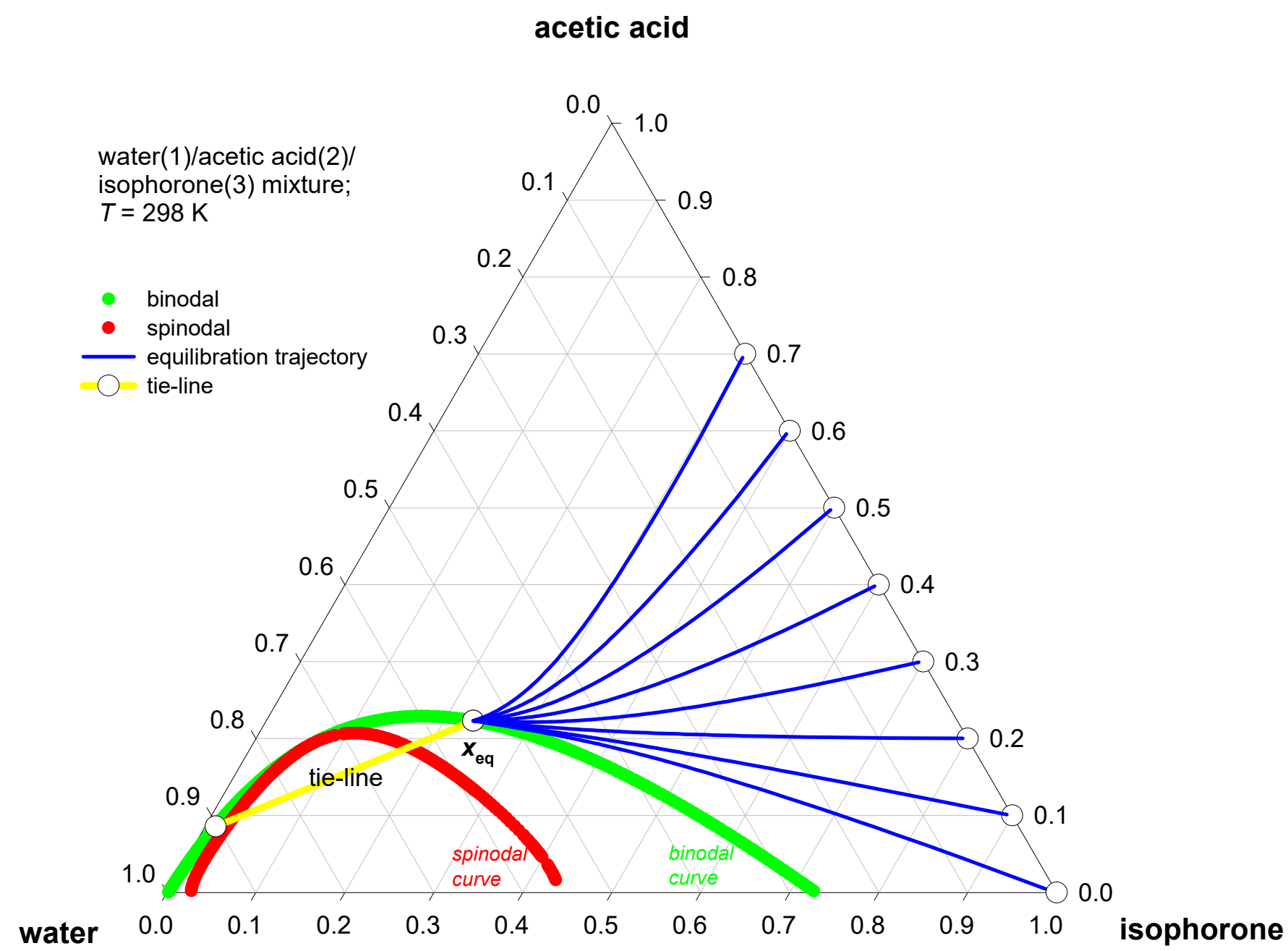


Fig. S14

\section{Water/acetic acid/MTBE equilibration}

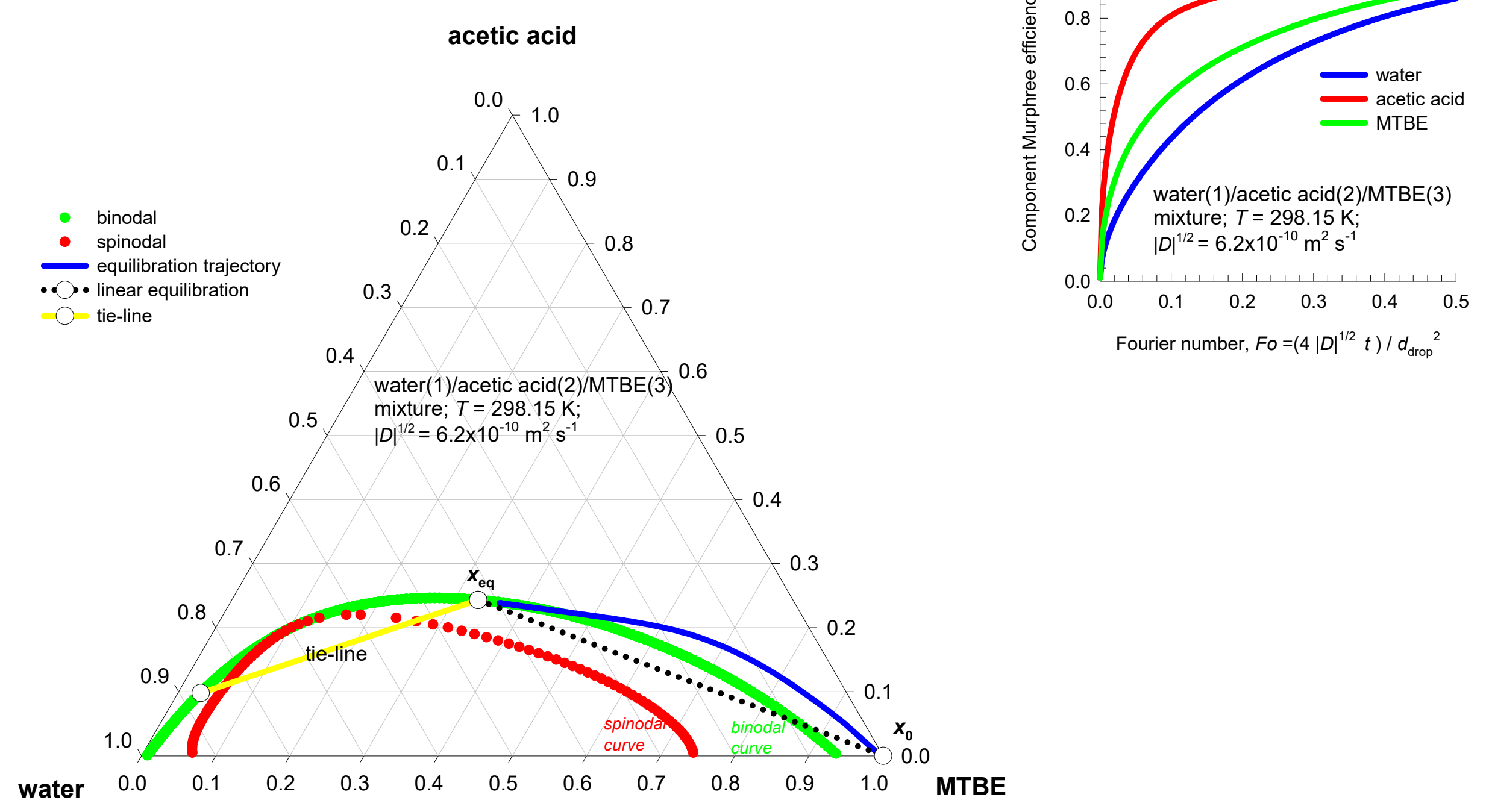




\section{Water/acetic acid/MTBE equilibration}

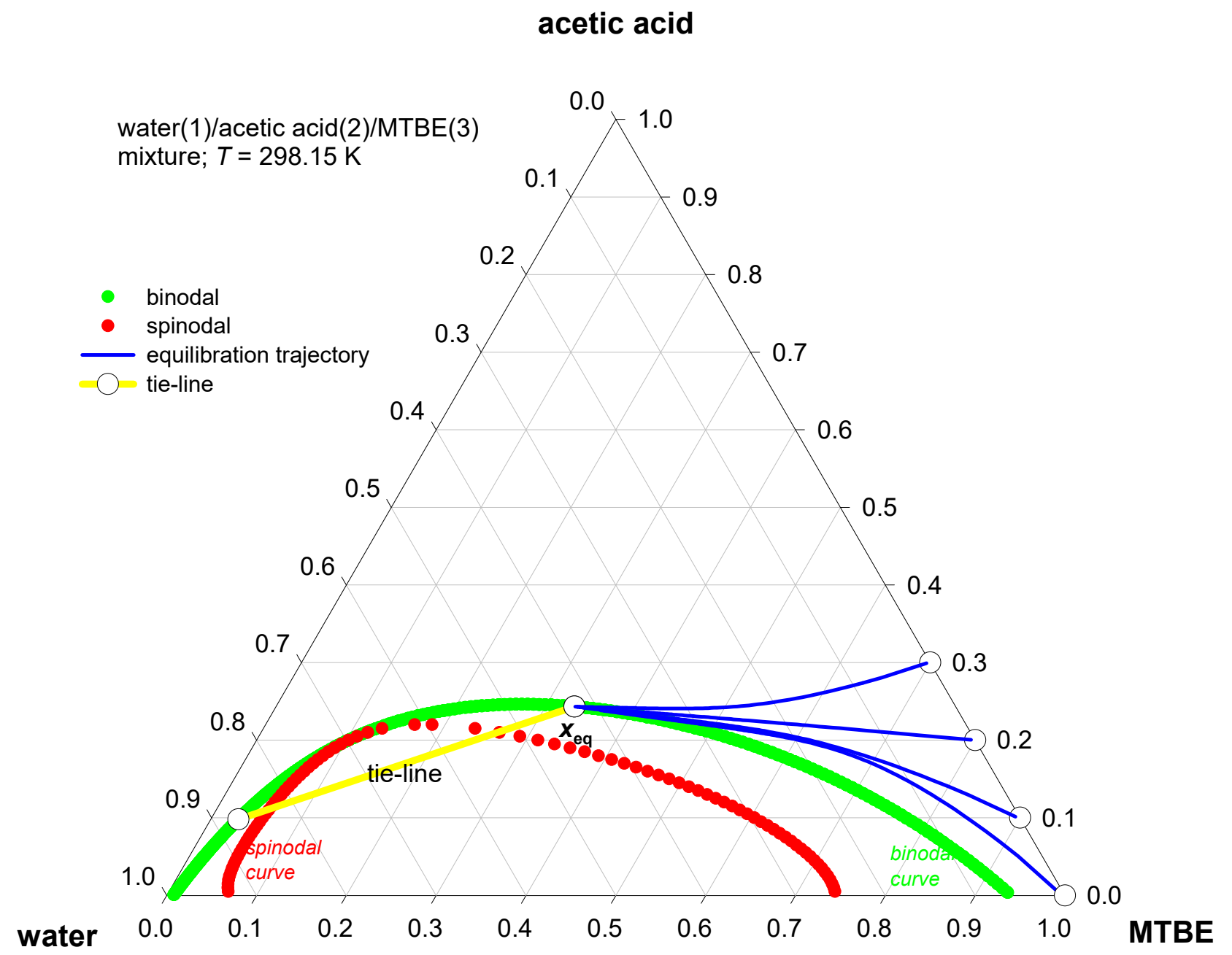




\section{[omim][Cl]/ethanol/TAEE equilibration ${ }^{\text {Fig. S16 }}$}
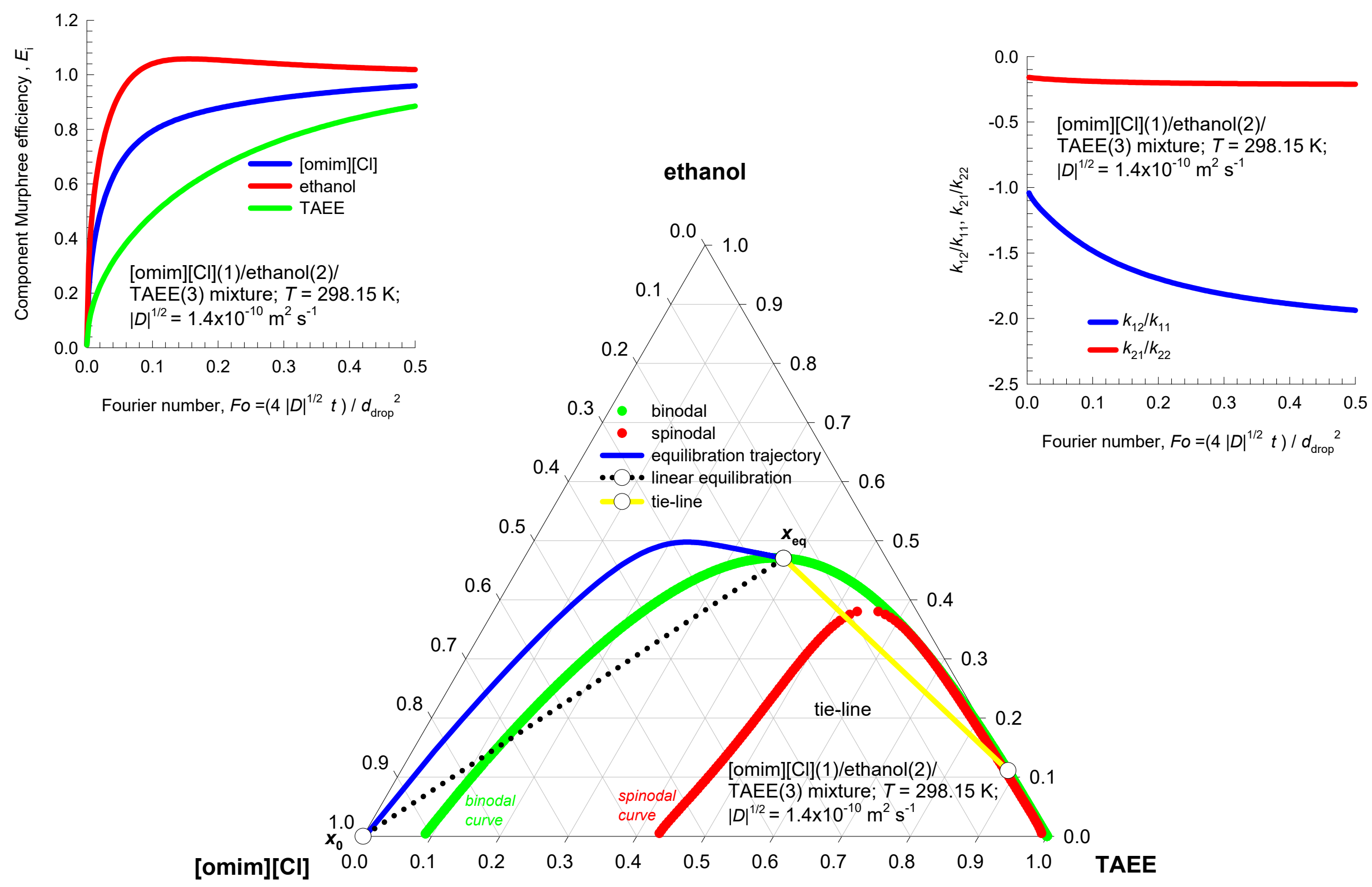


\section{[omim][Cl]/ethanol/TAEE equilibration ${ }^{\text {Fig. s17 }}$}
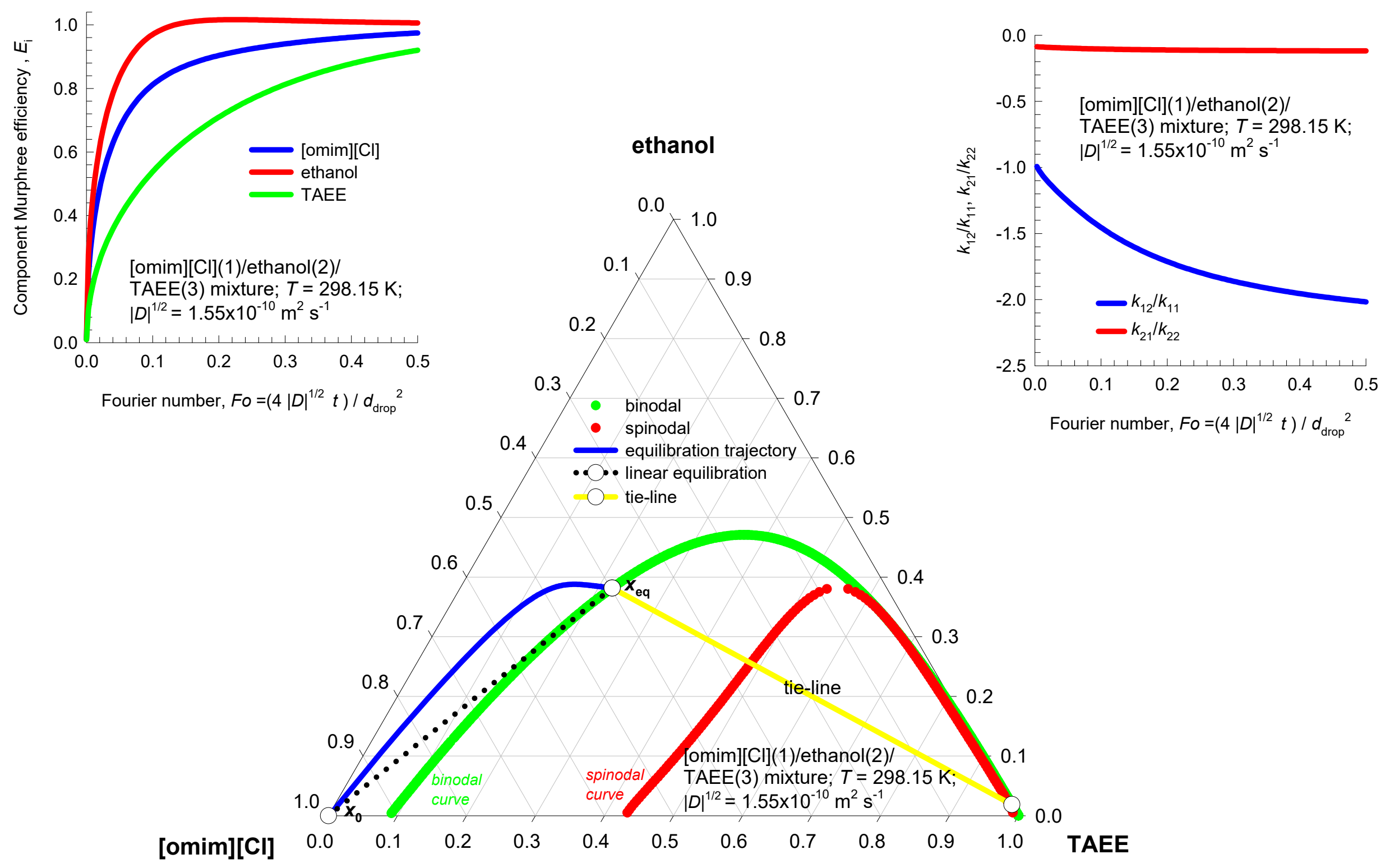


\section{[omim][Cl]/ethanol/TAEE equilibration ${ }^{\text {Fig. s18 }}$}

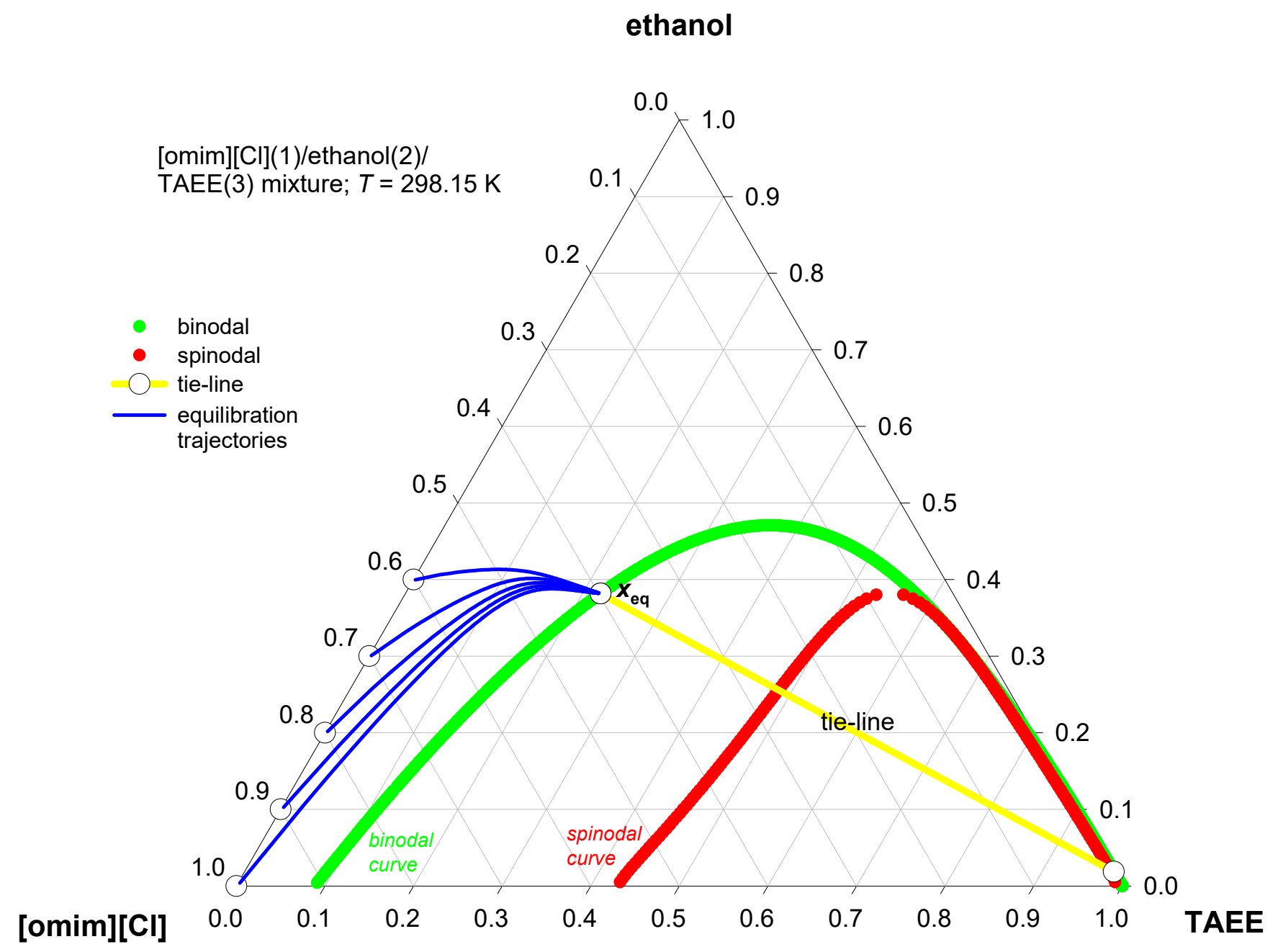




\section{[bmim][TfO]/ethanol/TAEE equilibration ${ }^{\text {ig. s19 }}$}
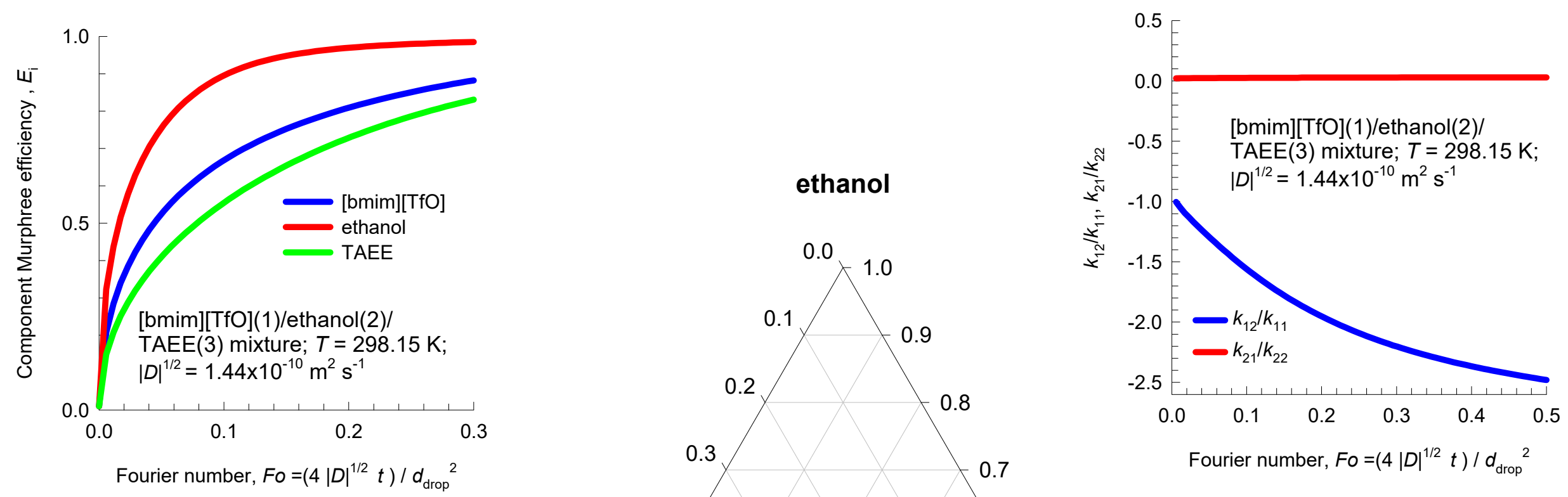

[bmim][TfO]

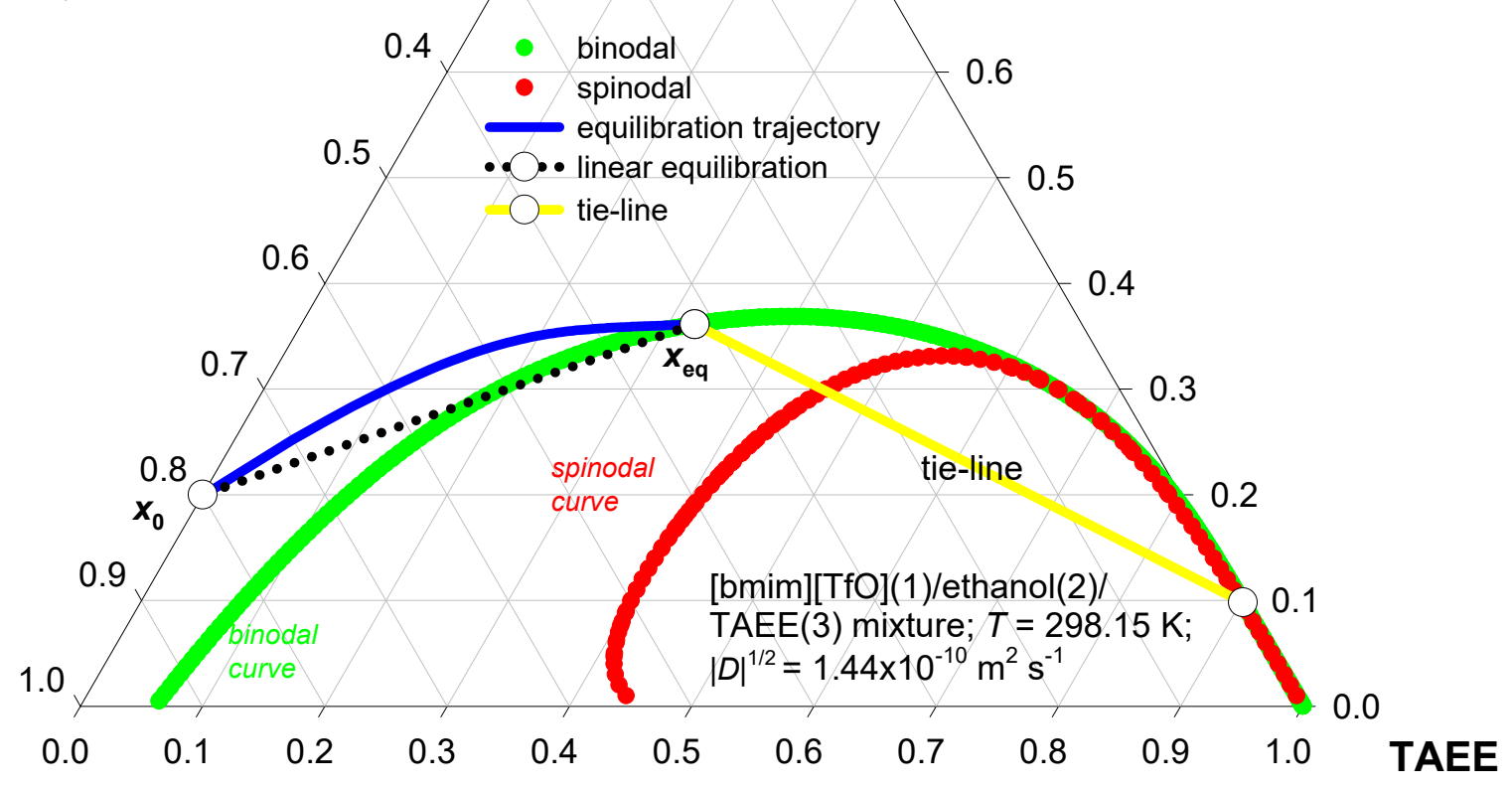




\section{[bmim][TfO]/ethanol/TAEE equilibration ${ }^{\text {fig. s20 }}$}

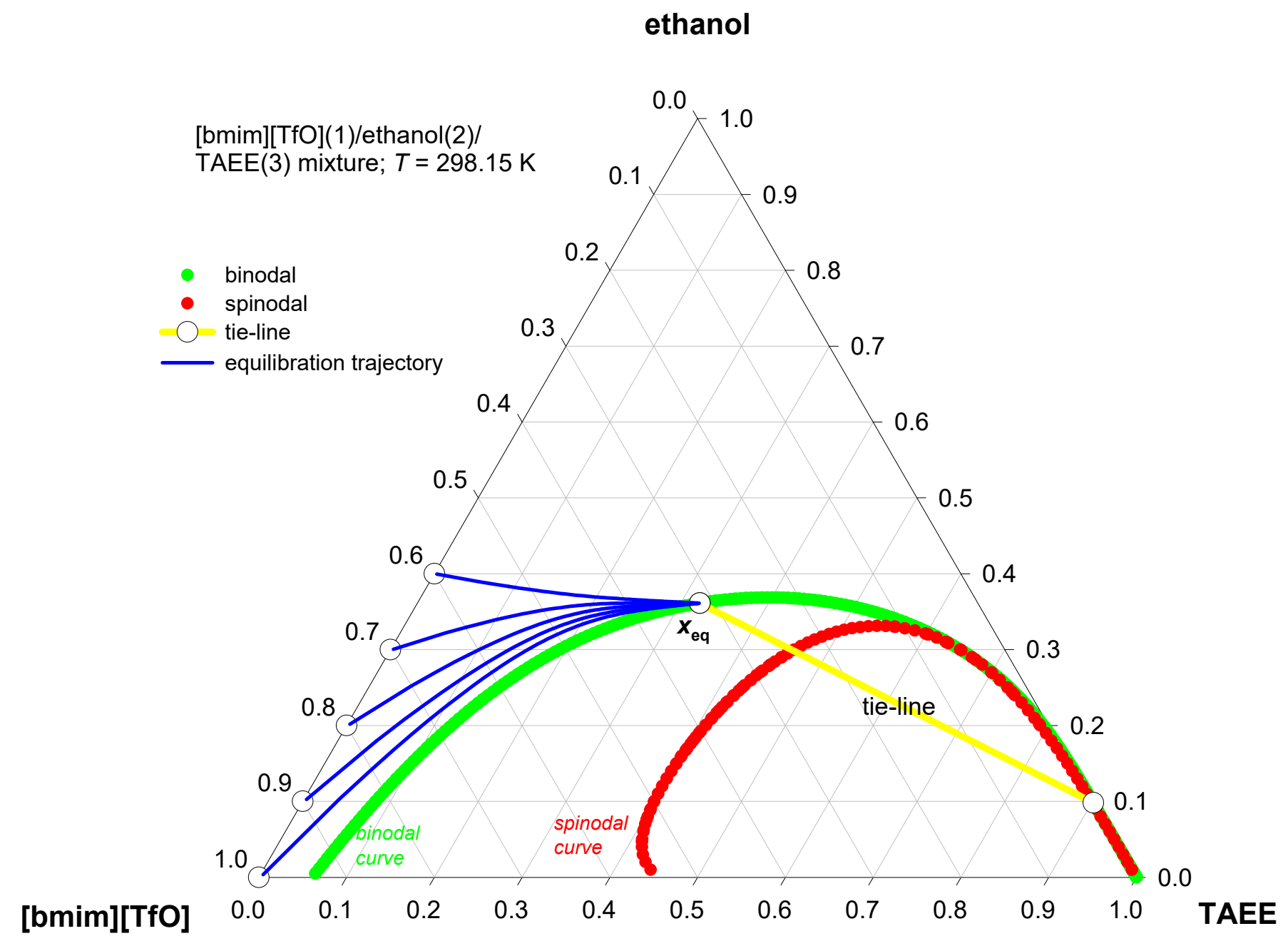




\section{Water/Ethanol/Cyclohexane equilibratiofiq. ${ }^{221}$}
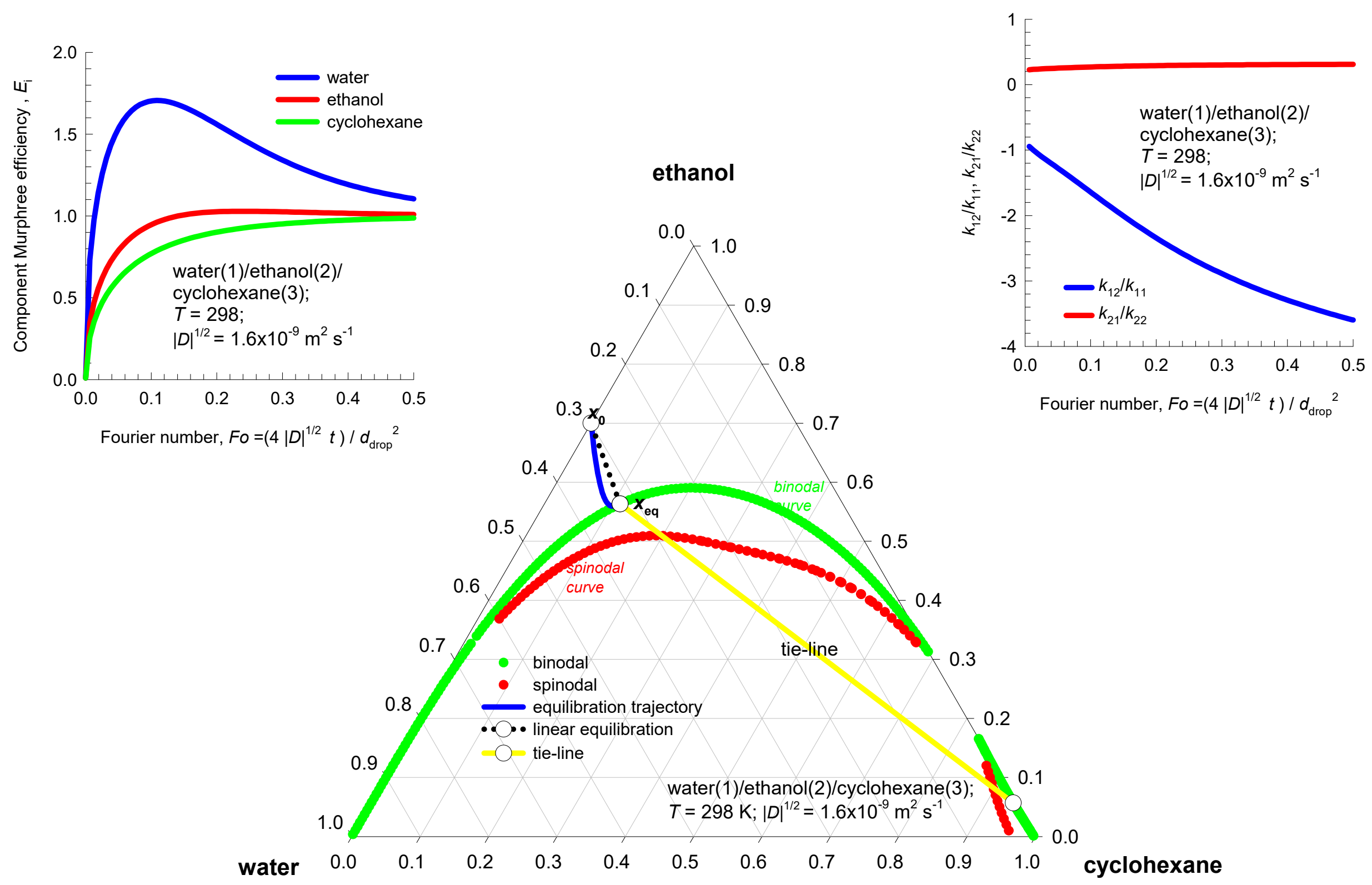


\section{Water/Ethanol/Cyclohexane equilibratiofig. s22}

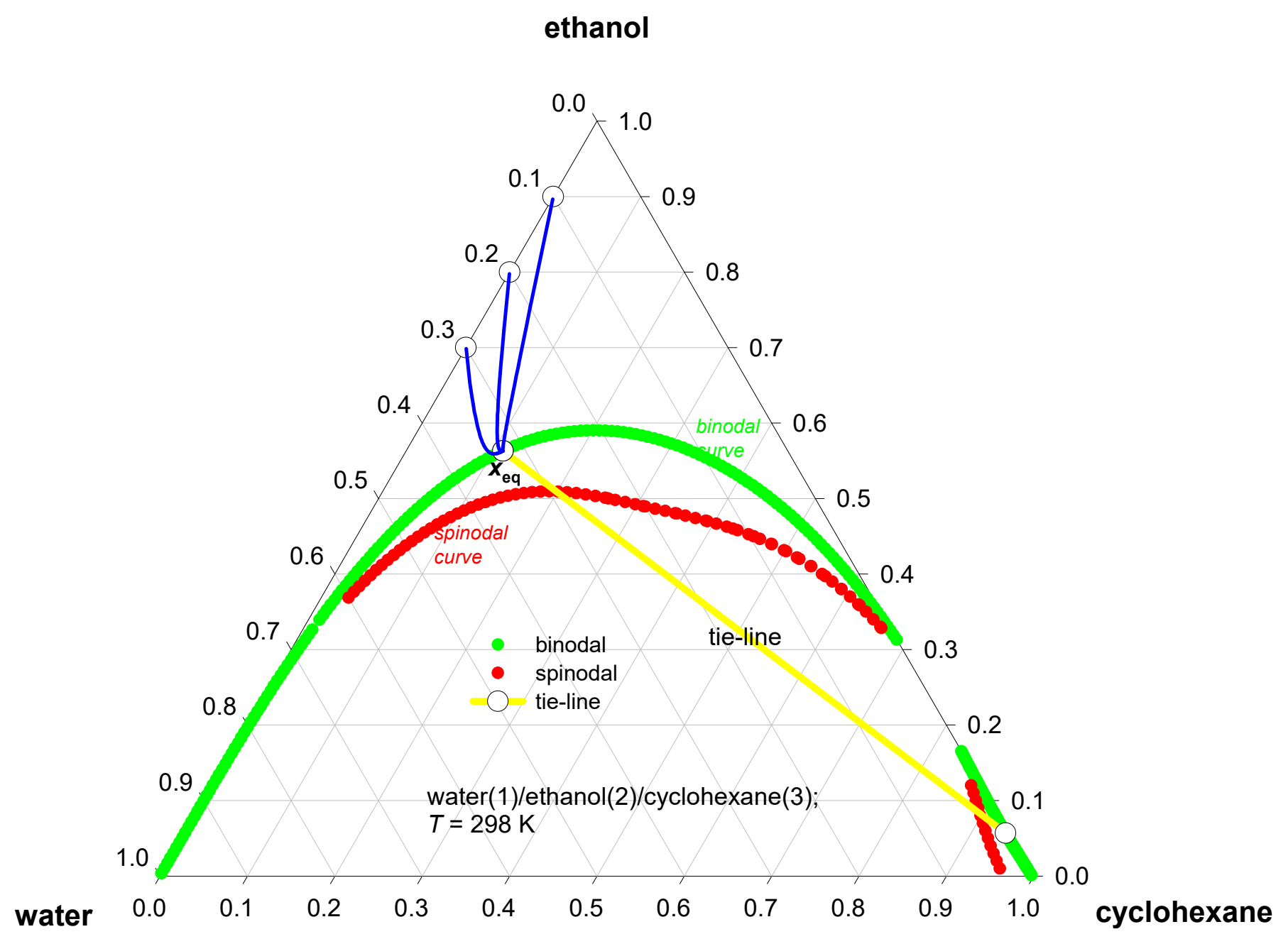




\section{Toluene/ethanol/water equilibration}

Fig. S23

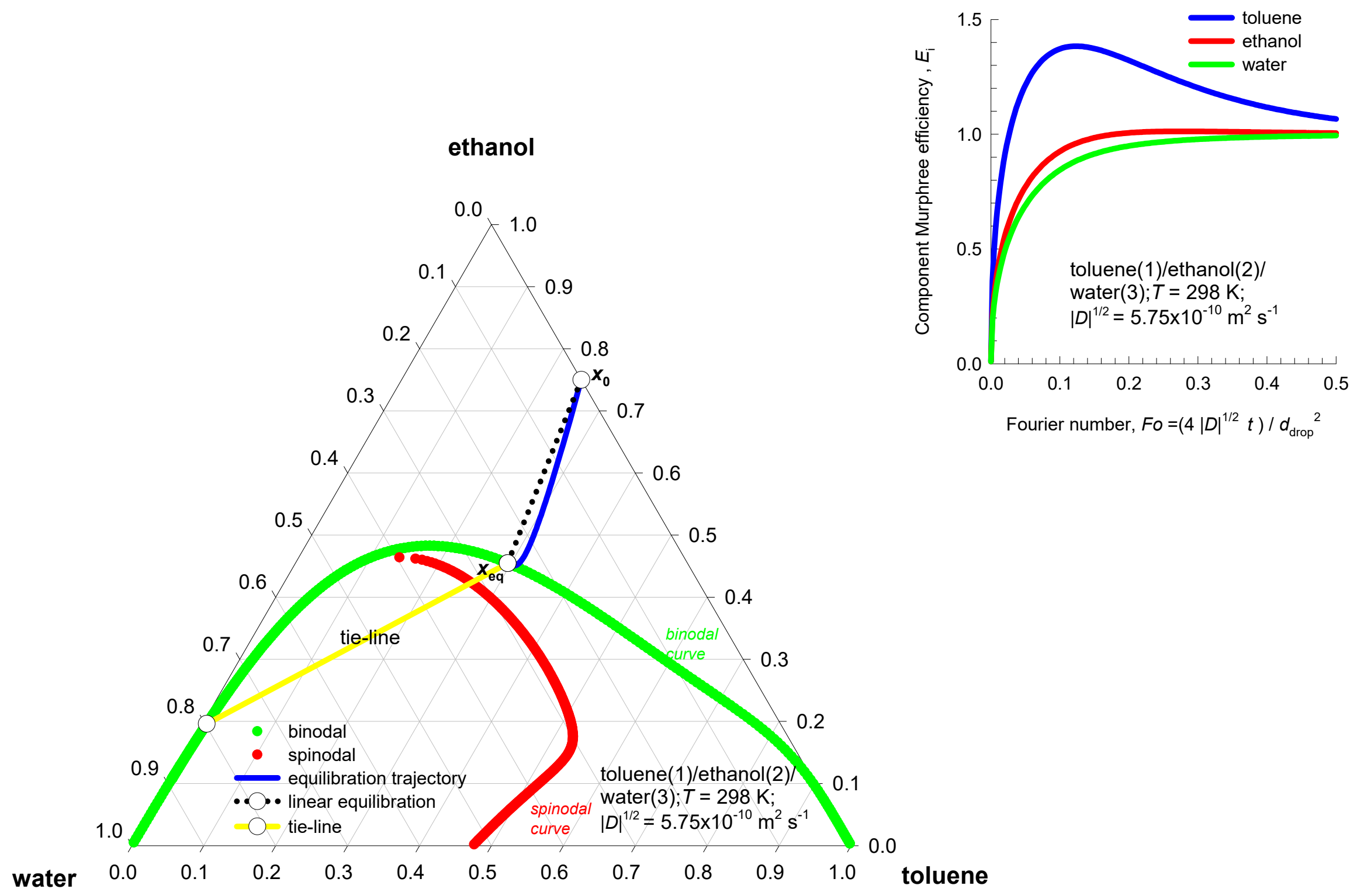




\section{Toluene/ethanol/water equilibration}

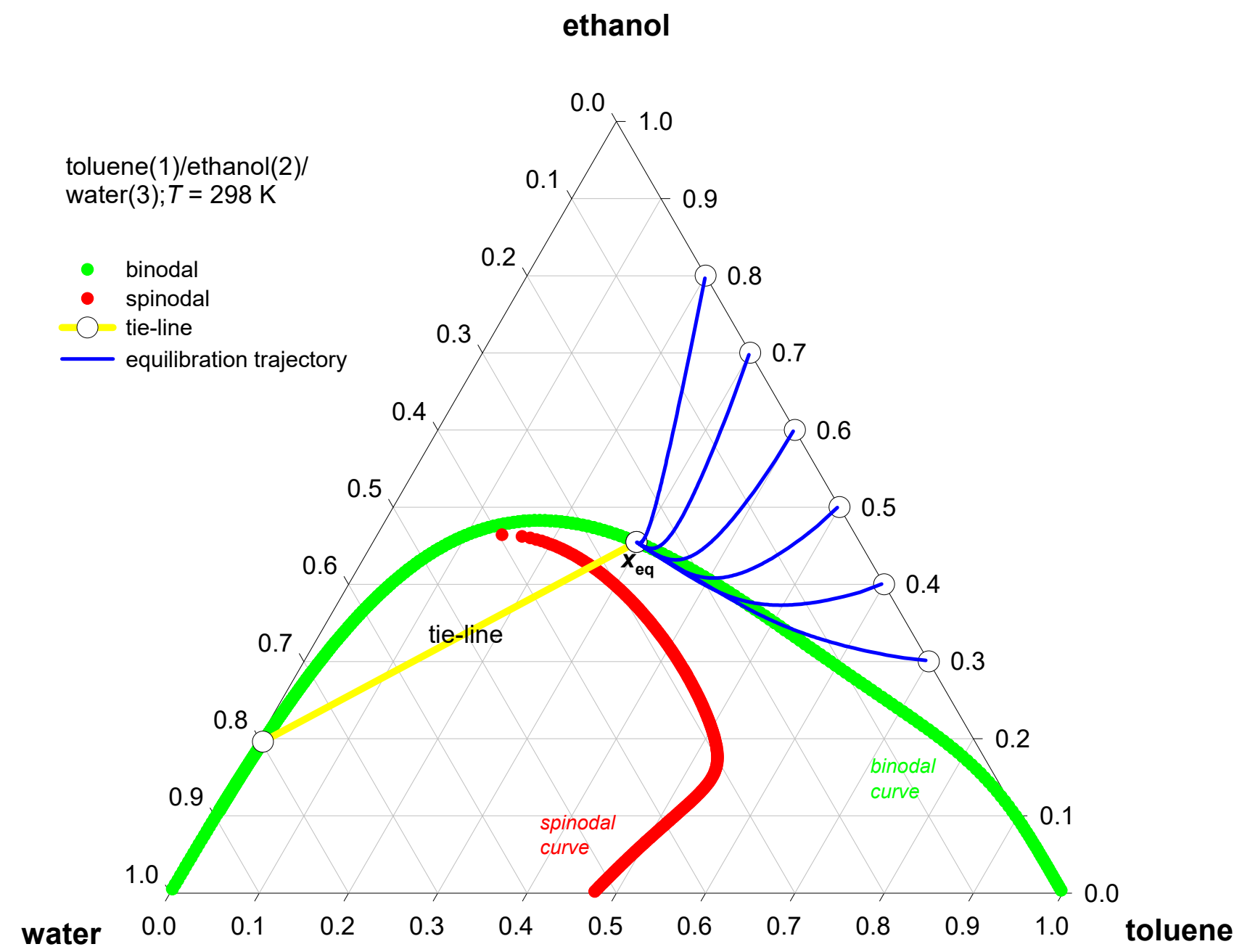




\section{Water/Acetone/Toluene equilibration Fig. S25}
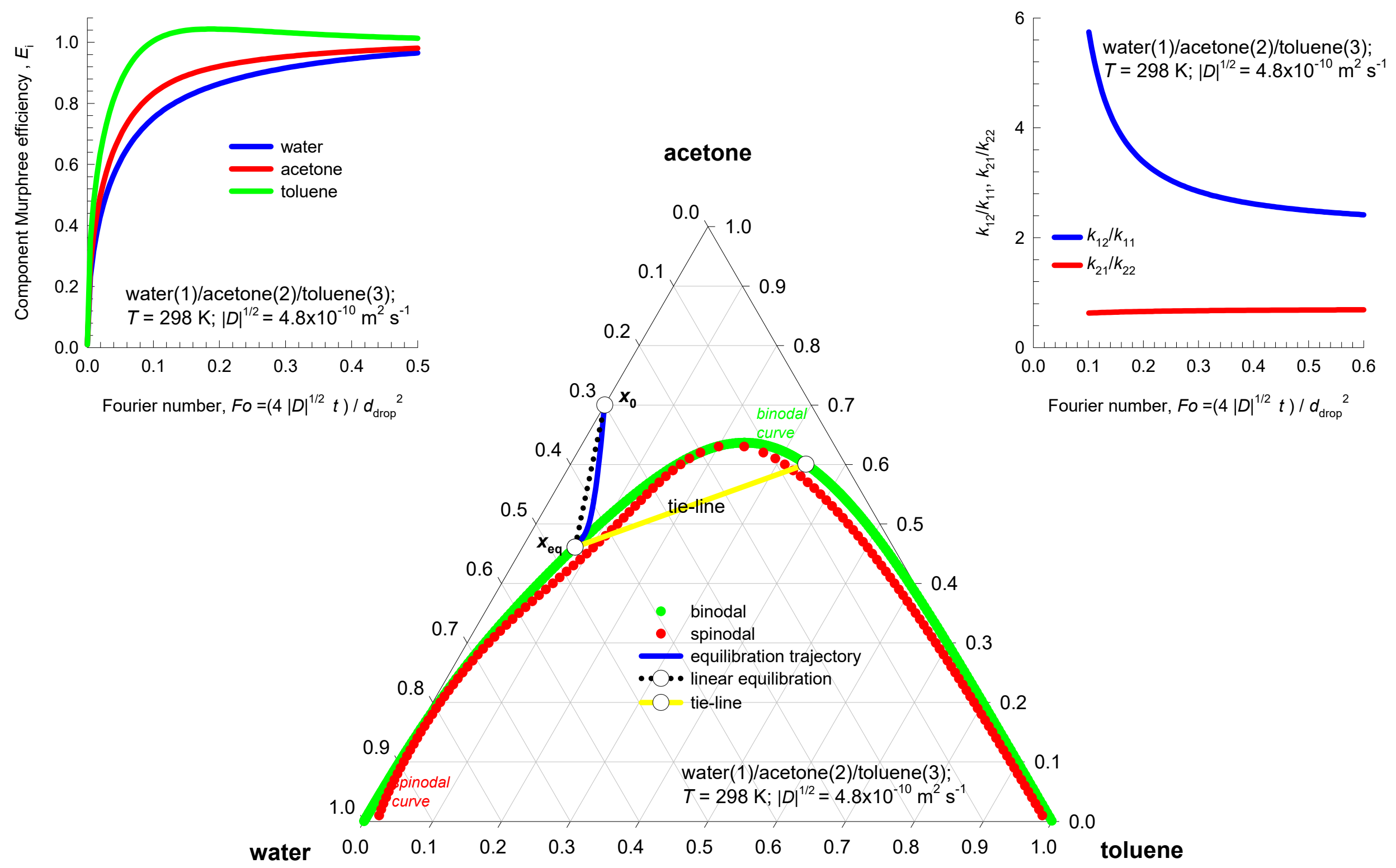


\section{Water/Acetone/Toluene equilibration ${ }^{\text {Fig. }}$ s26}

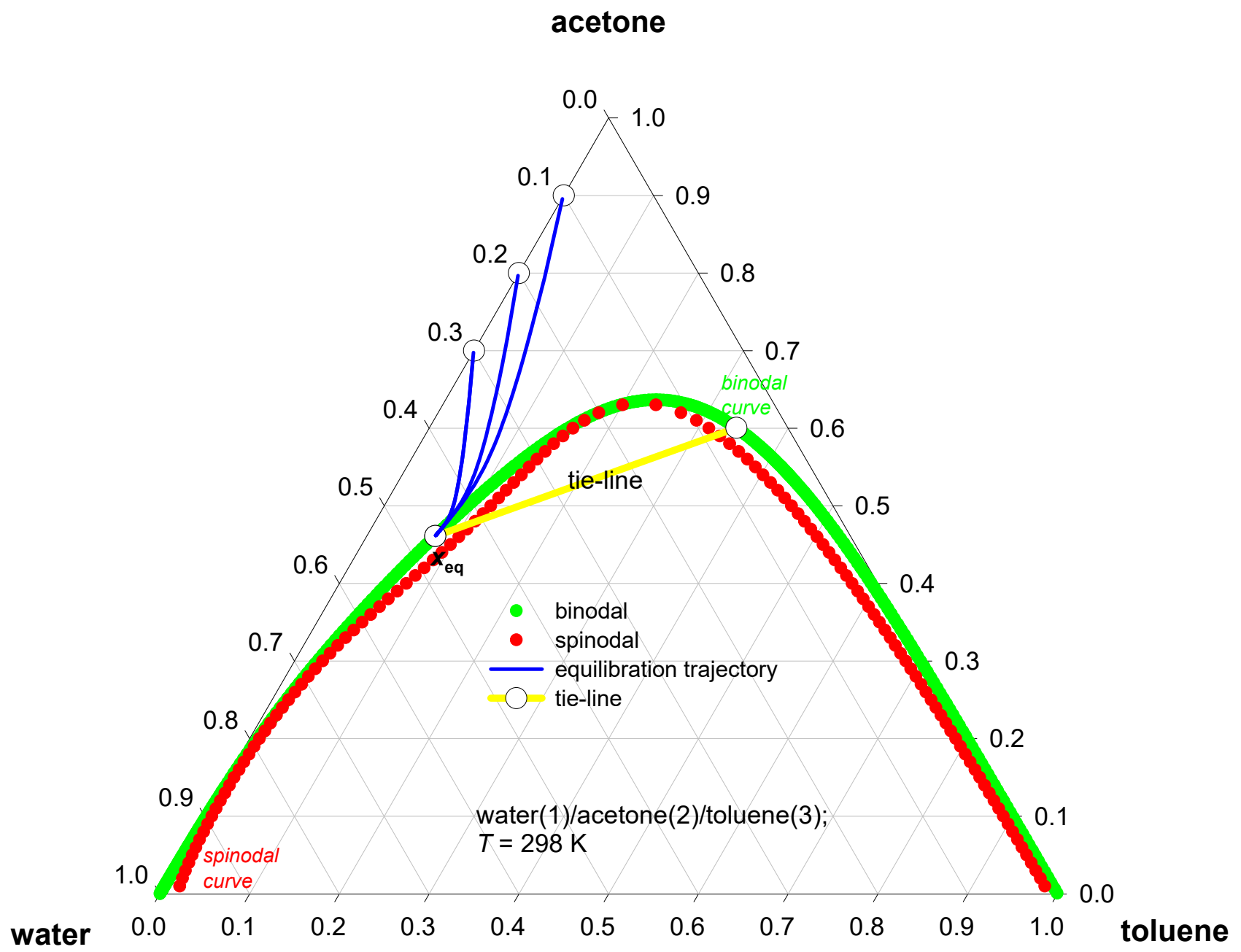


Fig. S27

\section{Vapor/liquid contacting on distillation tray}
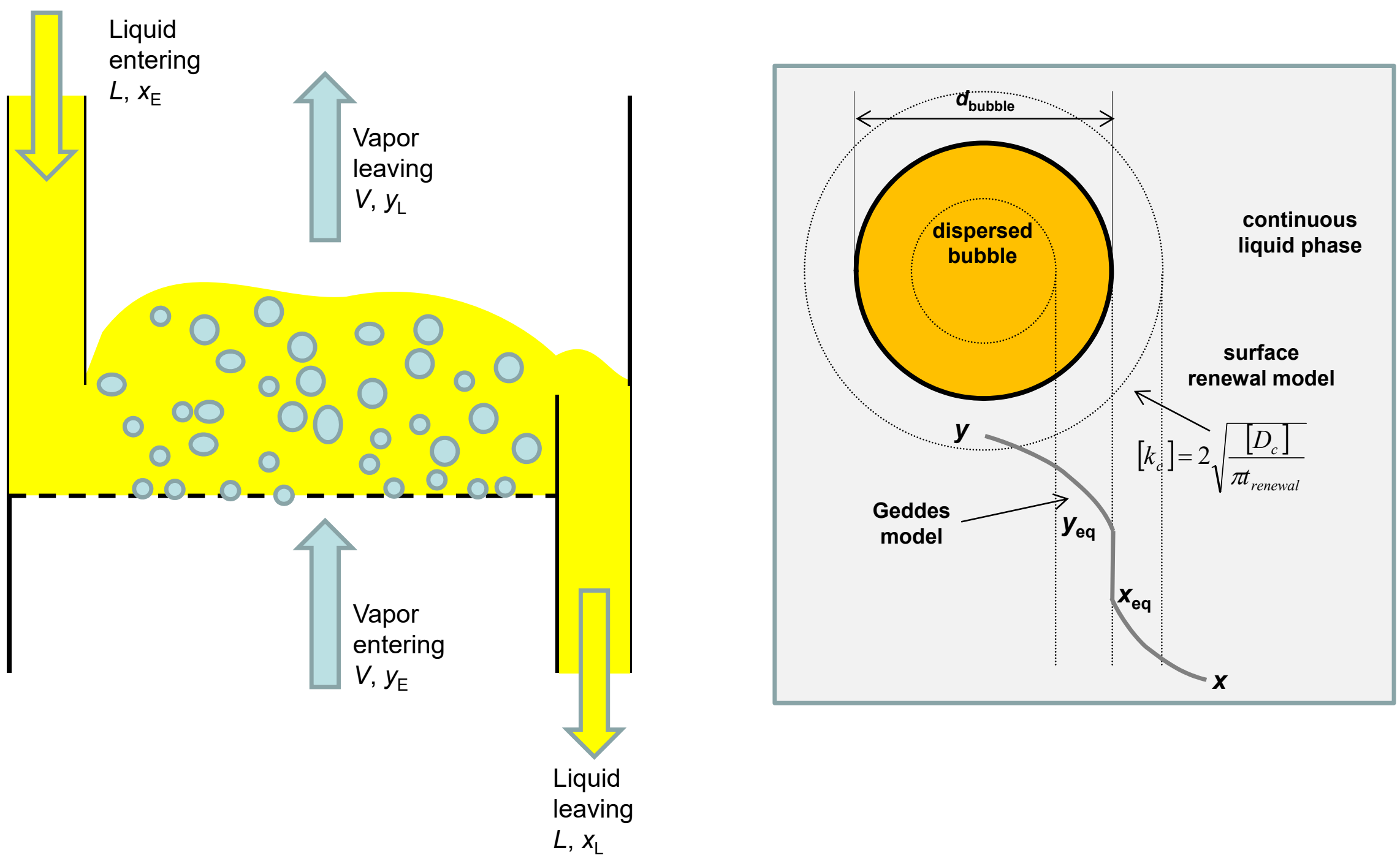

leaving 


\section{Levy rules for boundary crossing}

Fig. S28
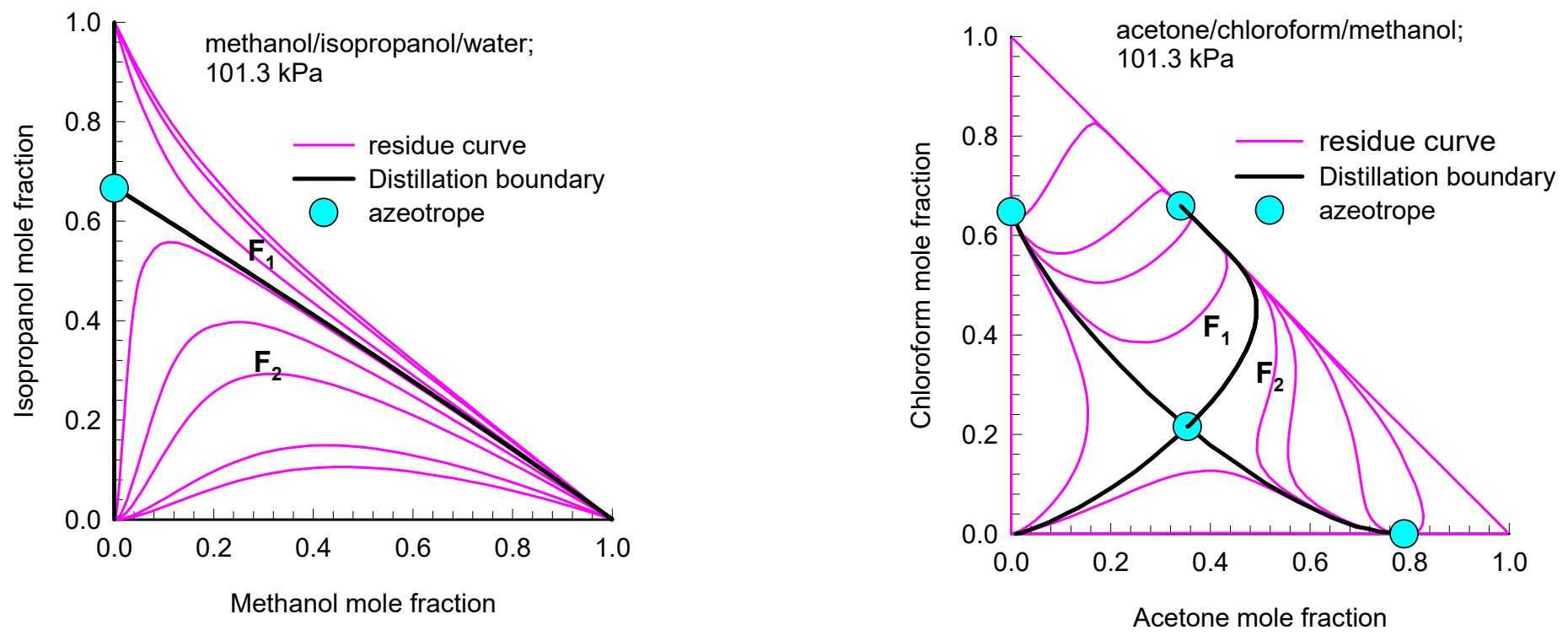


\section{Springer experimental set-up}

Fig. S29

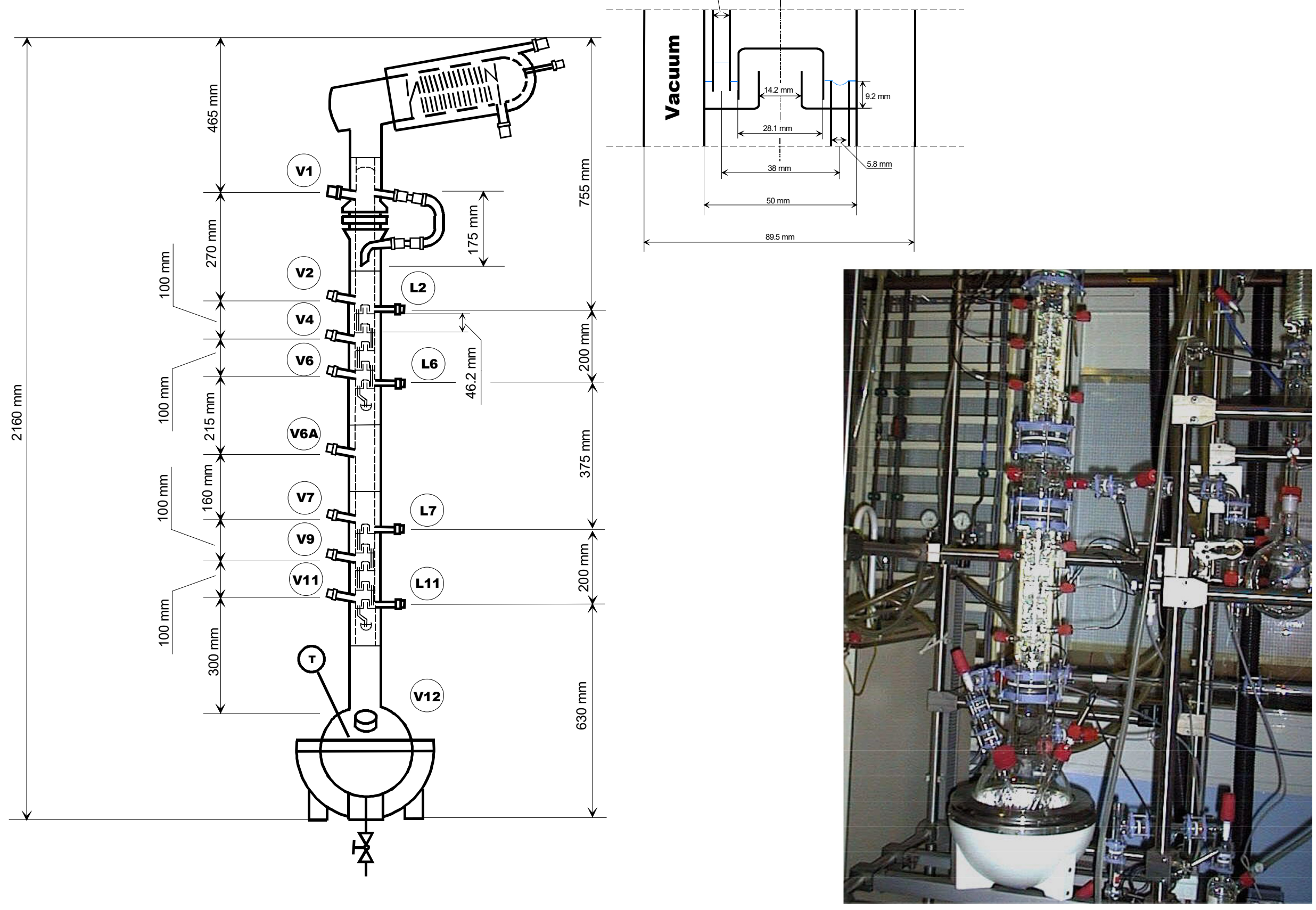




\section{Water/Ethanol/Acetone Distillation}

Fig. S30

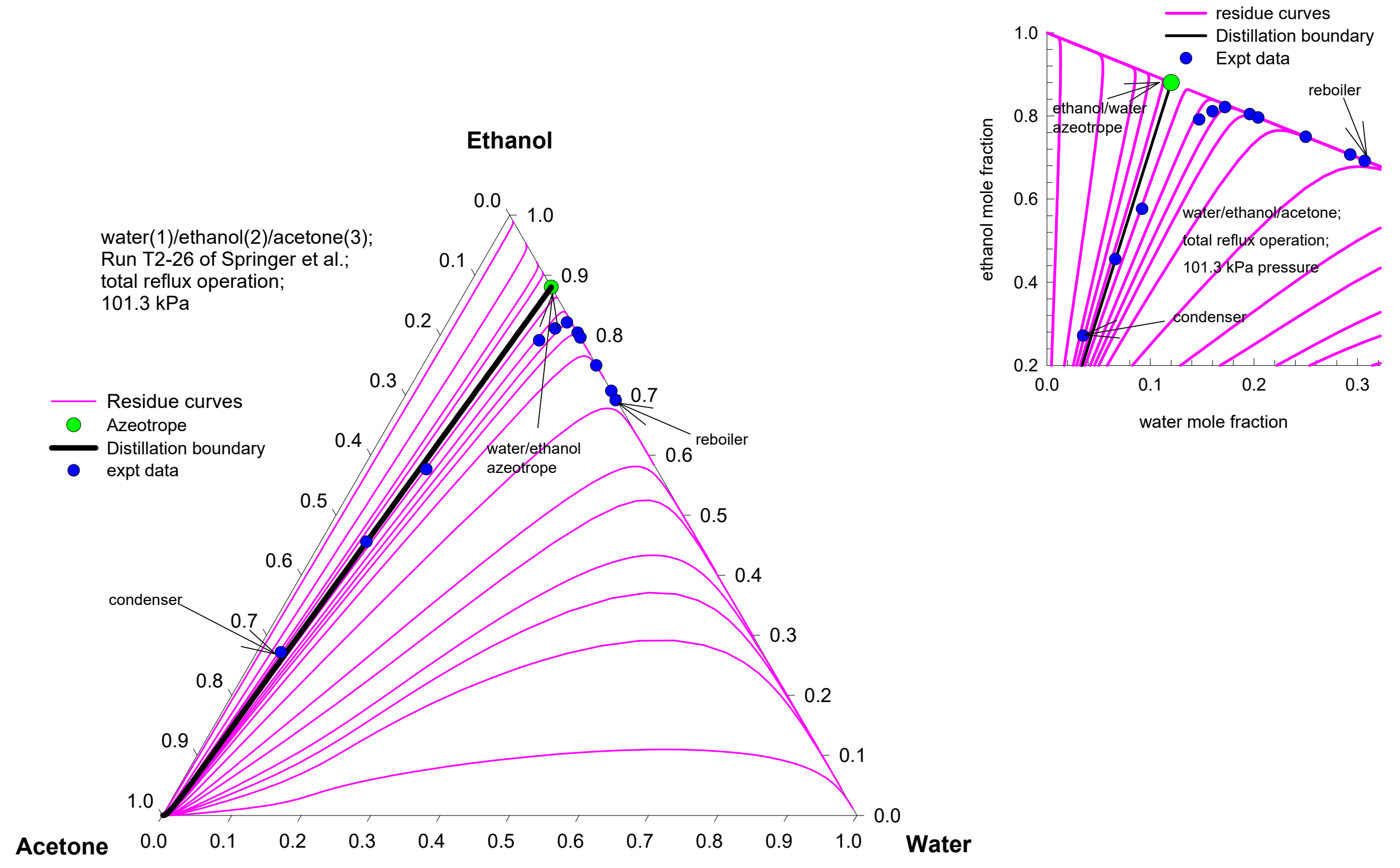




\section{Murphree component efficiencies}

(a)

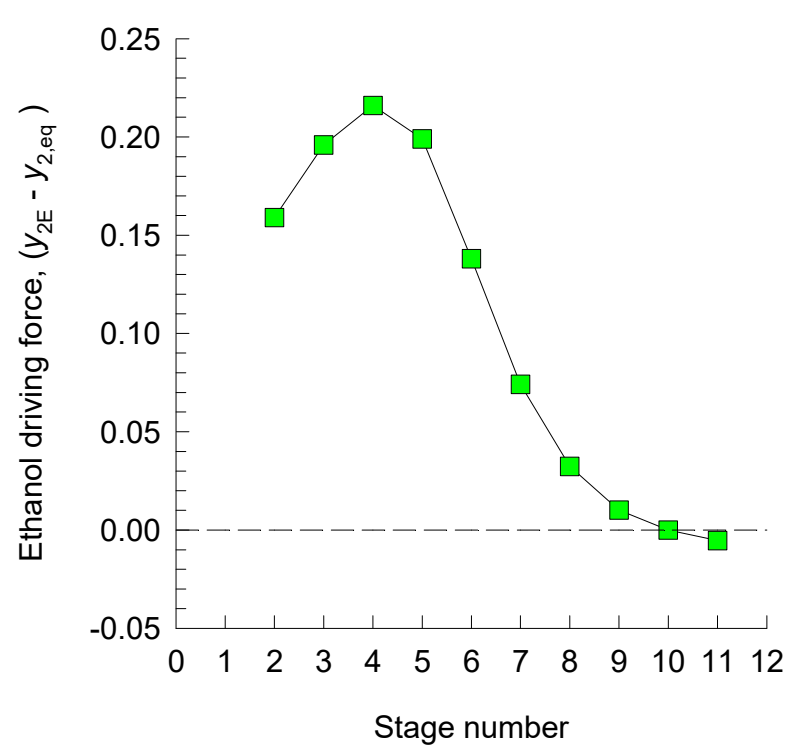

(b)

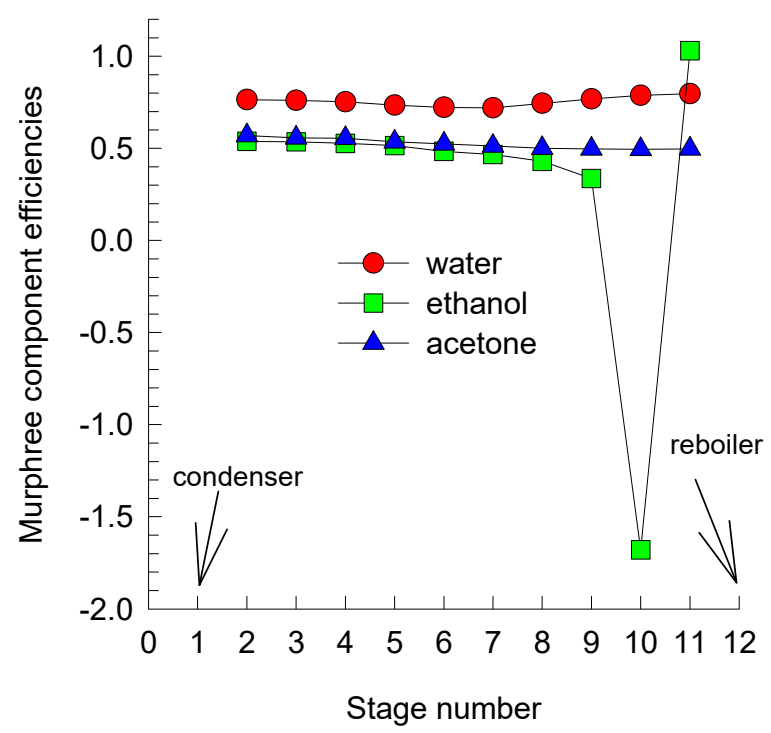




\section{Water/Ethanol/Methanol Distillation}

Fig. S32
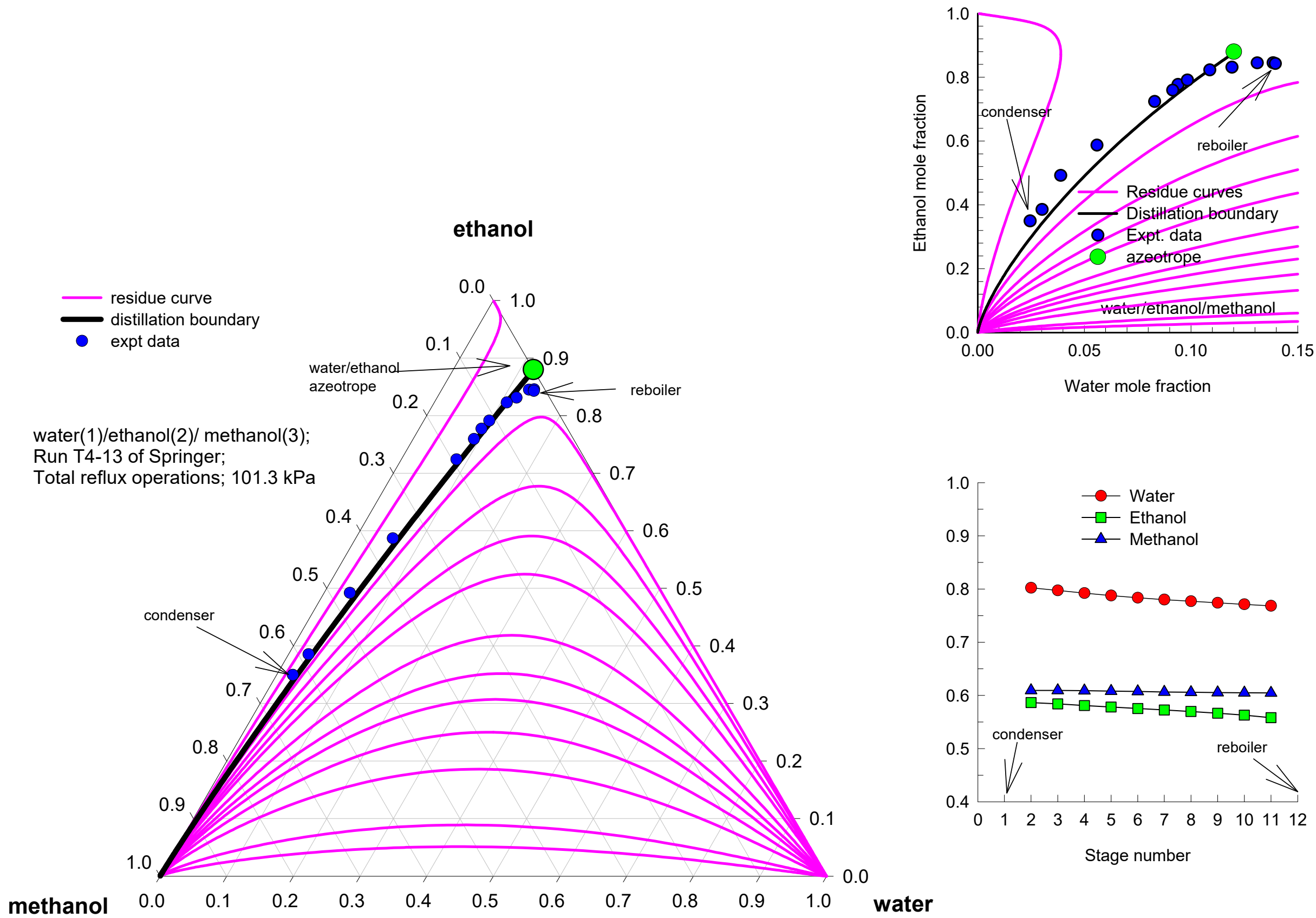

Run T4-13 of Springer;

Total reflux operations; $101.3 \mathrm{kPa}$

methanol 


\section{Water/Ethanol/Methylacetate Distillation ${ }^{\text {fig. }}{ }^{\text {\$33 }}$}

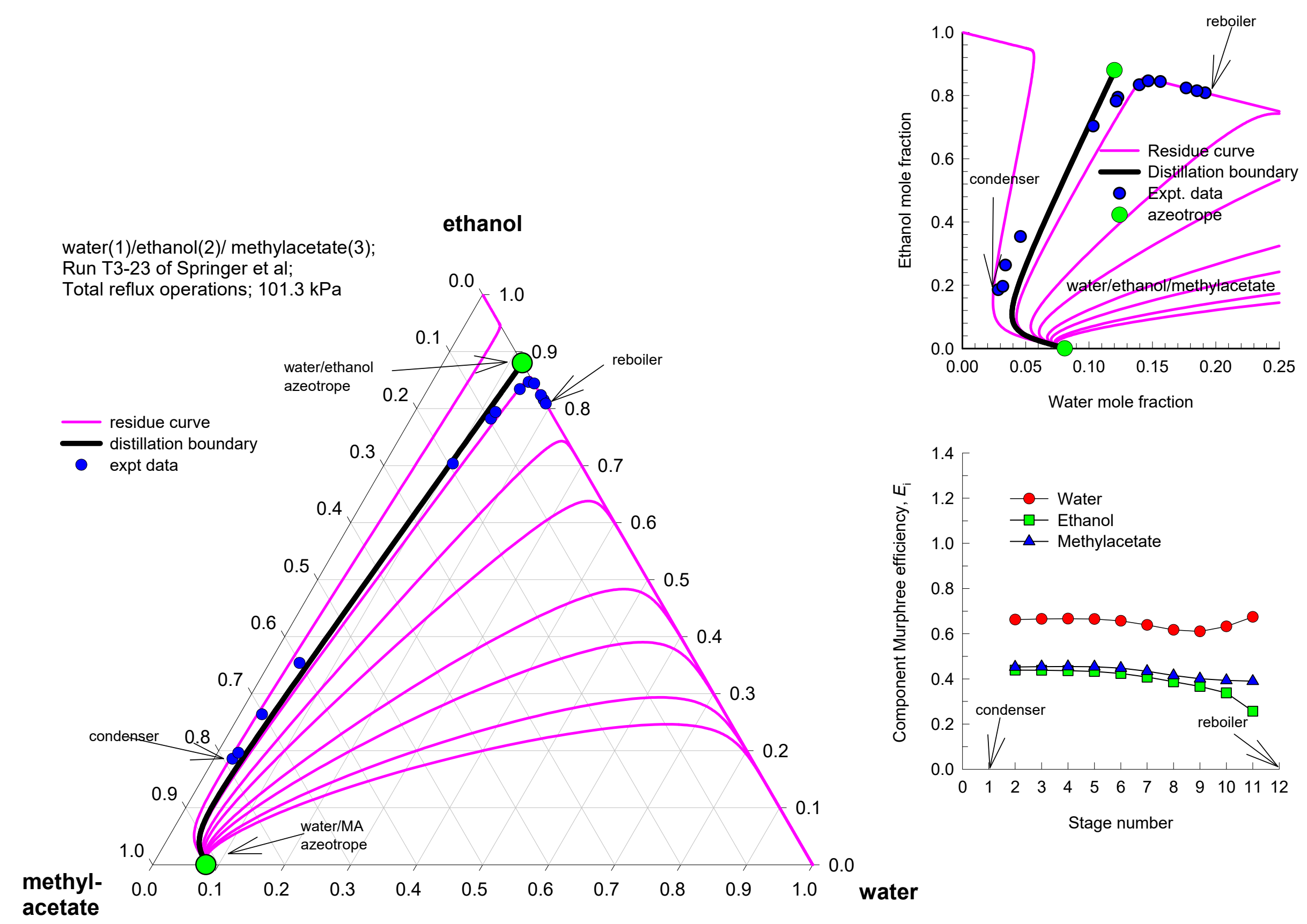




\section{Water/Ethanol/Acetone/Methanol Distillation ${ }^{\mathrm{S34}}$}

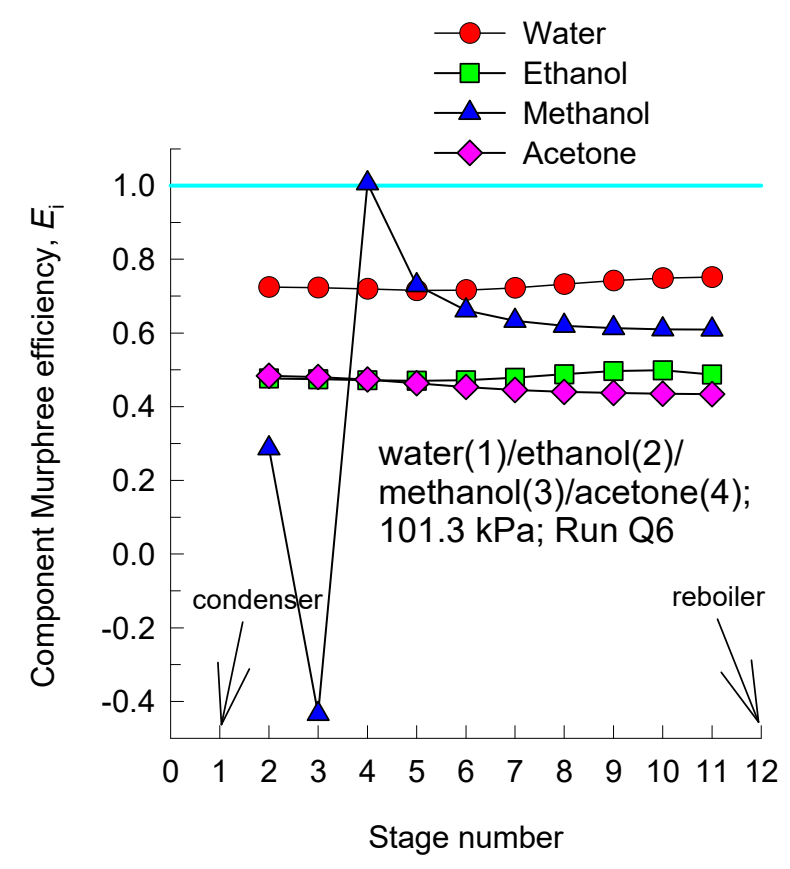

ethanol
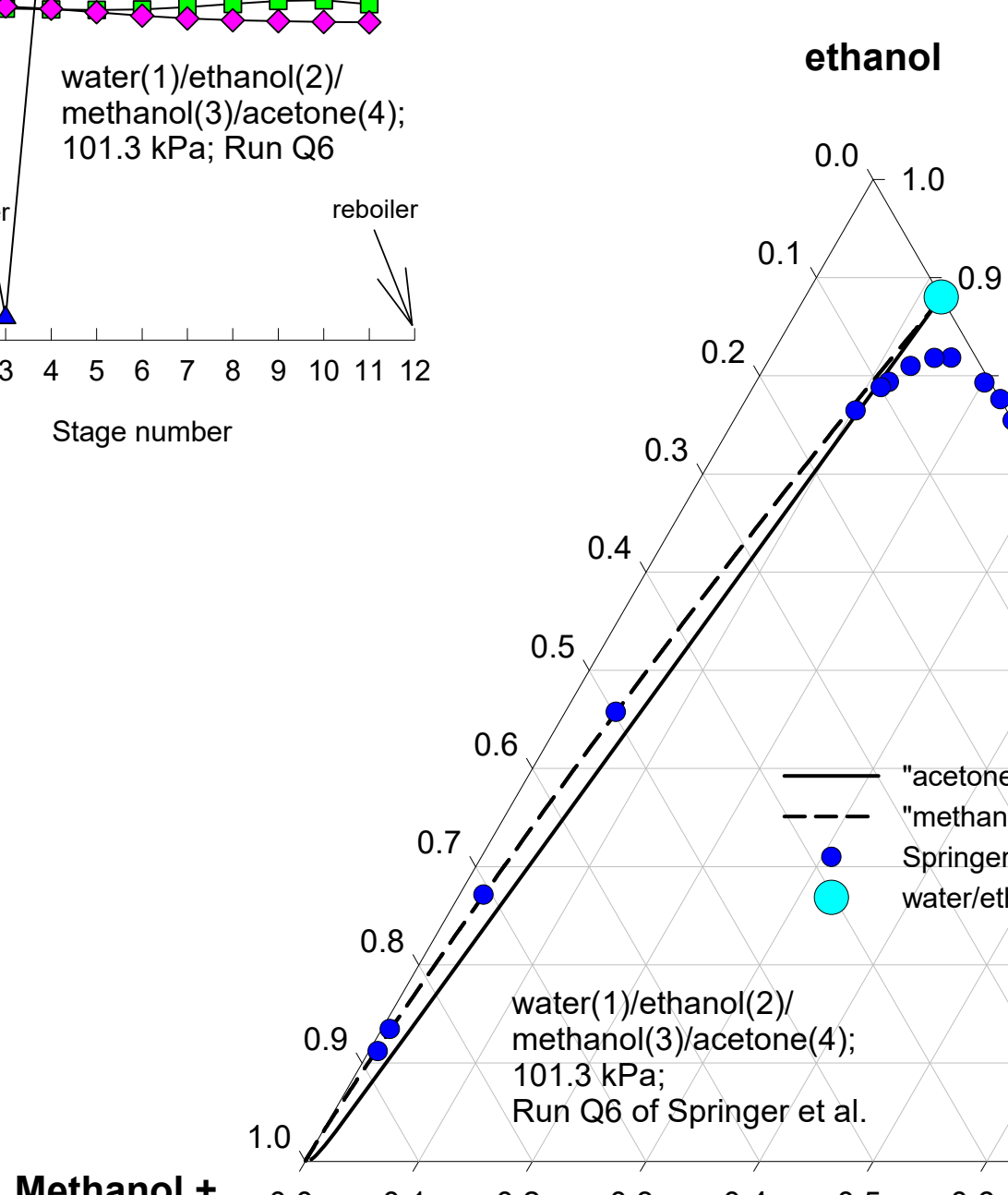

0.0
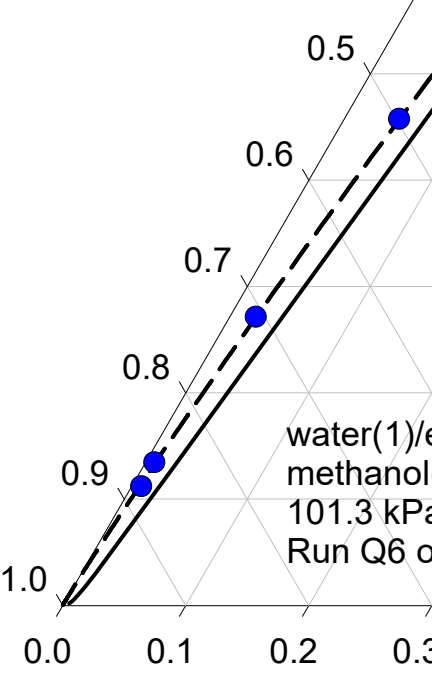

02

0.3

0.5

0.6

\section{Acetone}
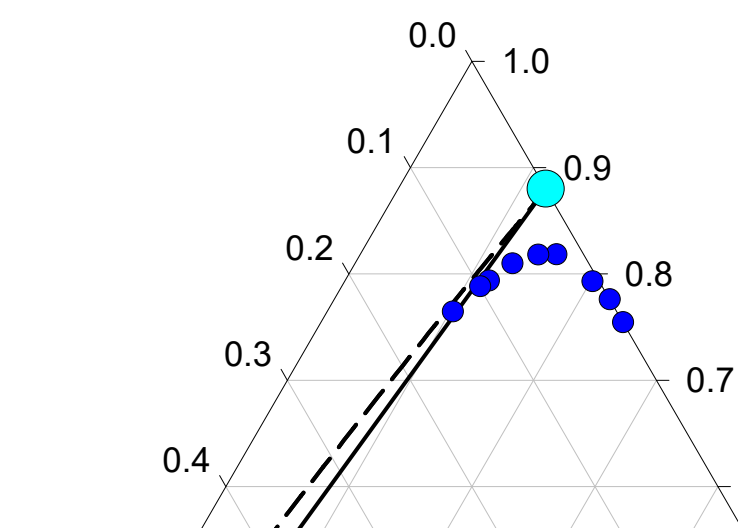

0.5
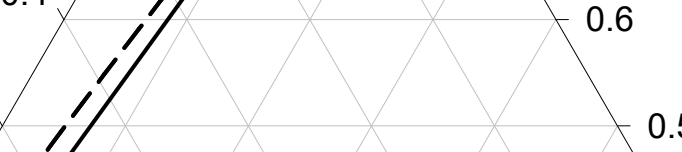

6
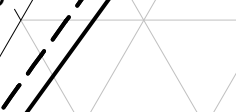

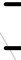

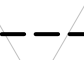

"acetone" boundary "methanol" boundary

- Springer expt dtata water/ethanol azeotrope

water(1)/ethanol(2)/

methanol(3)/acetone(4);

$101.3 \mathrm{kPa}$;

Run Q6 of Springer et al.

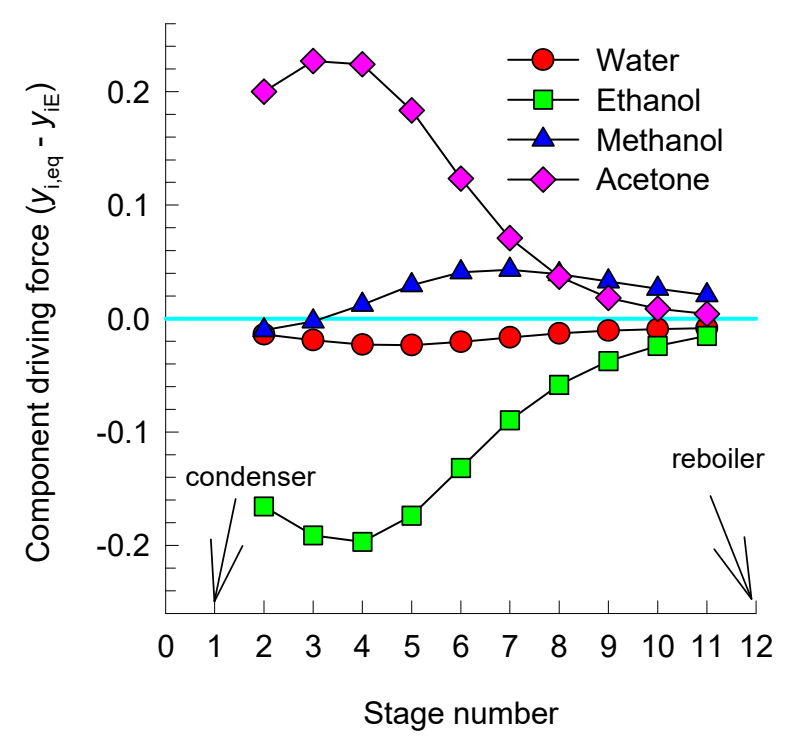

0.4

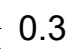

0.7
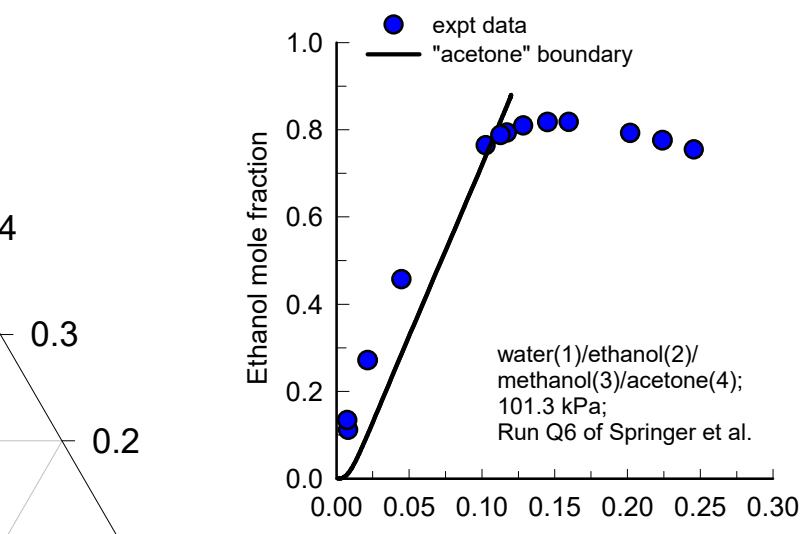
(a)

\section{Water/Ethanol/Acetone Distillation}

(b)

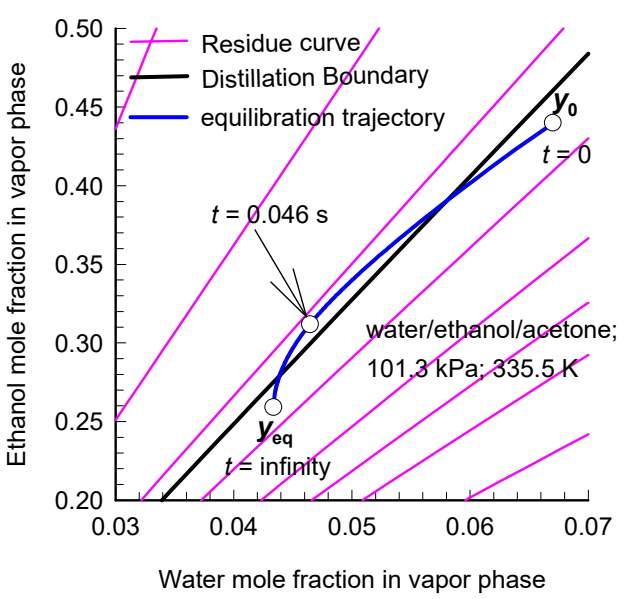

(c)
Distillation Boundary
- Start Compositions
water/ethanol/acetone; total reflux operation;
- NEQ trajectory
$101.3 \mathrm{kPa}$ pressure

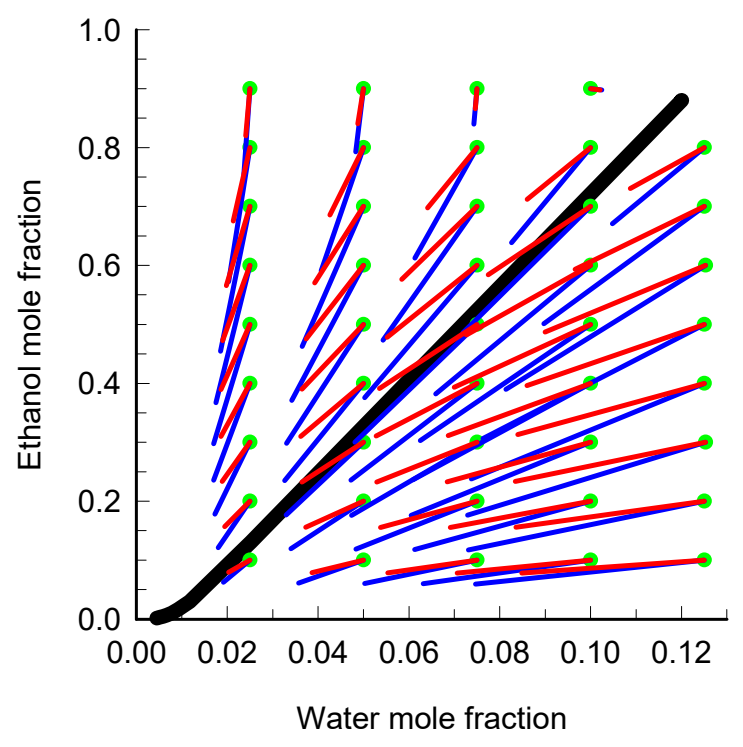

Fiq. S35

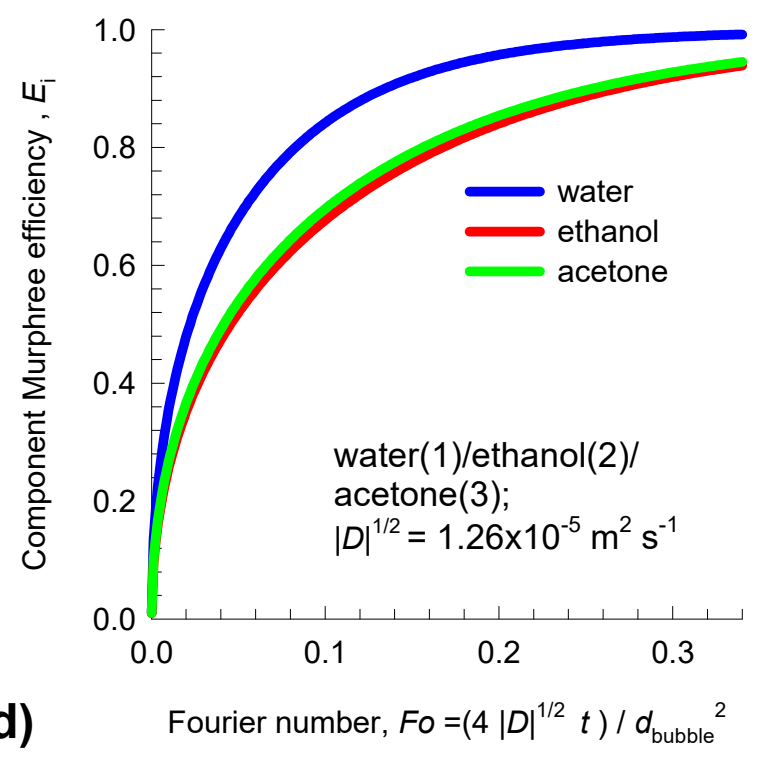

- Residue curve

$\longrightarrow$ Distillation Boundary

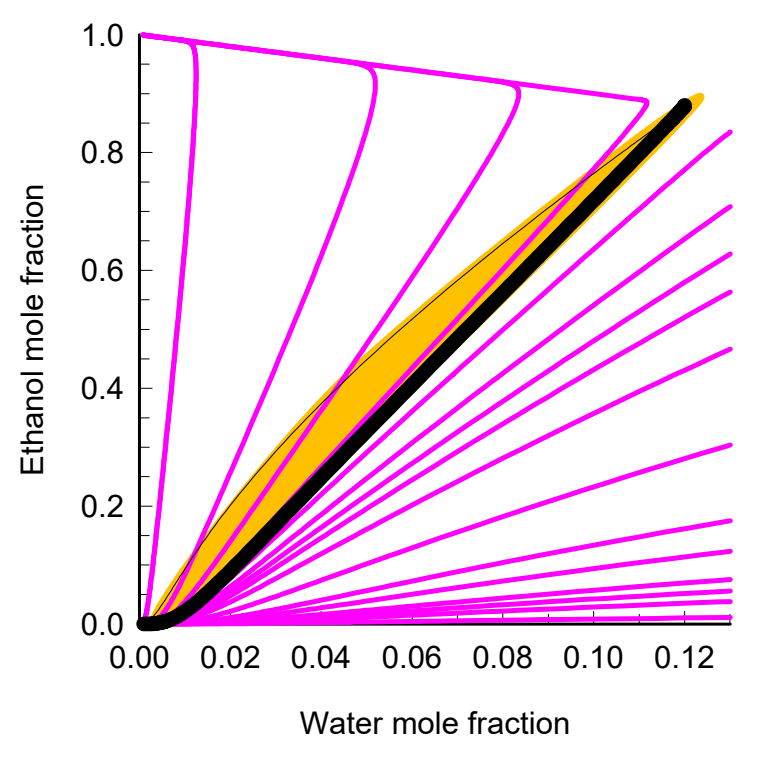




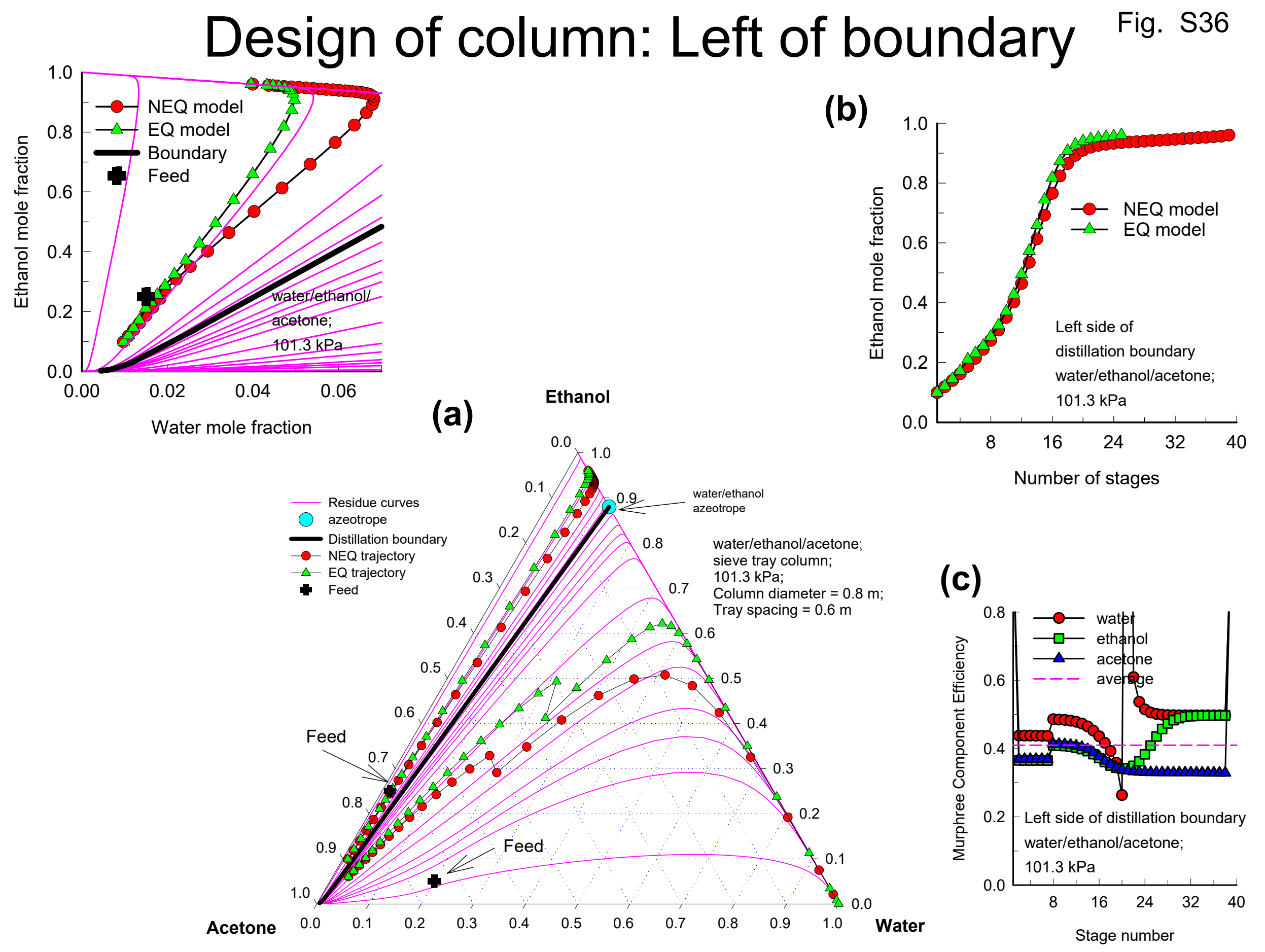




\section{Design of column: Right of boundary ${ }^{\text {Fig. }} 537$}

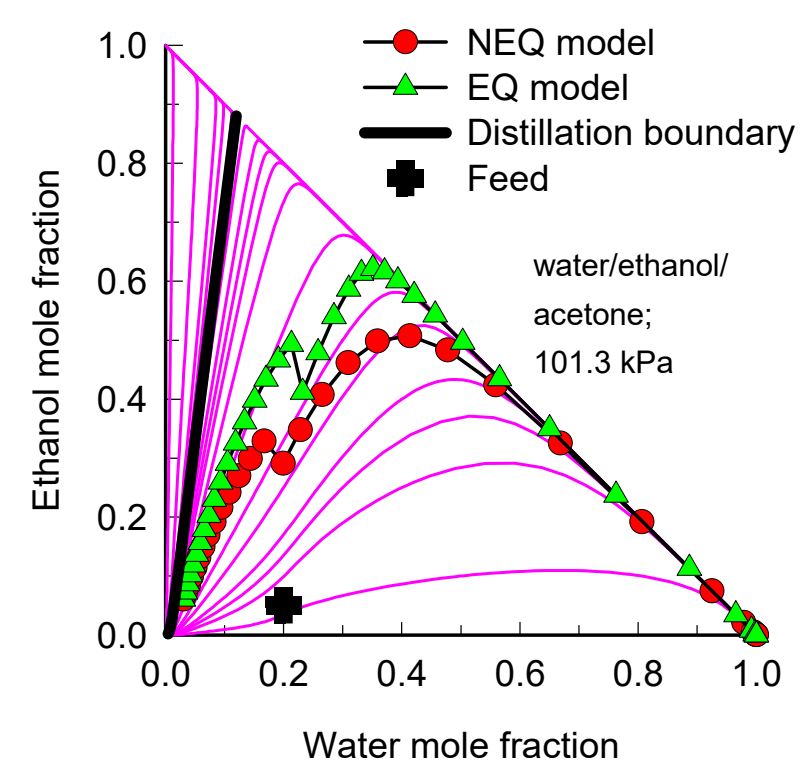

(a)

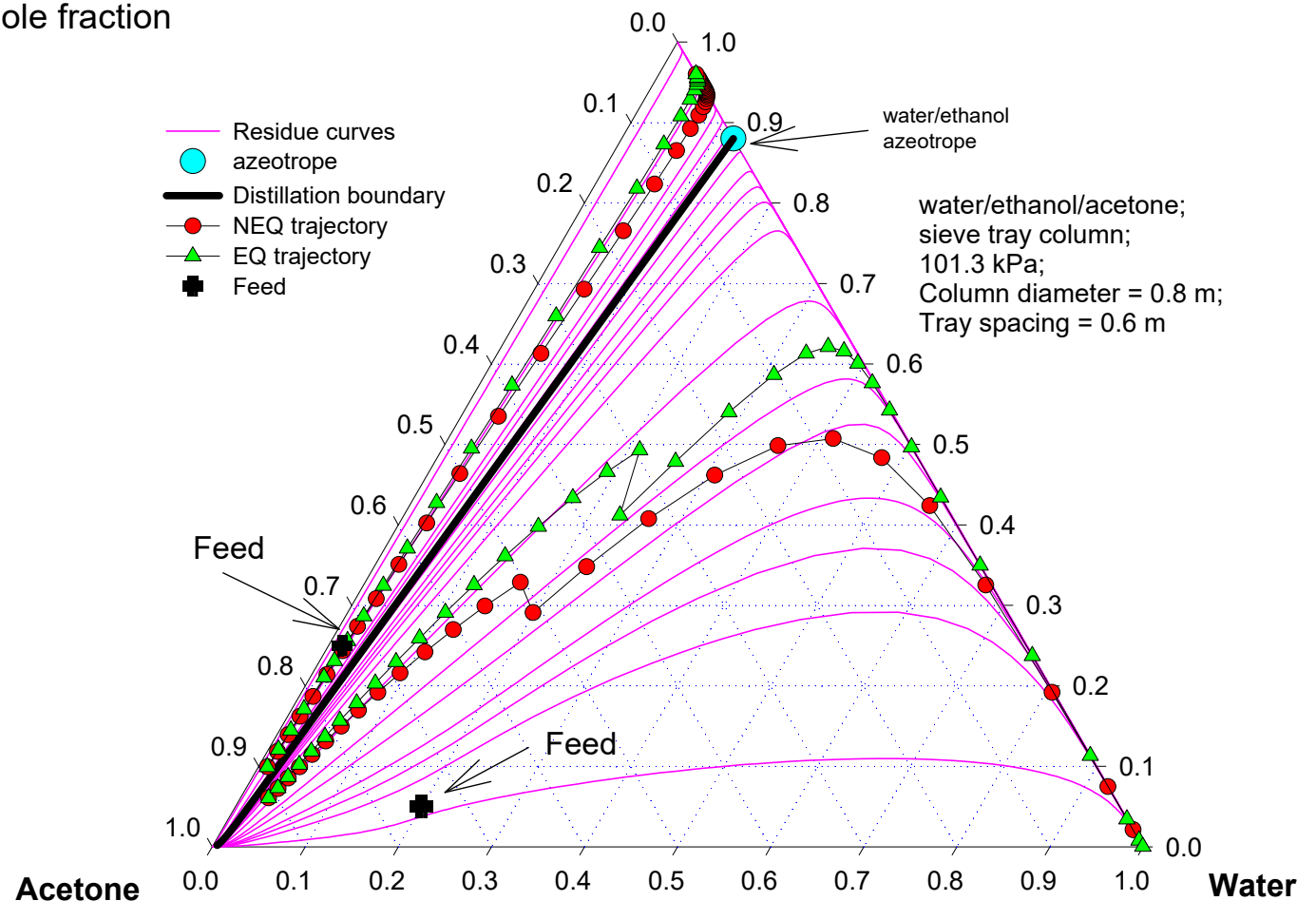

(b)

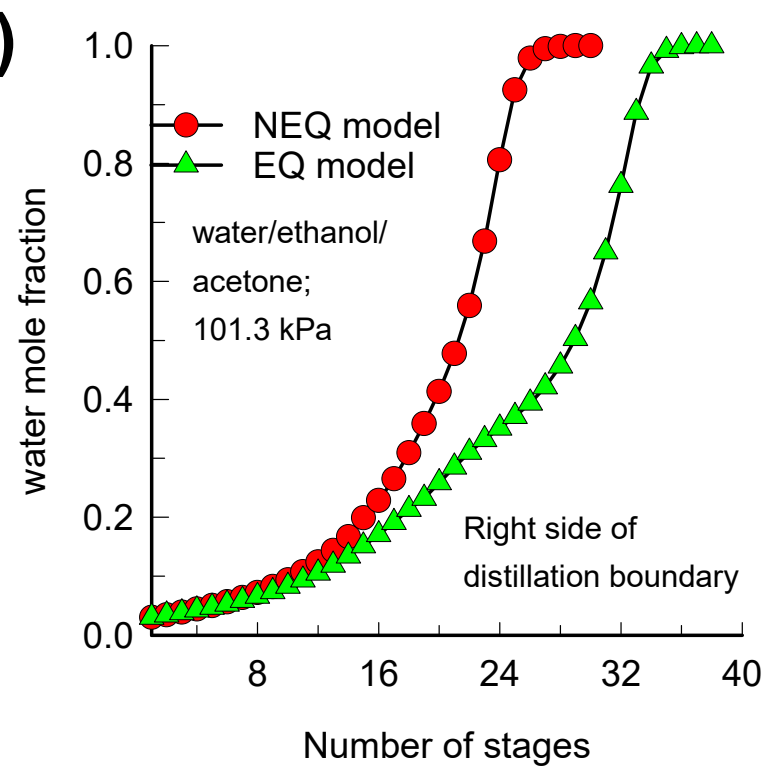

(c)

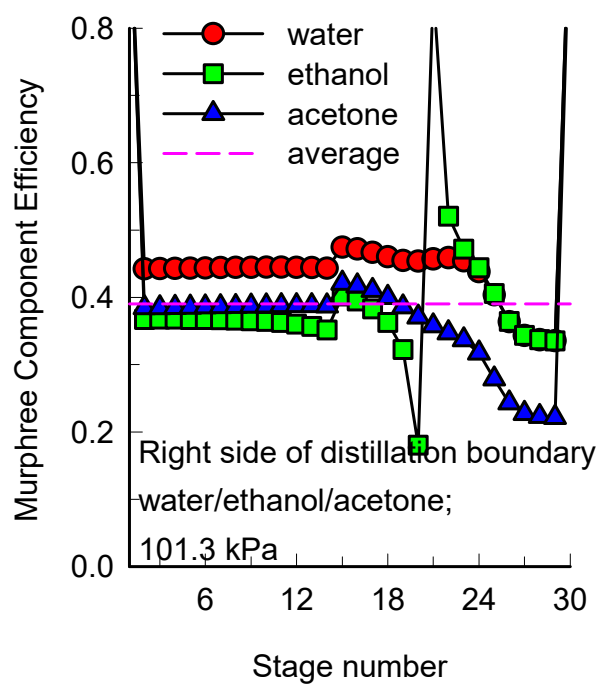


(a)

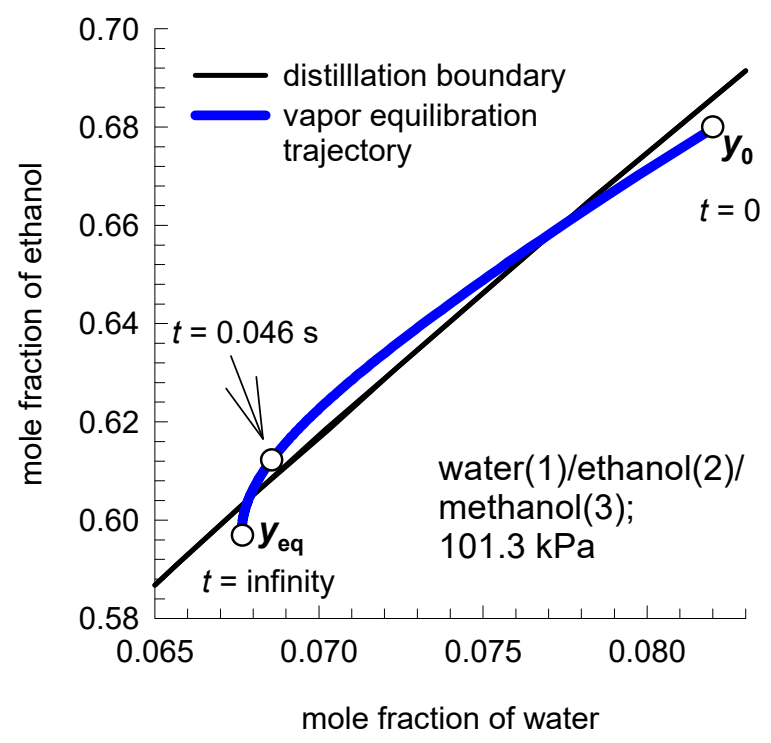

(c)

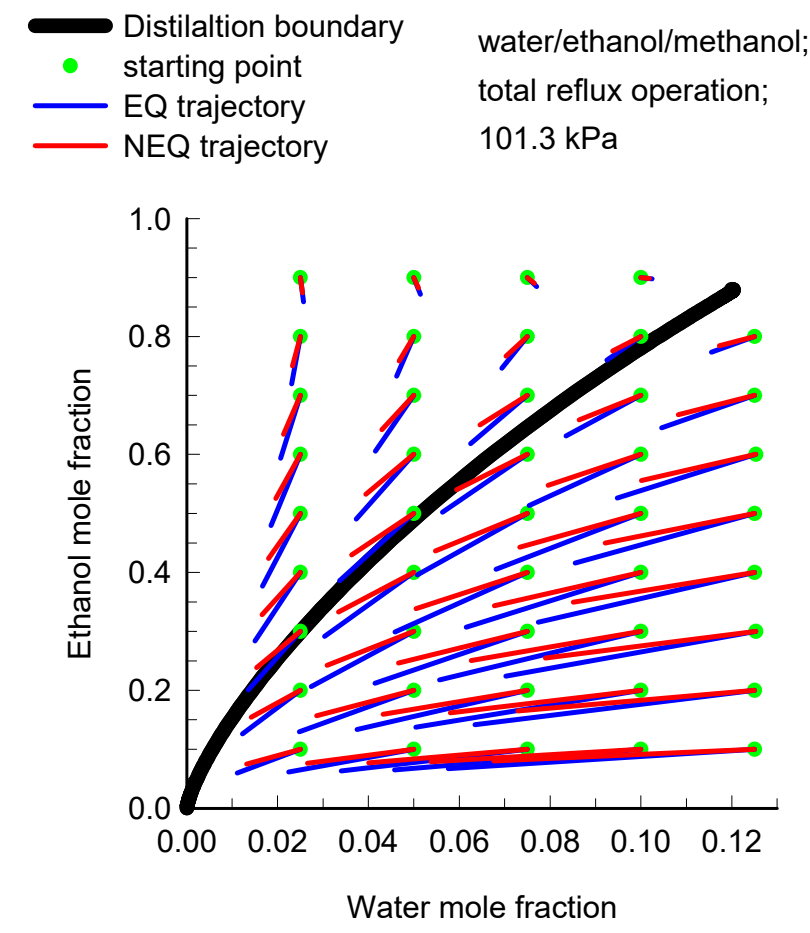

Fig. S38

(b)

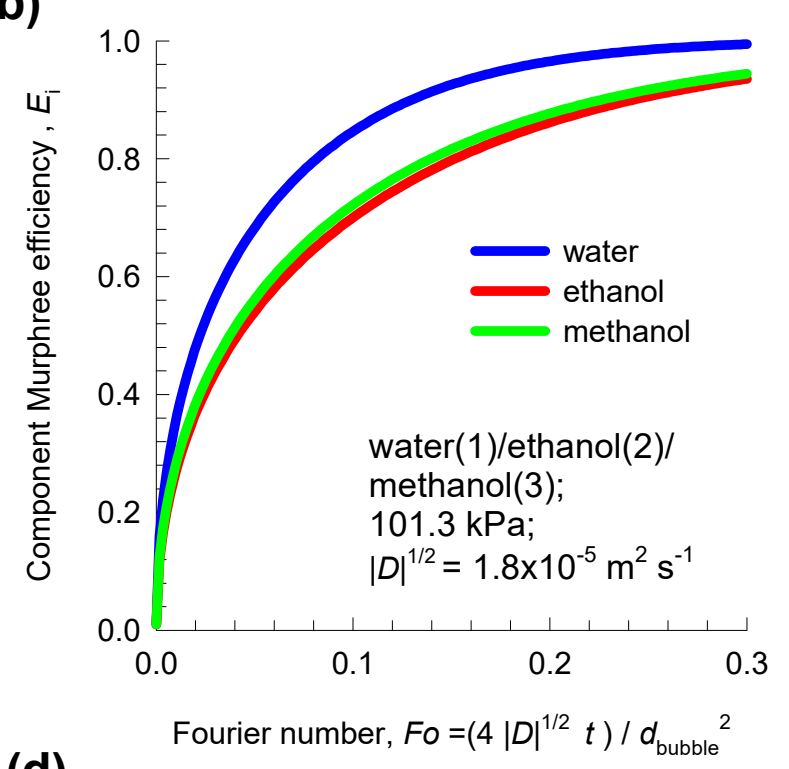

(d)

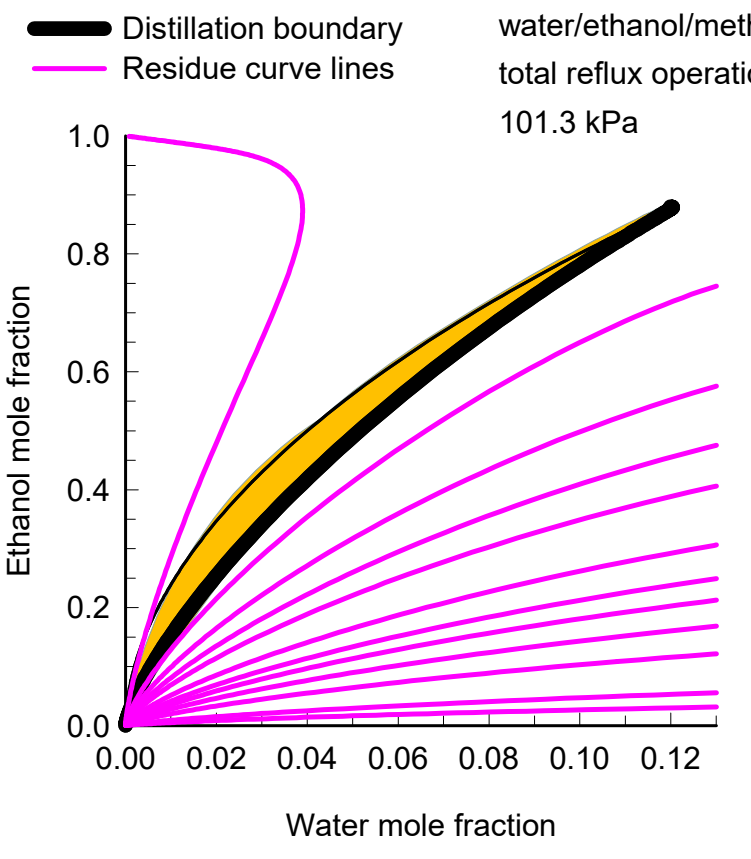




\section{Water/Ethanol/Methylacetate Distillation ${ }^{\text {fig. }}$ \$39}

(a)

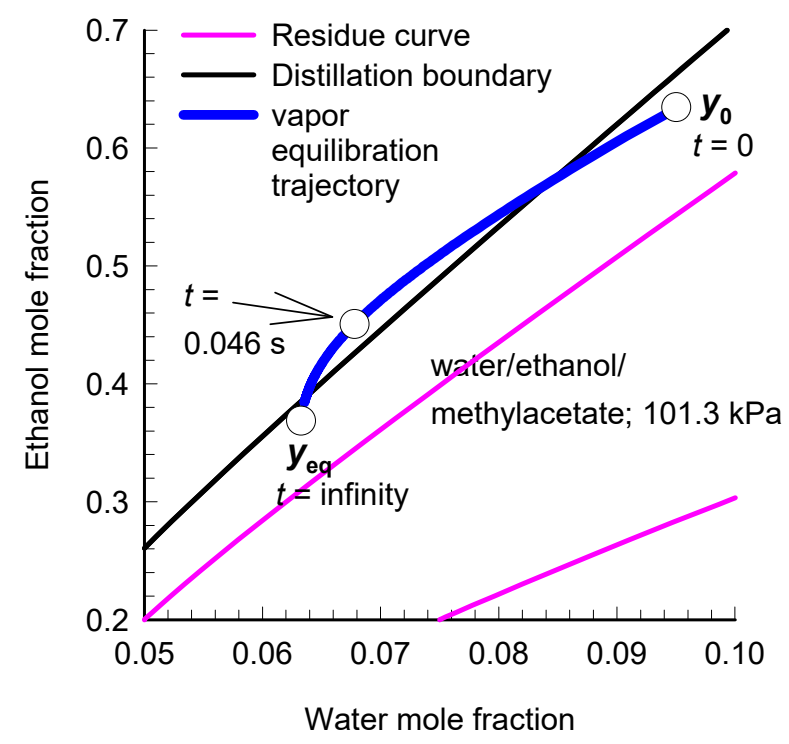

(c)

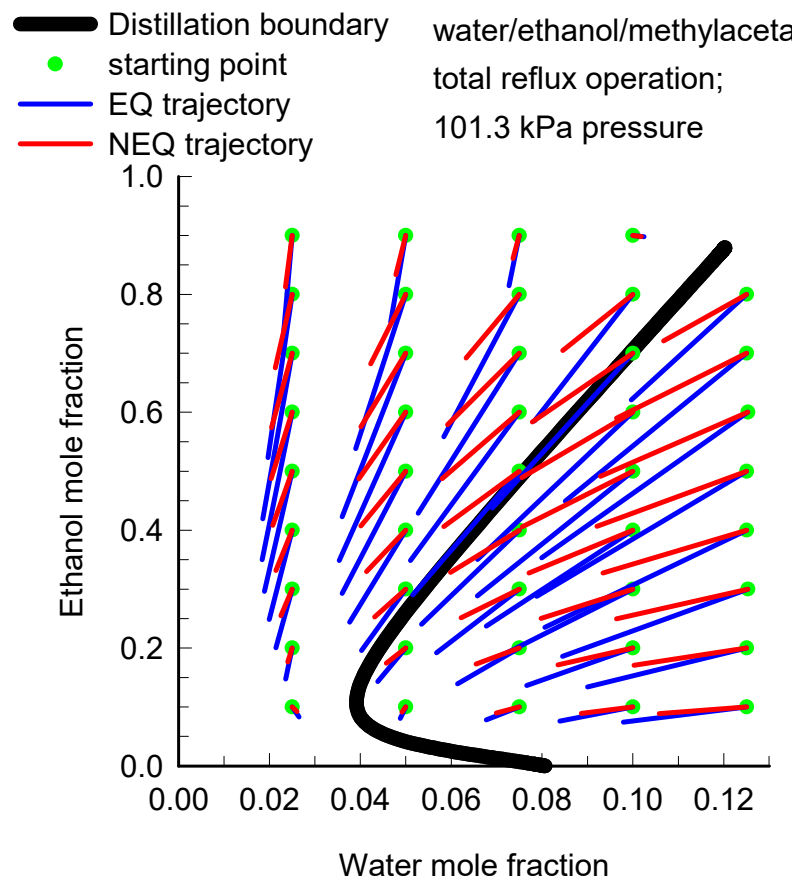

(b)

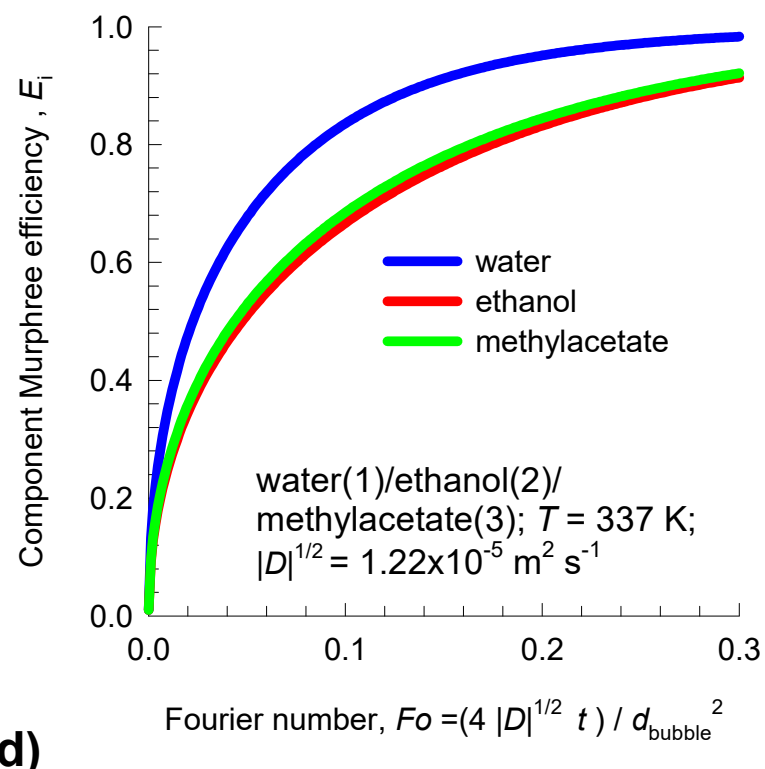

(d)

Distillation boundary
Residue curve

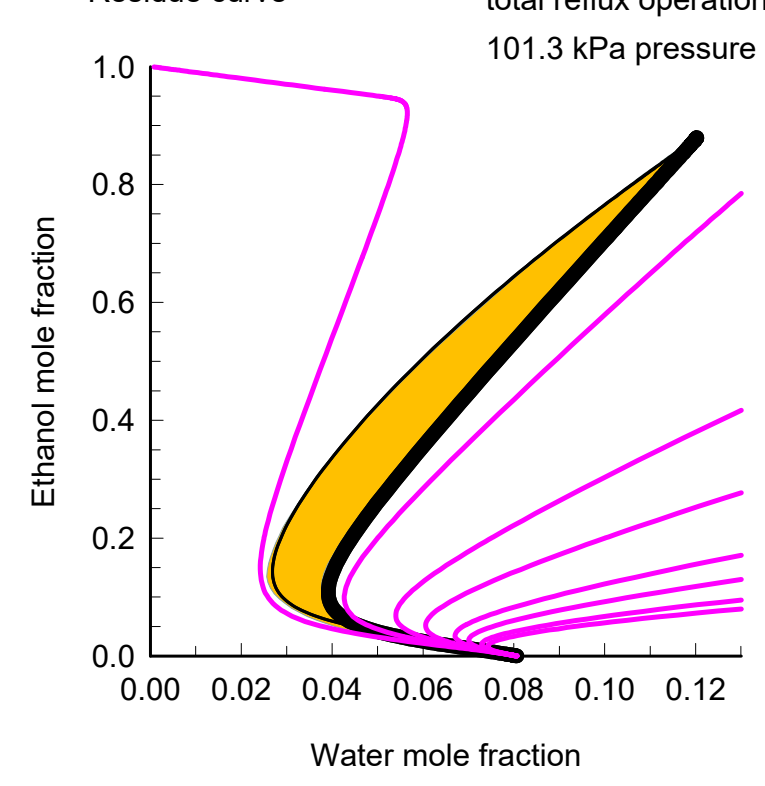

water/ethanol/methylacetate; total reflux operation; 101.3 kPa pressure

.




\section{Water/Acetone/Toluene Distillation}

Fig. S40
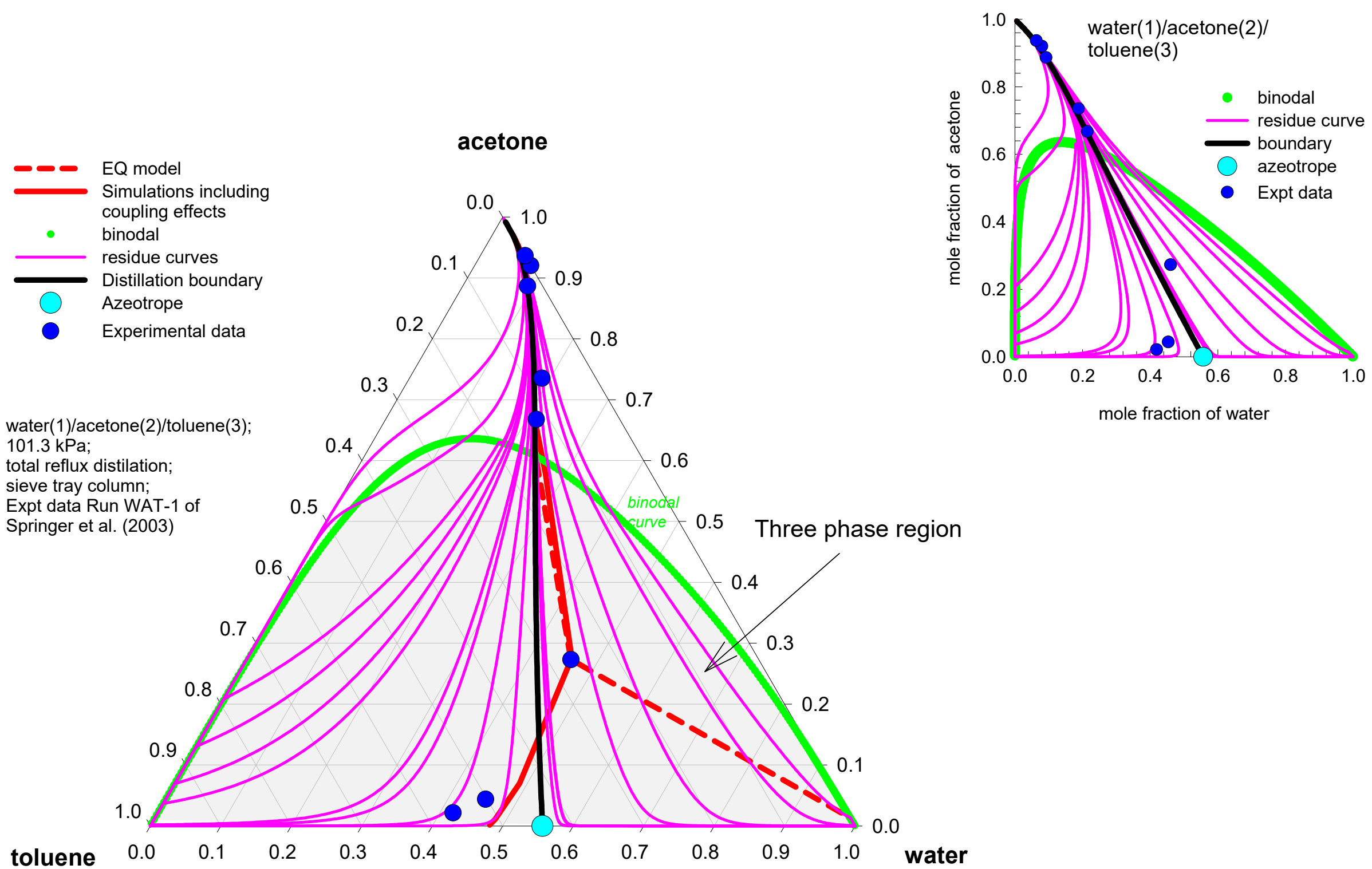


\section{Water/Acetone/Toluene Distillation}
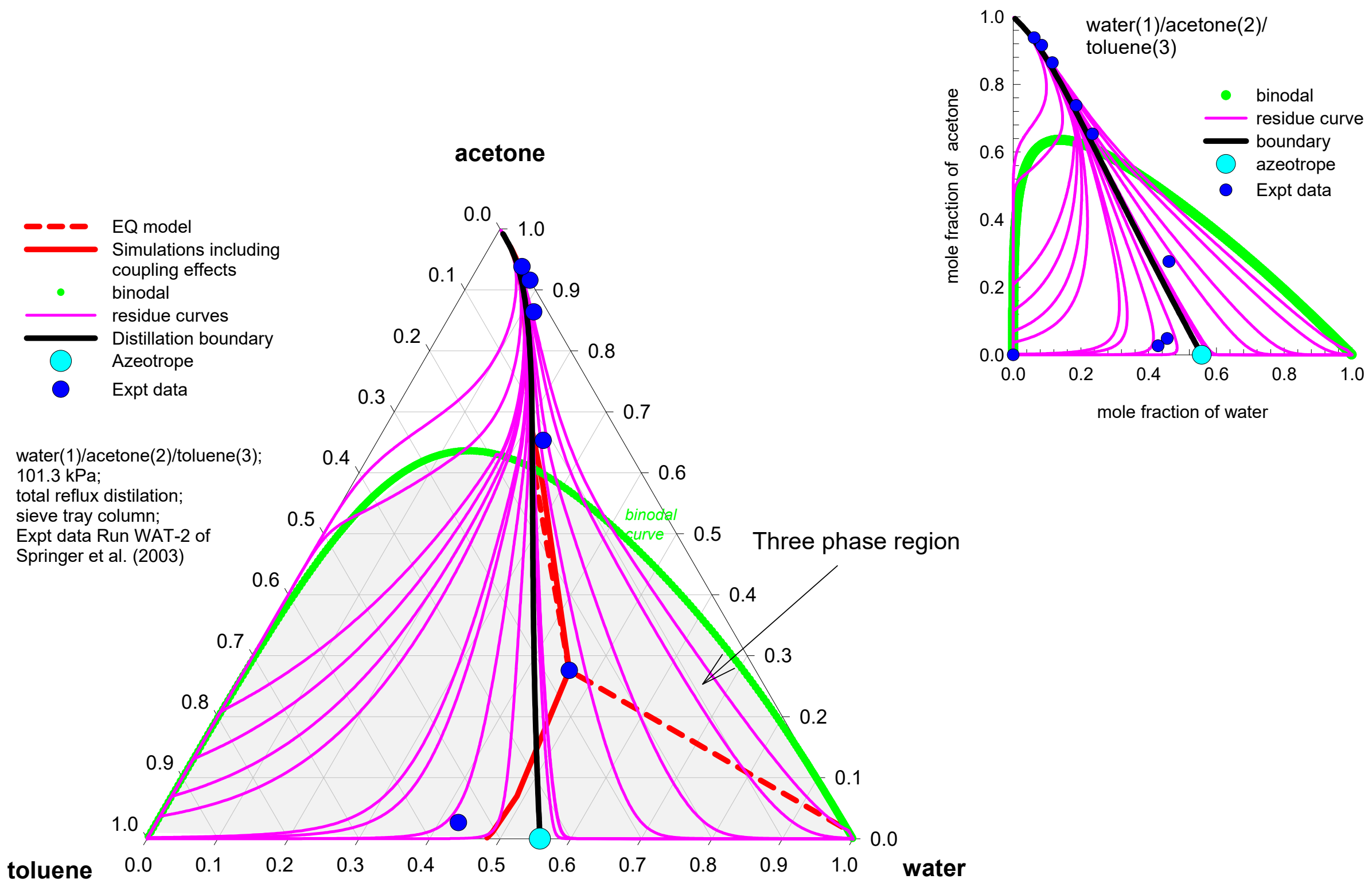


\section{Water/Ethanol/Cyclohexane Distillation ${ }^{\text {Fig. }}$ S42}

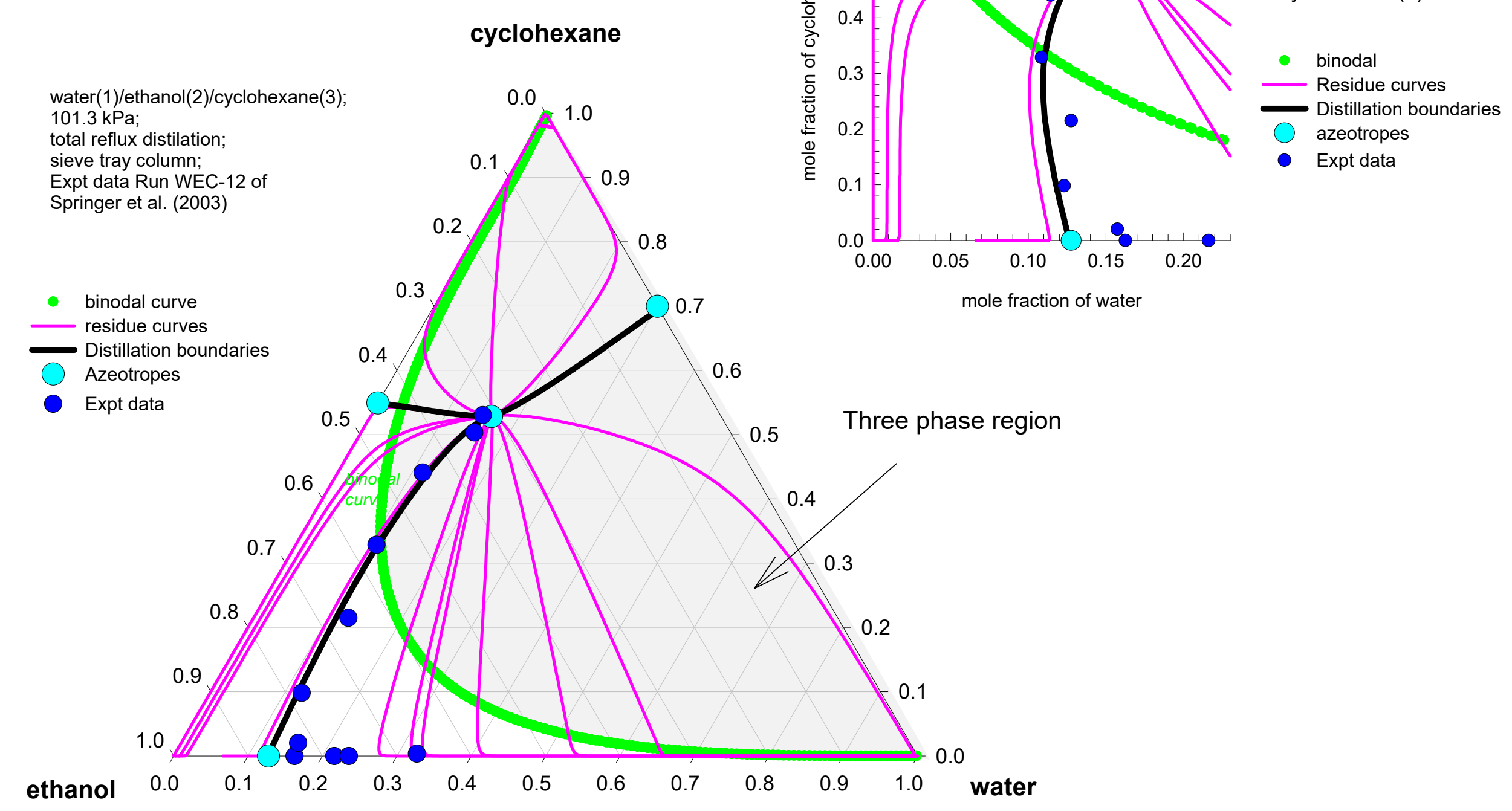




\section{Water/Ethanol/Cyclohexane Distillation ${ }^{\text {Fig. }}$ S43}

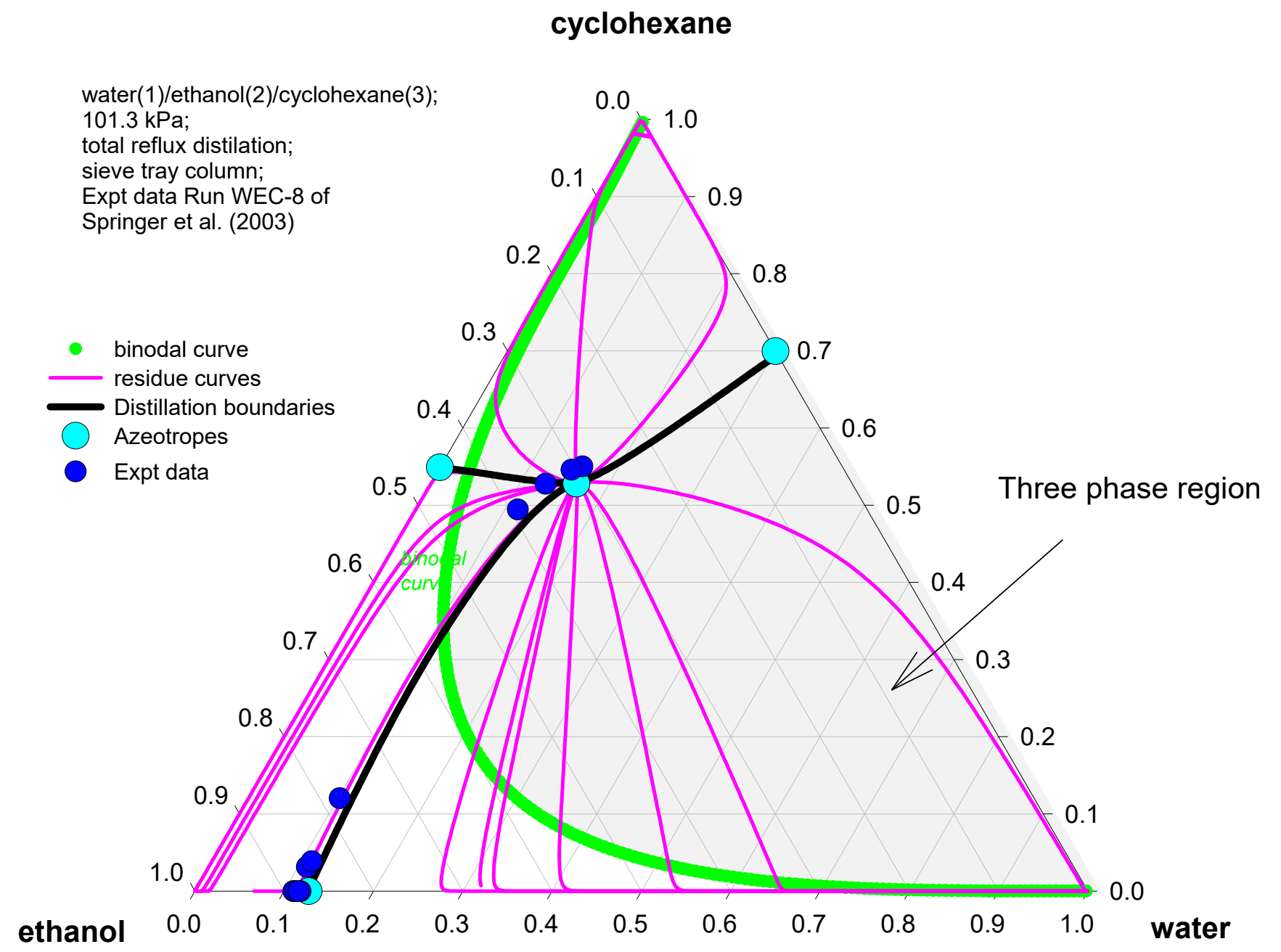




\section{Mass transfer resistances:Heterogeneouig. ${ }^{\text {Fi4 }}$ Azeotropic Distillation}

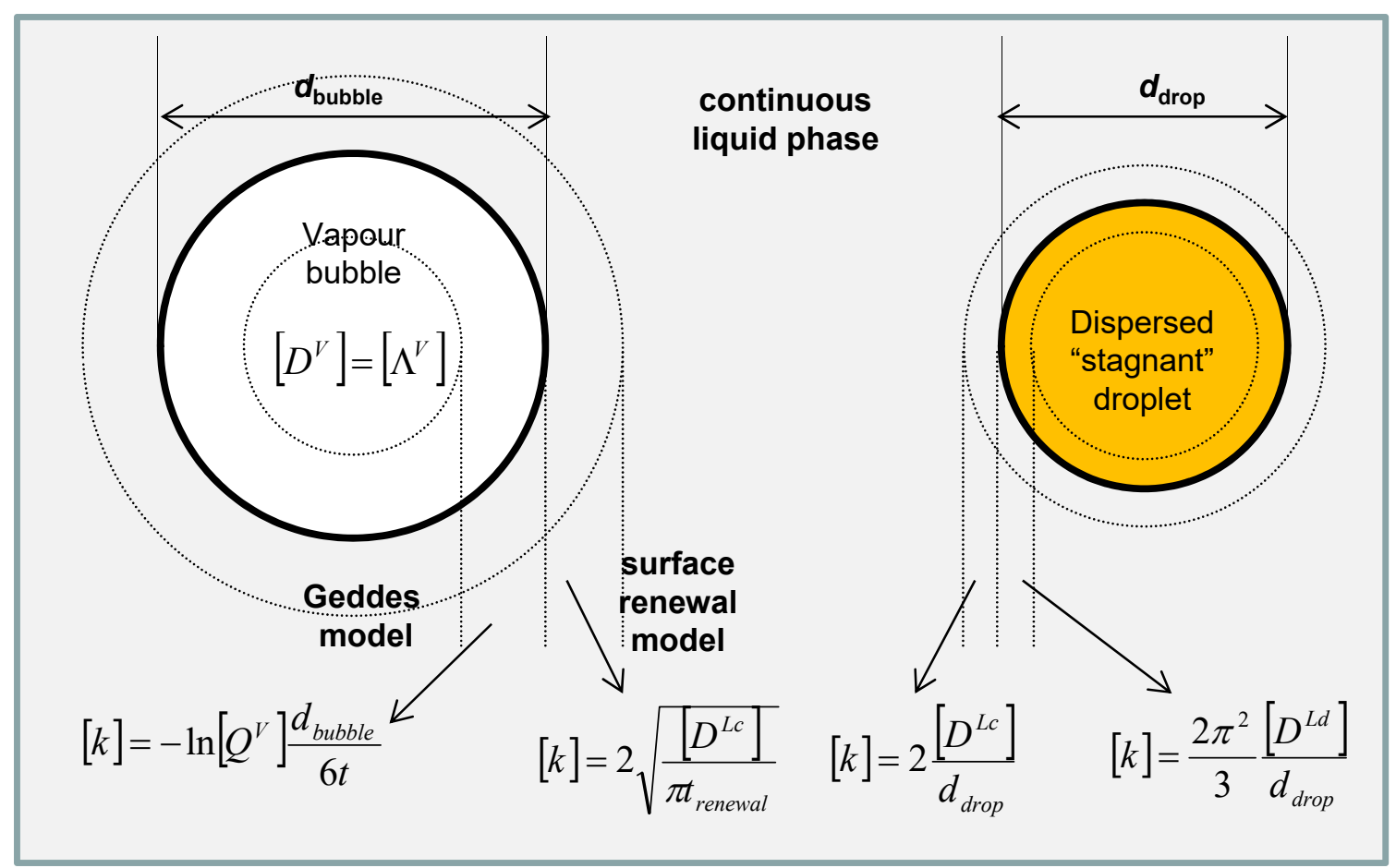




\section{Diffusional Distillation}

Fig. S45

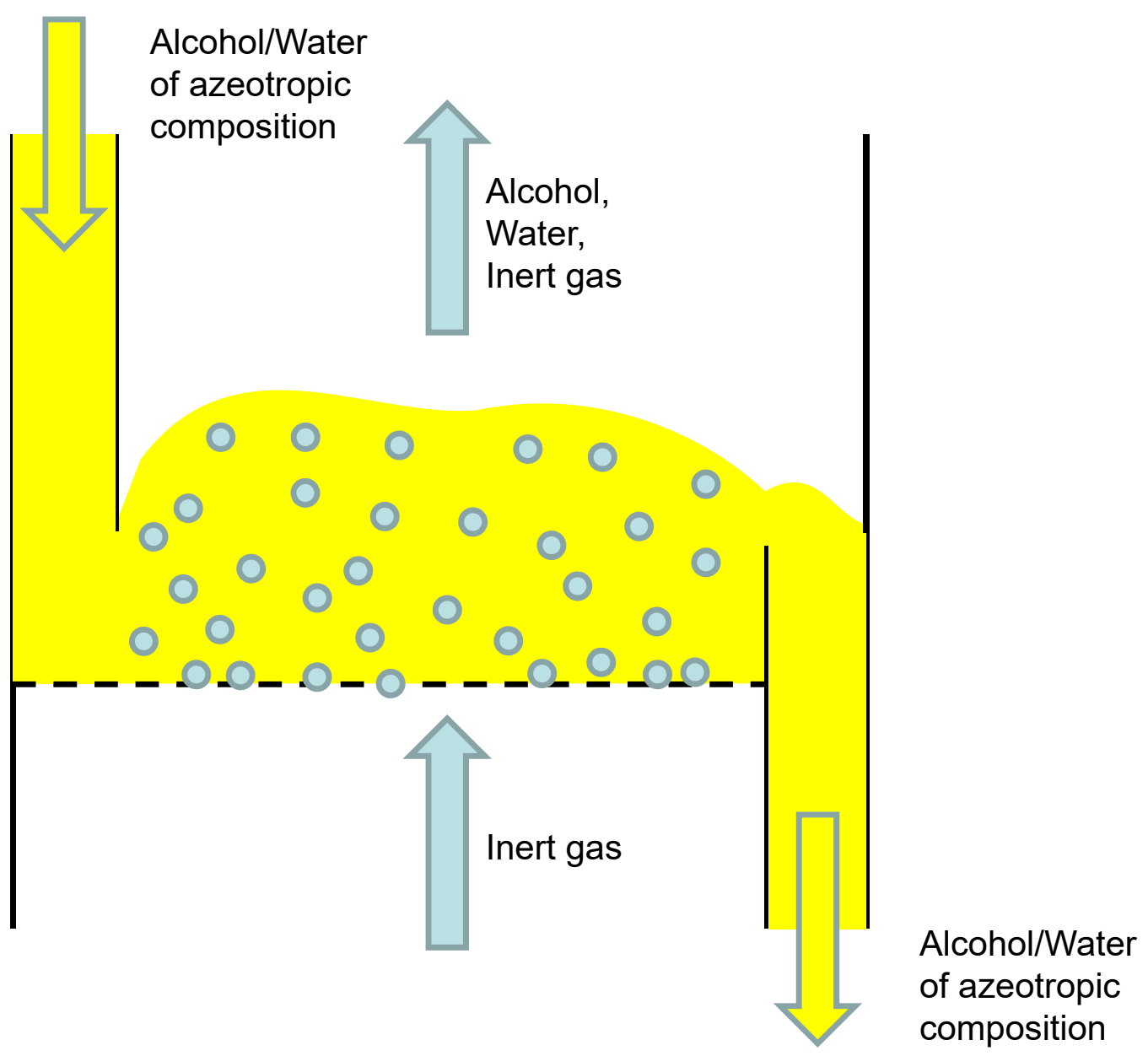




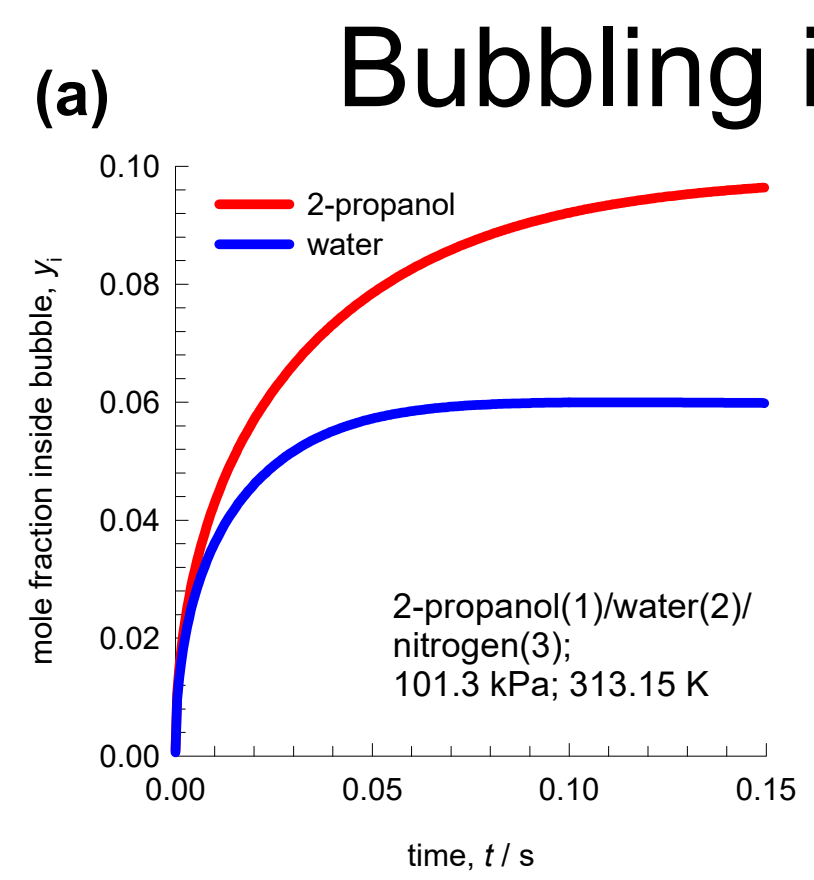

\section{(c)}

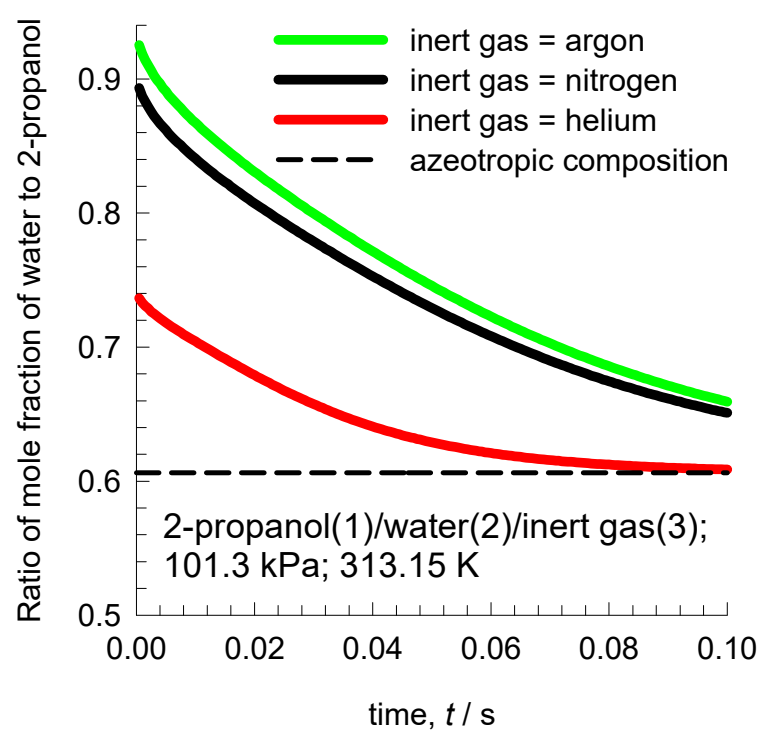

(b)

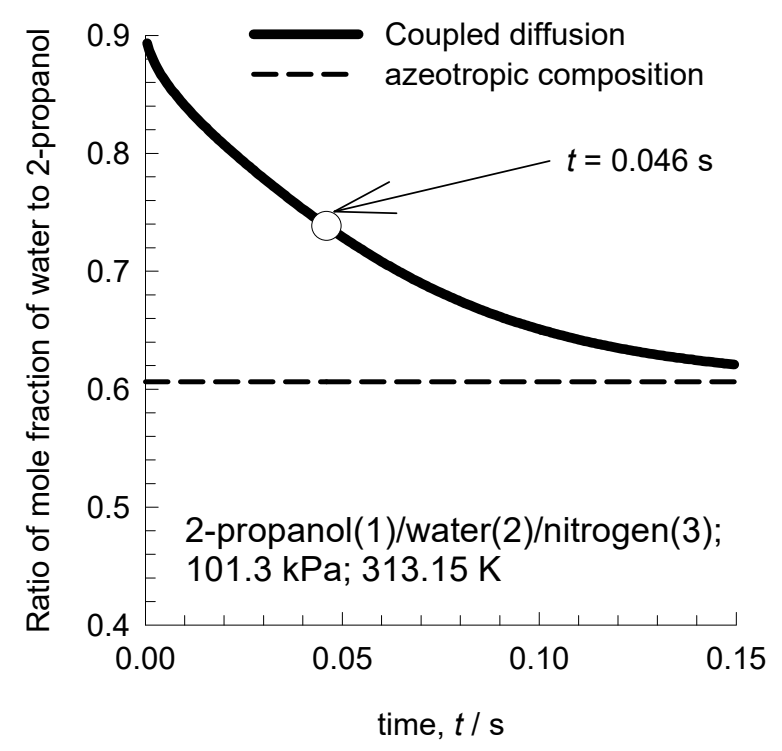

(d)

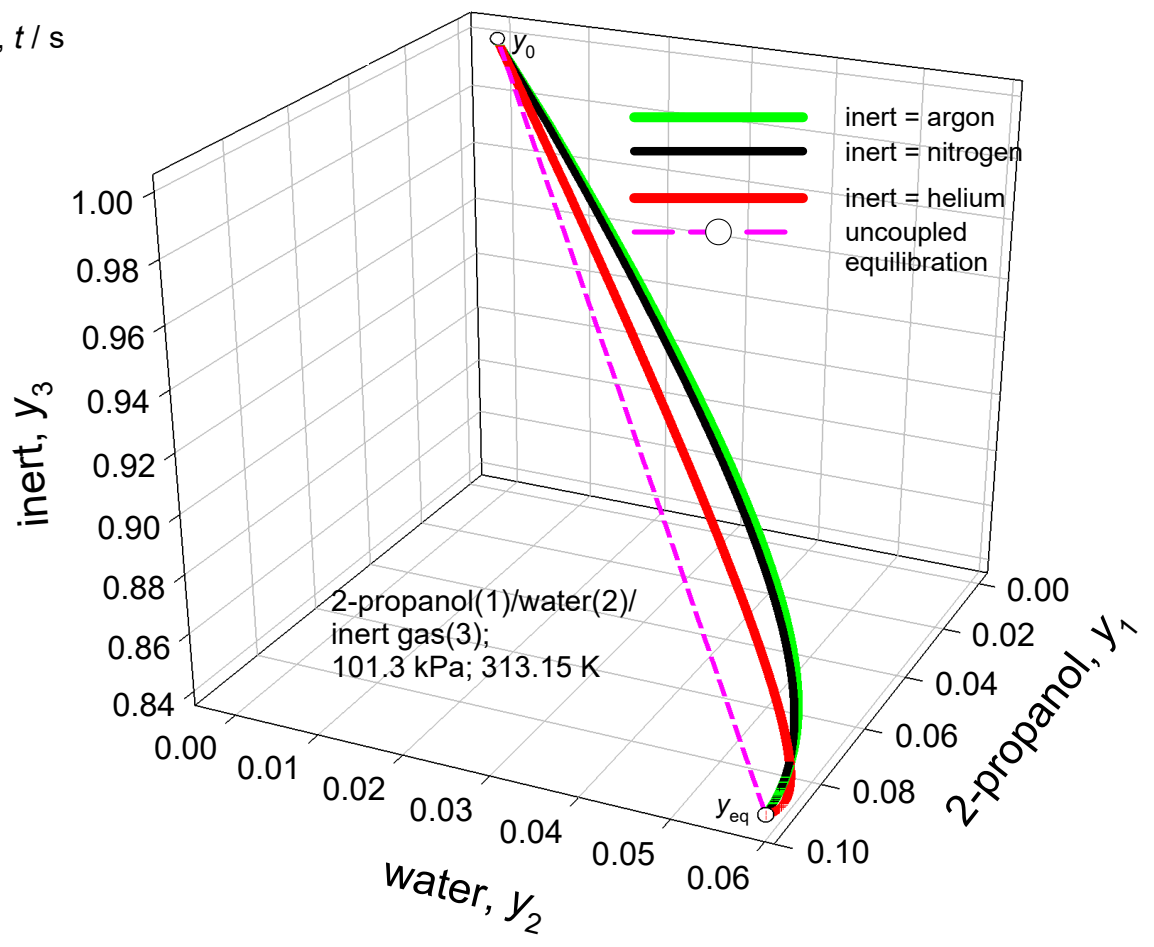




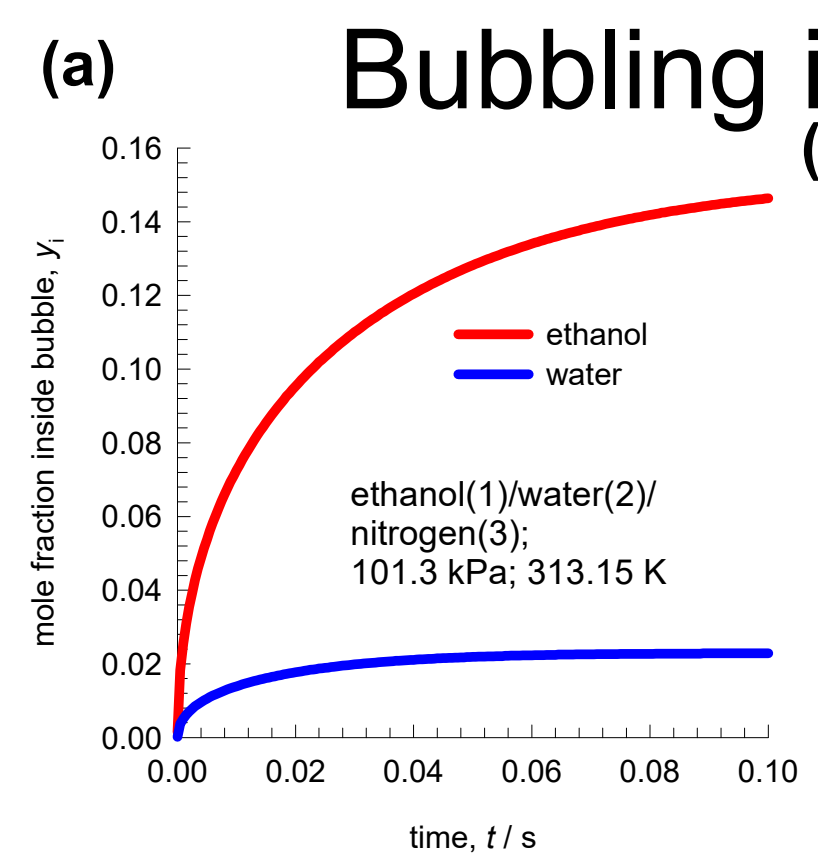

inert gas to break azeotrope $^{\text {Fig. } 547}$

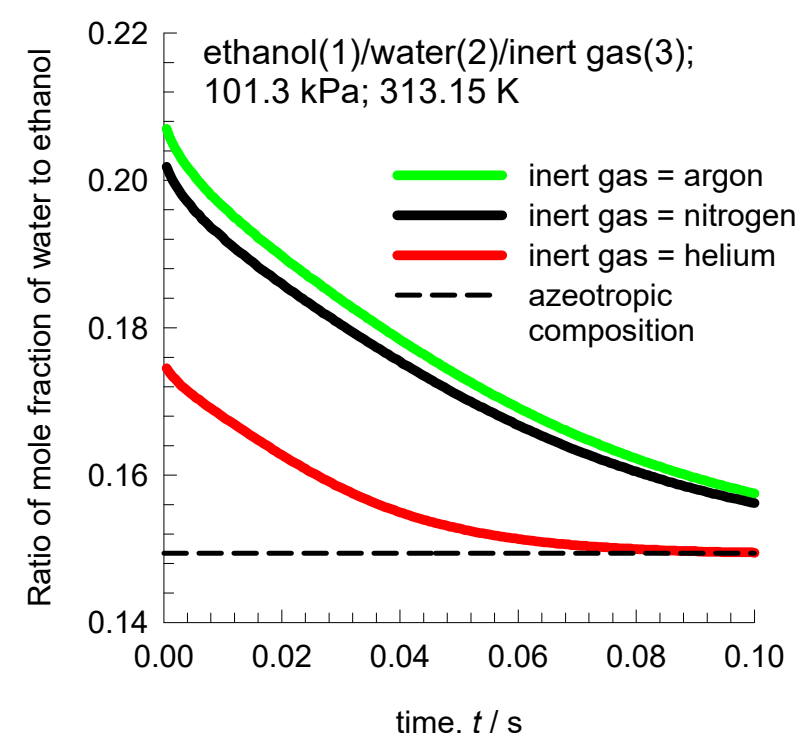

(b)

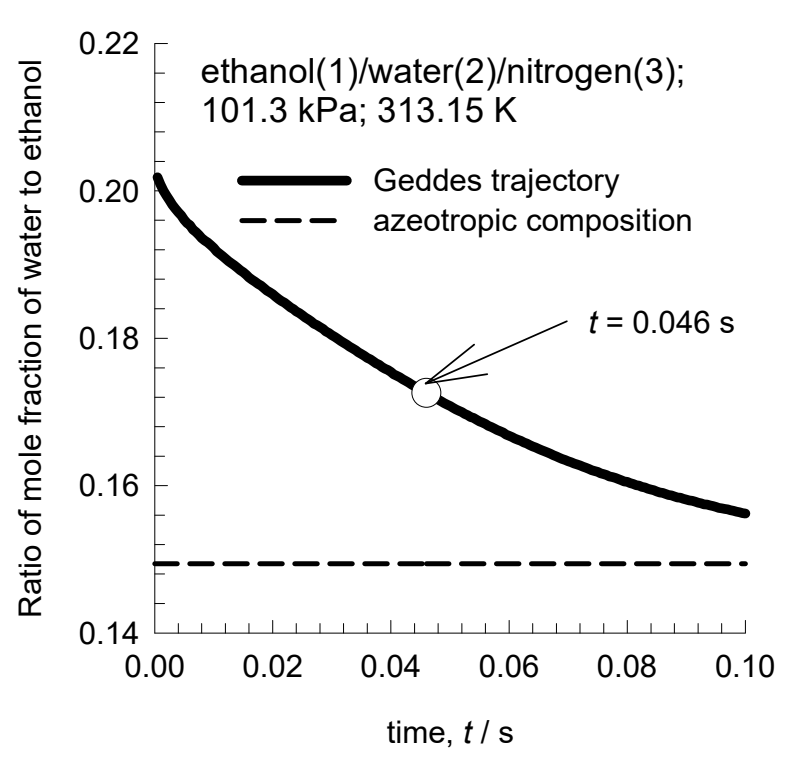

(d)

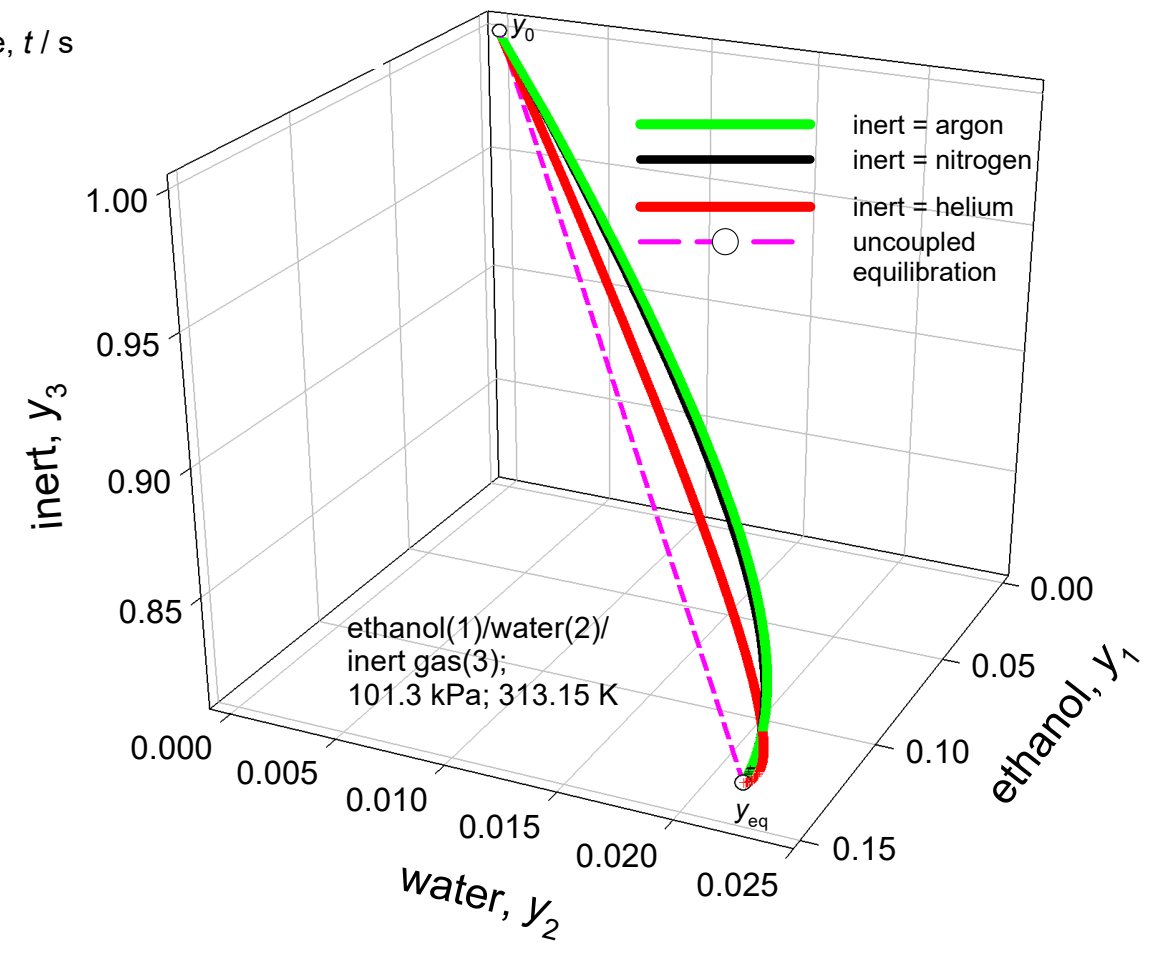



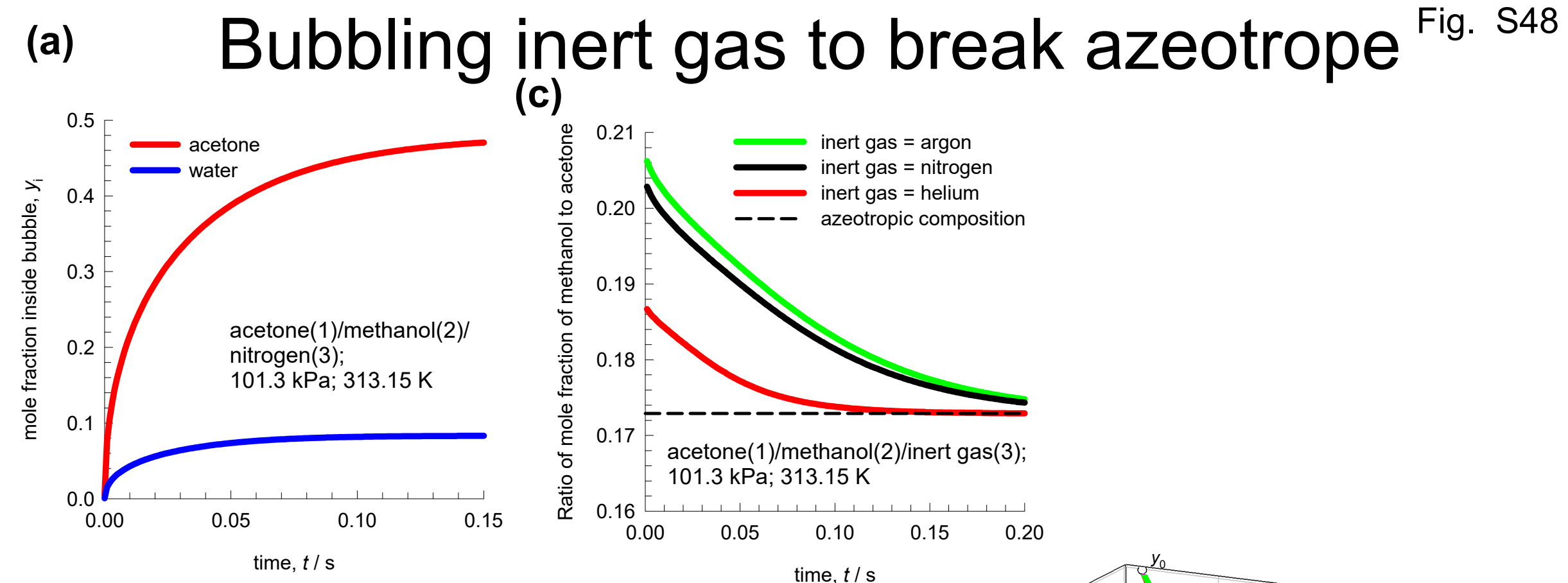
(c)

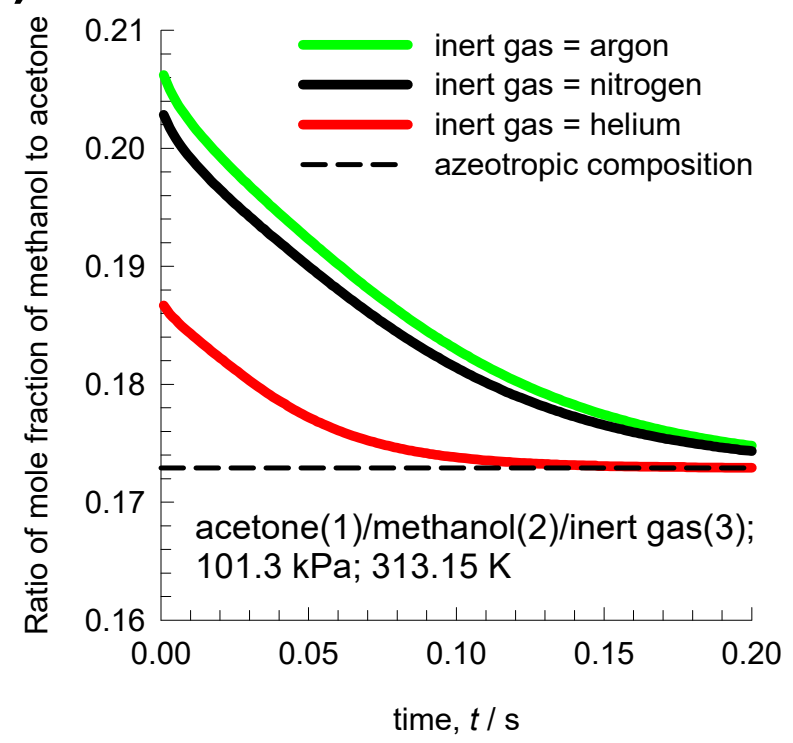

(b)

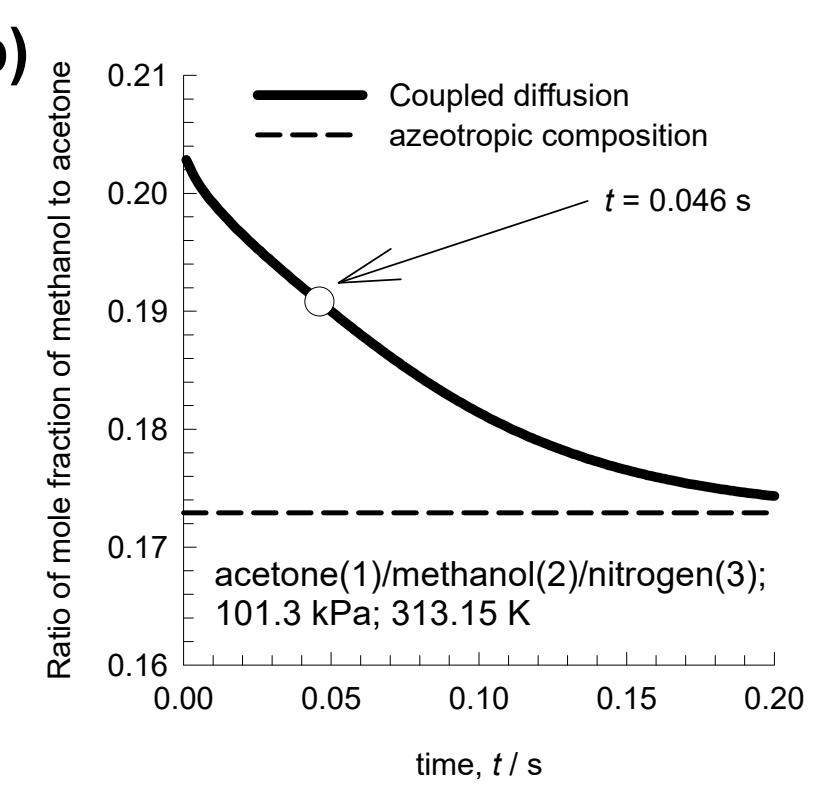

(d)

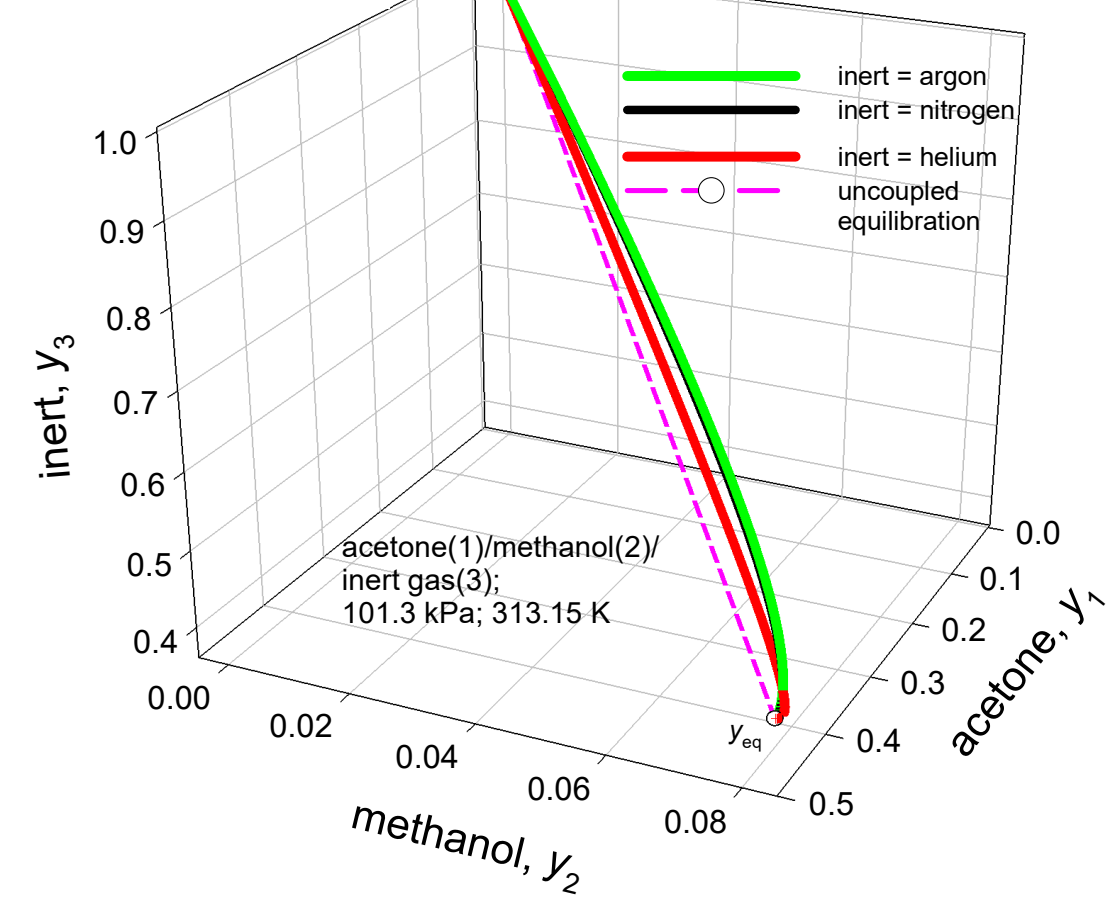

